\title{
Observationally driven 3D MHD model of the solar corona above a magnetically active region
}

\author{
Dissertation \\ zur Erlangung des mathematisch-naturwissenschaftlichen \\ Doktorgrades \\ "Doctor rerum naturalium" (Dr. rer. nat.) \\ der Georg-August-Universität Göttingen \\ im Promotionsprogramm PROPHYS \\ der Georg-August University School of Science (GAUSS)
}

\author{
vorgelegt von \\ Philippe-André Bourdin \\ aus Friedberg (Hessen)
}

Göttingen, 2013 
Betreuungsausschuss

Prof. Dr. Jens Niemeyer

Institut für Astrophysik, Georg-August-Universität Göttingen

Prof. Dr. Wolfgang Glatzel

Institut für Astrophysik, Georg-August-Universität Göttingen

Prof. Dr. Hardi Peter

Max-Planck-Institut für Sonnensystemforschung, Katlenburg-Lindau

Mitglieder der Prüfungskommission

Referent: Prof. Dr. Hardi Peter

Max-Planck-Institut für Sonnensystemforschung, Katlenburg-Lindau

Koreferent: Prof. Dr. Wolfgang Glatzel

Institut für Astrophysik, Georg-August-Universität Göttingen

Weitere Mitglieder der Prüfungskommission:

Prof. Dr. Manfred Schüssler

Max-Planck-Institut für Sonnensystemforschung, Katlenburg-Lindau

Prof. Dr. Laurent Gizon

Institut für Astrophysik, Georg-August-Universität Göttingen

Prof. Dr. Ansgar Reiners

Institut für Astrophysik, Georg-August-Universität Göttingen

Prof. Dr. Uli Christensen

Max-Planck-Institut für Sonnensystemforschung, Katlenburg-Lindau

Tag der mündlichen Prüfung: 26.09.2013 


\section{Bibliografische Information der Deutschen Nationalbibliothek}

Die Deutsche Nationalbibliothek verzeichnet diese

Publikation in der Deutschen Nationalbibliografie;

detaillierte bibliografische Daten sind im

Internet über http://www.DNB.de/katalog abrufbar.

\section{Umschlagseite:}

Synthetisierte Emission von extrem-UV- sowie Röntgenlicht eines 3D-MHD-Modells der Sonnenkorona über einer magnetisch aktiven Region.

\section{Cover page:}

Synthesized emission of extreme-UV light and X-rays of a 3D MHD model of the Solar corona above a magnetically active region.

ISBN 978-3-944072-03-6

uni-edition $\mathrm{GmbH} 2014$

http://www.uni-edition.de/

(c) Philippe-André Bourdin

http://www.Bourdin.ch/Philippe/

This work is distributed under a

Creative Commons Attribution 3.0 License

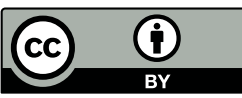

Printed in Germany 
Mathematik ohne einen Anwendungsbezug stellt nur ein Werkzeug zur Abstraktion logischer Zusammenhönge dor.

Physik ohne eine philosophische Reflektierung kann nur ein Werkzeug zur mathematischen Beschreibung und Voraussage von Naturphönomenen sein.

Ein tieferes Naturverstöndnis erfordert daher den erkenntnistheoretischen Diskurs in der Physik.

"Nichts existiert nicht."

(Philosophische Erkenntnis) 


\section{Contents}

Zusammenfassung

Summary

1 Introduction

2 Technical introduction 2

2.1 Treatment of space plasmas ............... 21

2.1.1 Magnetohydrodynamics . . . . . . . . . . . . 21

2.1.2 Quasi-neutrality ................ 21

2.1.3 Plasma beta ................. . . . 22

2.1 .4 The equations ................ 23

2.2 The code .......................... 24

2.3 Coronal model setup . . . . . . . . . . . . . . 26 26

2.3.1 Basic idea . . . . . . . . . . . . . . . 26

2.3.2 Model parameters ... . . . . . . . . . . . 26

2.3.3 Initial condition . . . . . . . . . . . . . . . 27

2.3.3.1 Stratified atmosphere . . . . . . . . . . 27

2.3.3.2 Initial magnetic field . . . . . . . . . . 28

2.3.3.3 Switching on ............. 28

2.3.3.4 Self-consistent model . . . . . . . . . . . . 29

2.3.4 Boundary conditions ... . . . . . . . . . . 30

2.3.4.1 Photospheric magnetic field . . . . . . . . 30

3 Observationally driven model of the corona above an AR 33

3.1 Motivation ...................... 34

3.2 Model strategy . . . . . . . . . . . . . . 35

3.3 MHD model and alignment with observations ......... 37

3.3.1 Coronal model . . . . . . . . . . . . . . . . . . 37

3.3.2 Lower boundary condition from observations . . . . . . . 39 
3.3.3 Alignment between observations and simulation ..... 39

3.4 Hot loops in the core of the active region . . . . . . . . . . 40

3.5 STEREO 3D reconstruction .................... 42

3.6 Conclusions . . . . . . . . . . . . . . . . . . . 44

4 Coronal energy input and dissipation 45

4.1 Motivation . . . . . . . . . . . . . . . . . . . 45

4.2 Energy input viewed at large . . . . . . . . . . . 45

4.2.1 Overview of whole box and subvolumes . . . . . . . . 45

4.2.1.1 Contribution to the internal energy ...... 45

4.2.1.2 Contribution to the temperature ........ 47

4.2.1.3 Energy introduced into the model . . . . . . . 49

4.3 Statistical analyses of coronal field lines . . . . . . . . . . 51 51

4.3.1 Coronal energy conversion . . . . . . . . . . . 52 52

4.3.1.1 Poynting flux as energy source . . . . . . . 52 52

4.3.1.2 Ohmic heating integrated along field lines . . 5 54

4.3.1.3 Mean Ohmic heating . . . . . . . . . . 56

4.3.1.4 Relation to magnetic field strength ....... 57

4.3.2 Scaling laws for coronal loop properties . . . . . . . . . . 60

4.3.3 Scaling laws in 3D model .............. 61

4.3.3.1 Heating and loop length .......... 61 61

4.3.3.2 Loop temperature . . . . . . . . . . . 62

4.3.3.3 Loop density . . . . . . . . . . . . . 65

4.3.4 Parameterization of Ohmic heating . . . . . . . . 66 66

4.3.4.1 van Ballegooijen scaling law ......... 66

4.3.4.2 Rappazzo scaling law . . . . . . . . . . . 68

4.3.4.3 Fitting a new scaling law ........ . . 70

4.4 Conclusions . . . . . . . . . . . . . . . . . 72

5 The transition region Doppler shifts contradiction 73

5.1 Motivation . . . . . . . . . . . . . . . . . . 73

5.2 Doppler-shift statistics . . . . . . . . . . . . . . 73

5.2.1 Synthetic spectra from MHD model . . . . . . . . . . . 74

5.2.2 Spectral properties compared to observations . . . . . . 74

5.2.2.1 Estimating an effective Doppler shift. . . . . . 76

5.2.2.2 Properties of selected synthetic spectra . . . . 77

5.2.2.3 Comparing to observations . . . . . . . . . 78

5.3 Heating events as driver of Doppler shifts . . . . . . . . . . . 81

5.3.1 Pressure gradient analysis ............. 81 
5.3.2 Upwards pressure acceleration . . . . . . . . . . . 82

5.3.3 Downwards pressure acceleration ........... 82

5.3.4 Location of maximum acceleration . . . . . . . . . . 83

5.4 Investigation of prominent individual structures . . . . . . . 86

5.4.1 Properties of a coronal loop . . . . . . . . . . . . 86

5.4.1.1 Loop SL 1 investigated . . . . . . . . . . 86

5.4.1.2 Loop SL 2 investigated .......... . 89

5.4.1.3 Siphon flow explained .......... 89

5.4.2 Total spetcra for an individual loop . . . . . . . . . . 90 90

5.4.2.1 Footpoint emission of a loop . . . . . . . 9 90

5.4.2.2 Integration along a loop ......... 91

5.4.2.3 Integration of a vertical cut ....... . . 92

5.5 Conclusions . . . . . . . . . . . . . . . . . . . . . 94

6 Discussion of the results 95

6.1 Modeling of coronal features . . . . . . . . . . . . . 95

6.2 Abstraction of the coronal heating . . . . . . . . . . 95

6.3 TR Doppler shifts reproduced . . . . . . . . . . . . . . . . 96 96

6.4 Outlook ............................. 97

6.5 Executive summary .................... 97

Bibliography

A Appendix 107

A. 1 Serio correction factor unraveled . . . . . . . . . . . . . 107

Publications 111

Acknowledgements - Danksagung 113 



\section{Zusammenfassung}

Kontext: Die Sonnenkorona wird seit 1932 mit Koronographen beobachtet. Nur wenige Jahre später war klar, dass die Korona viel heißer ist als die sichtbare Sonnenoberfläche; seit dem ist der Mechanismus der koronalen Heizung ungeklärt. Viele Mechanismen wurden vorgeschlagen, die genügend Energie zur Basis der Korona liefern, es hat sich aber kein vollständig selbstkonsitentes Bild des Energietransports und der koronalen Dissipation etabliert.

Ziele: Wir möchten ein selbstkosistentes Modell aufstellen, welches Bewegungen auf der Sonnenoberfläche enthält, welche das Magnetfeld verbiegen und verflechten, wodurch in der Korona Ströme induziert und Ohm'sch dissipiert werden. Die Modellbeschreibung soll durch den Vergleich von synthetischen mit realen Beobachtungen untermauert werden.

Methoden: Wir treiben das 3D MHD Model mit beobachteten photosphärischen Magnetfeldern und Horizontalbewegungen an. Durch Wärmeleitung entlang des Feldes sowie Strahlungsverluste wird die koronale Energiebilanz realistisch. Wir synthetisieren Spektren in verschiedenen Emissionslinien mit einer Atom-Datenbank und der berechneten koronalen Plasmatemperatur sowie -dichte. Diese vergleichen wir mit entsprechenden Beobachtungen der Korona über der aktiven Region, mit der wir die Simulation antreiben. Wir vergleichen extrahierte Modell-Feldlinien mit empirischen und theoretischen Skalengesetzen, die die koronale Heizung entlang von Bögen voraussagen.

Resultate: Im Modell bilden sich heiße koronale Bögen mit Temperaturen deutlich über $1 \mathrm{MK}$. Ihre 3D-Struktur entspricht den beobachteten koronalen Bögen; Doppler-Karten lassen auf ähnliche Plasmaströmungen entlang der Bögen schließen. An die Modell-Daten passen wir ein Skalengesetz an, welches von der Bogenlänge und der magnetischen Flussdichte an den Fußpunkten abhängt.

Schlussfolgerungen: Aus der substanziellen Übereinstimmung zwischen Modell und Beobachtung schließen wir, dass das Modell eine genügende Beschreibung der Heizung und Wärmeleitung entlang von koronalen Bögen darstellt, um die Beobachtungen zu erklären. 



\section{Summary}

Context: The corona of the Sun can be observed since 1932 with instruments occulting the solar disc. Only few years later it became clear that the corona is way hotter than the visible solar surface and since then, the coronal heating mechanism is unclear. So far, many processes have been proposed that are able to deliver enough energy to the base of the corona, but no complete and consistent picture of the energy transport and its localized dissipation in the corona is established.

Aims: We aim for a self-consistent model of driving motions at the solar surface that bend and braid the magnetic field in the corona and produce heat by Ohmic dissipation of induced currents. We want to justify our model description by deducing synthetic observations that we check against real observations.

Methods: We use observations of the magnetic field in the photosphere, as well as horizontal photospheric motions to drive our 3D MHD model. Field-aligned heat conduction and radiative losses allow for a realistic coronal energy balance. We deduce synthetic spectra in different emission lines with an atomic database using the computed coronal plasma temperature and density. These we compare with the corresponding observations of the corona above the same active region that we used for the driving. We compare samples of field lines extracted from the model corona with empirical and theoretical scaling laws predicting the coronal heating along loops.

Results: Hot coronal loops of temperatures well above $1 \mathrm{MK}$ form in the model corona. Their 3D structure matches the observed coronal loops and coronal Doppler shift maps indicate similar plasma flows within the observed and the model loops. With a fit to the model data, we find a scaling law that relates to the loop length and its foot-point magnetic flux density.

Conclusions: From the substantial match between our model and the observed corona, we conclude that the model provides a sufficient description of the heat input and conduction along coronal loops to explain diverse observations. 



\section{Introduction}

\section{Sunna, Aton, Inti, Mitra, Huitzilopochtli, Amaterasu, Wi, Helios, Svarožić, Sol, Utu}

The Sun is an average star of the spectral class G (Unsöld and Baschek 2002) far out in a totally unfashionable spiral arm of our host galaxy (Adams 1979). What turns this yellow unregarded object so "sexy" for Astronomers and Astrophysicists is not so much the fact that the Sun is the only star we can observe in high spectral and spatial resolution, but that it features magnetic fields. Since millennia humans are able to observe sunspots, that are dark features in the visible light range. The magnetic fields were first observed inside sunspots and were correctly interpreted as such by Hale (1908a) with magnetic flux densities up to $2900 \mathrm{G}$ (Hale 1908b). At the visible surface of the Sun (the photosphere) the density drops very quickly and the atmosphere of the Sun becomes increasingly transparent, see Fig. 1.1. Above the photosphere there is a temperature minimum of $4000 \mathrm{~K}$. Towards the outer atmosphere the temperature rises again, instead of just becoming cooler, as one would expect in the first place when going away from a heat source. This temperature rise begins in the chromosphere, reaching to about $20^{\prime} 000 \mathrm{~K}$, and then takes a steep rise in the transition region (TR), before reaching to 1'000'000 K and more in the corona.

The corona gives riddles since about 80 years: Lyot (1932) built the first imaging instrument to observe the corona without the requirement for a natural total solar eclipse and found a polarization signal in the corona; Grotrian (1934) reported inexplicable high electron velocities, while Alfvén (1941) first concludes correctly the high coronal temperature, and Edlén (1943) first reported about strong ionization in the corona. Without the magnetic field, the Sun would only show the thermal convection ending in the photosphere (called granulation) and an atmosphere in hydrostatic equilibrium. But, because the corona is heated to high temperatures, it drives a continuous flow equilibrium (Parker 1963), also known as the solar wind. This particle and plasma flow from the Sun counteracts the galactic wind, coming from other stars in our host galaxy, inside a region that is called the heliosphere 


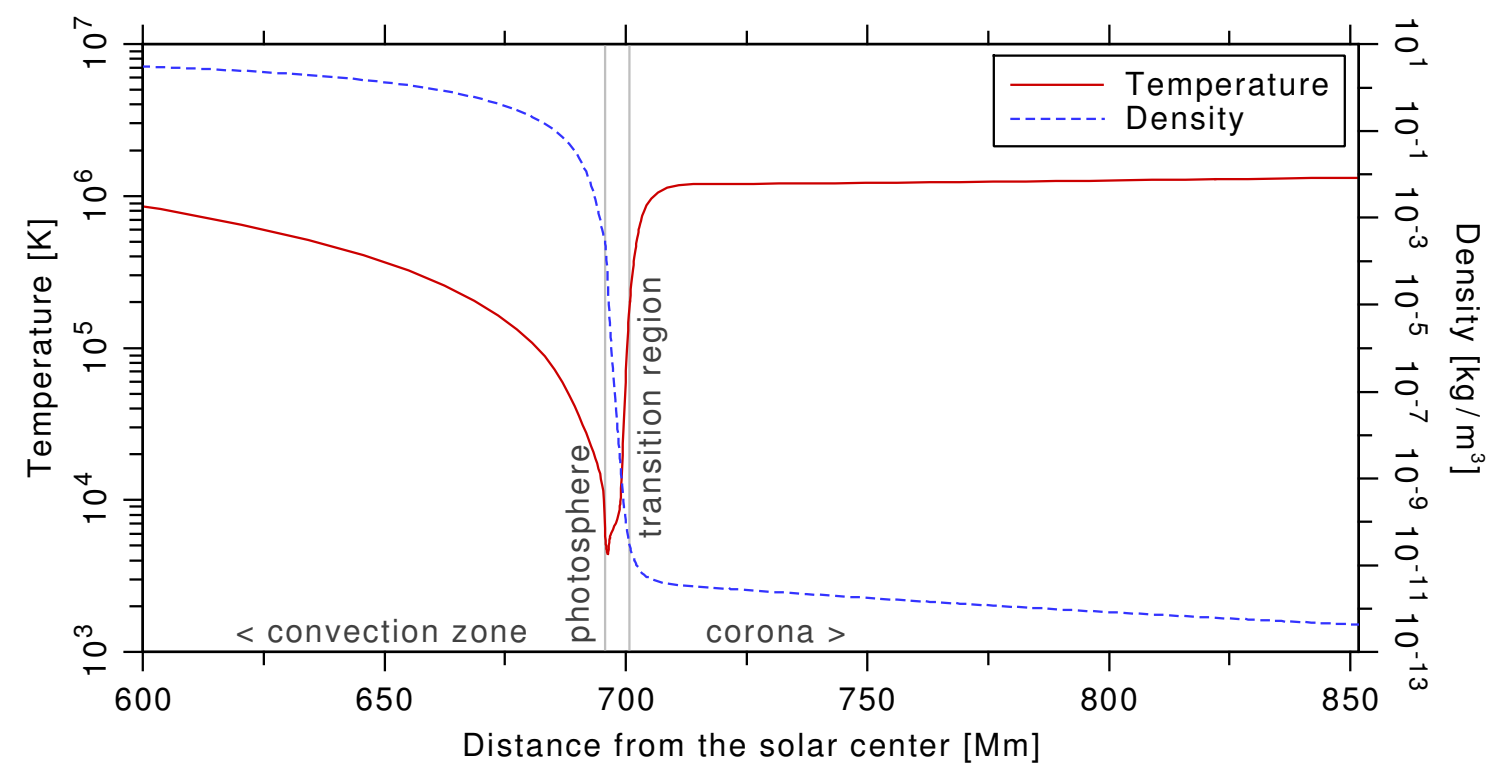

Figure 1.1: Temperature (red solid line) and density stratification (blue dashed line) of the solar atmosphere. This stratification is a combination of a $1 \mathrm{D}$ model of the solar interior (Stix 2004), a ID radiative transfer model from the photosphere to the upper chromosphere (Fontenla etal. 1990), and a smooth transition from the chromosphere to coronal densities and temperatures inferred from observations (November and Koutchmy 1996). The gray vertical lines indicate the position of the photosphere and the transition region.

and that extends even over the orbits of the outer planets. Therefore, our host planet Earth and its magnetic field is influenced by the solar wind (Axford 1962). Our society, that is increasingly relying on large-scale electric networks, satellite technology, and that currently has a manned station in space, needs to care about the space weather (Schwenn 2006).

Due to the processes creating magnetic flux concentrations in the interior of the Sun, which are sill under debate (Charbonneau 2005), we observe not only sunspots with strong magnetic fields, but also a magnetic network with spatial scales larger than the granulation (Babcock and Babcock 1955), as well as a magnetic "salt-and-pepper" pattern of strong opposite polarity field (Beckers and Schröter 1968) all over the solar surface. The smallest magnetic structures are still spatially unresolved even with the most recent solar telescopes. The quiet Sun (QS) has an average magnetic flux density of about $100 \mathrm{G}$ and due to its magnetically "open" structure (mainly radial field in the corona) particularly the fast solar wind is formed above the QS.

Besides the isolated sunspots (and the much smaller magnetic pores) also 


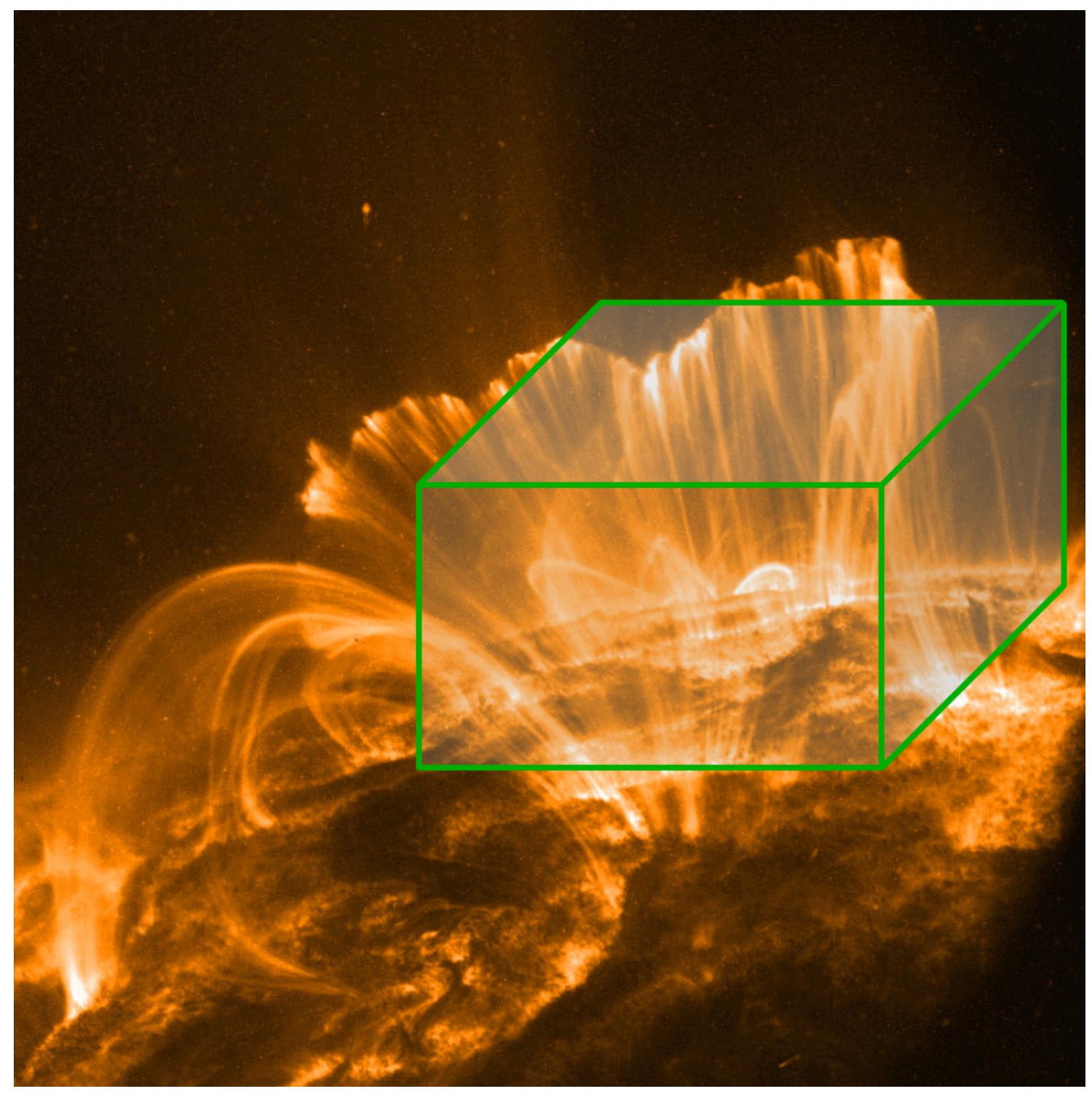

Figure 1.2: The solar coronal emission above a magnetically active region in the spectral range of FeIX and FeX; image taken by the TRACE space observatory (Strong etal. 1994). The small bright roughly semi-circular structures are hot coronal loops that produce EUV emission and that span between regions of opposite magnetic polarity in the photosphere. The larger coronal loops are less bright structures spanning high up into the corona that usually are cooler than the hot $A R$ core. The spatial extend of the computational domain, that is supposed to cover both types of coronal loops, is overplotted as green cuboid. Image credit: NASA/LMSAL.

magnetically active regions (AR) appear in the photosphere. ARs are concentrations of magnetic flux with similar field strengths as sunspots, which therefore often host groups of sunspots with opposite magnetic polarity. The corona above ARs is highly 
dynamic and is usually dominated by closed field that retains the plasma on place longer than above the QS, so that it can be heated more intensively as above the QS. Accordingly, the solar wind emerging in ARs is slower. Hot AR loops that emit EUV light and X-rays form along the coronal magnetic field, see Fig. 1.2 . From this introduction, it already becomes clear that the high temperature in the corona is fundamentally important for the dynamical processes in the heliosphere. Because of the temperature stratification that only allows thermal energy to flow away from the corona, the corona needs to have an energy source. As the corona is optically thin for light, high-energy photons, and neutrinos, there are few alternatives to play an important role in the heating of the corona. One of them are the solar magnetic fields, including electromagnetic waves.

Understanding the structure and dynamics of the corona is a prerequisite to understand the dynamic outbreaks of the sun, like coronal mass ejections and prominences, and hence to understand and predict the continuously changing conditions in the heliosphere.

\section{The coronal energy source}

So far, the coronal heating mechanism is barely understood, although various theories exist - many of them are listed in the review of Klimchuk (2006). Often, the coronal energy input is only described up to the base of the corona (Mclntosh et al. 2011: Wedemeyer-Böhm etal. 2012), not including the feasibility of the necessary dissipation process in the corona.

Even though the corona above ARs is known to loose about $10^{\prime} 000 \mathrm{~W} / \mathrm{m}^{2}$ via heat conduction and radiative losses (Withbroe and Noyes 1977), we more face a problem in describing a consistent theory of an energy transport and its actual dissipation mechanism in the corona, than just possible mechanisms for a sufficient energy supply to the base of the corona. Dissipation mechanisms are mainly grouped in a) wave heating (AC), which leaves the problem that most waves are either reflected back at the base of the corona or pass the corona without much effect (Narain and Ulmschneider 1996), and b) a steady propagation of magnetic stress energy (DC). Other ways would be $c$ ) the pass-through of shock waves after large and short-lived reconnection events, but shock-wave generating flare events are relatively rare phenomena (Švestka 1956), and d) hydrodynamic shock waves from the solar surface are quickly dissipated already in the chromosphere before reaching the corona (Athay and White 1978).

Basically, $A C$ heating occurs if the state-change in the magnetic field is faster than the relaxation in the topology of the magnetic field, and DC heating otherwise. The critical limit is set by the Alfvén velocity, e.g. any advecting motion faster than this 


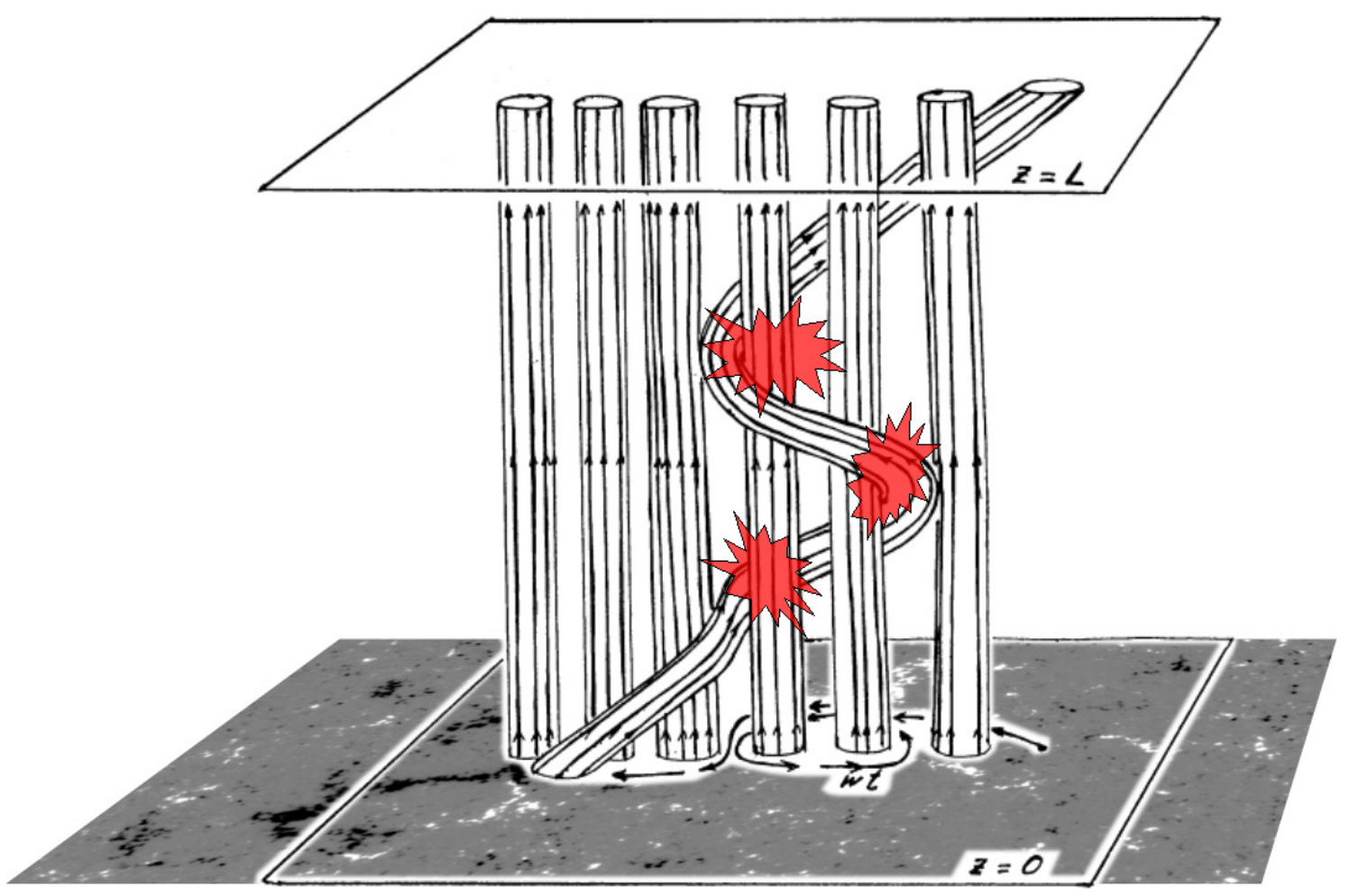

Figure 1.3: Sketch of the photospheric magnetic field that is braided by stochastic horizontal motions due to the granulation in the photosphere (Fig. 1 in Parker, E. N. 1983). The AR field lines are rooted in the photosphere $(z=0)$ and connect to another location of opposite polarity in the photosphere $(z=L)$. Electric currents occur in regions of strongly bended field lines or due to diffusional cancellation of opposite oriented magnetic flux.

velocity will result in transversal or longitudinal waves propagating along the field. Current research on $\mathrm{AC}$ heating concentrates on waves ignited in the corona due to fast magnetic reconnection, propagated and dissipated again in the corona (Moore et al. 1991; Sturrock 1999). Even though observational evidence exists for upwards propagating acoustic waves in radial field lines (Berghmans and Clette 1999) and for intensity fluctuations in the lower parts of coronal loops (De Moortel et al. 2002), many ARs are relatively stable, at least during most of their lifetime, so their heating mechanism should be persistent. Another idea still under discussion are impulsive heating events from small reconnection events called nanoflares (Parker 1988), which is a candidate to explain non-thermal spectral line broadenings by unresolved motions due to plasma expansion following on intermittent heating (Patsourakos and Klimchuk 2005).

A coronal heating mechanism including the actual dissipation is the field-line braiding due to horizontal shuffling motions in the photosphere that leads to 
induced currents in the corona, where these currents heat the plasma through Ohmic dissipation (Parker 1972). In Fig. 1.3 we sketch this mechanism, where the upper and lower layer both reflect the photosphere, meaning that the driving motions can entangle the coronal magnetic field from both ends of the field lines. We speak of "nanoflares" when the electric currents dissipated in the corona follow on short-lived reconnection events. In the case of quasi-stationary magnetic field reconfiguration we speak of "magnetic diffusion". Downscaled models of solar active regions indicated that this mechanism can produce a loop-dominated corona (Gudiksen and Nordlund 2002 2005ab; Bingert et al. 2010).

We have conducted a 3D magnetohydrodynamics (MHD) numerical simulation of the solar corona above a magnetically active region (AR). The employed model includes gravity, radiative losses following Cook et al. (1989), field-aligned heat conduction (Spitzer 1962), and a uniform magnetic diffusivity $\eta=10^{10} \mathrm{~m}^{2} / \mathrm{s}$. The plasma pressure and the magnetic field in the corona are described self-consistently. To drive our model from the bottom we use photospheric observations, i.e.

Hinode/SOT (Kosugi et al. 2007; Tsuneta et al. 2008) line-of-sight magnetograms and horizontal velocities. Large-scale motions of magnetic patches and

granulation-like small-scale velocities introduce magnetic stress at the magnetic field footpoints. These photospheric perturbations propagate along the magnetic field into the corona and carry energy (e.g., Poynting flux).

One approach to understand and describe the heating of the corona is to statistically derive scaling laws from observed coronal loops that relate global loop properties like their maximum temperature, their length, their density, or the magnetic field at their footpoints with the heating rate (Rosner et al. 1978; Serio et al. 1981; van Ballegooijen et al. 2011), irrespective of how exactly this heating is produced. Such scaling laws have also been used to drive coronal models (e.g., Lionello et al. 2005) and to test the observable consequences of different scaling laws on the corona (van Wettum et al. 2013). With observations of an AR and a numerical experiment that we have proven to match observations (Bourdin et al. 2013), we are now able to test theoretical scaling laws (that relate global coronal loop parameters with their heating) for consistency with our model corona. Thus, we have the possibility to follow the magnetic field through the model corona and extract any quantity along that field line, which is otherwise inaccessible by coronal observations.

With the atomic database CHIANTI (Dere et al. 1997; Young et al. 2003) we deduce the synthetic emission following Peter et al. (2004 2006), see Fig. 1.4. We compare the synthetic spectra to real observations of the same AR, like the EUV and X-ray emission spectra from highly ionized atoms and their corresponding Doppler line-shifts, taken by Hinode/EIS (Culhane et al. 2007). 


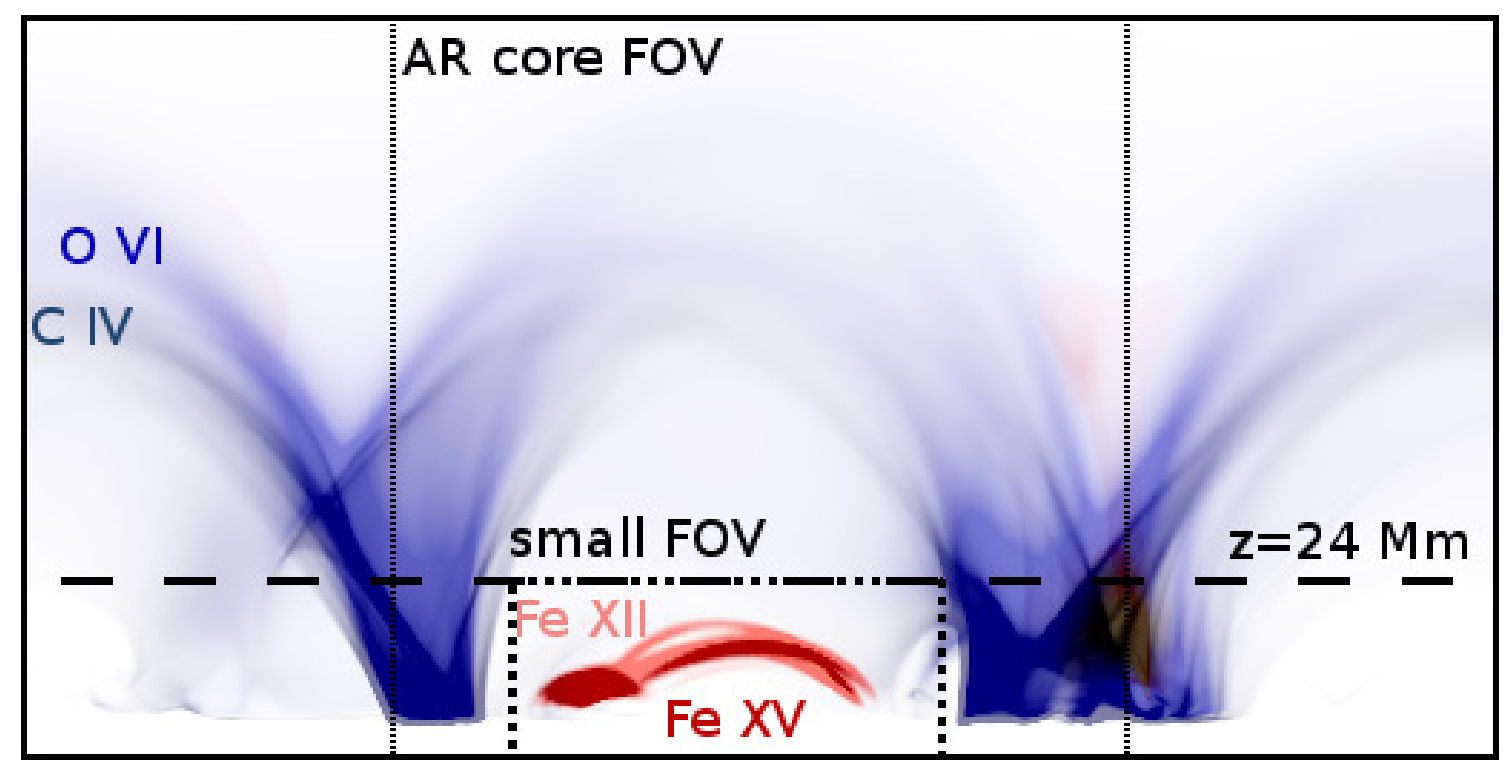

Figure 1.4: Synthetic composite image of four emission lines (in linear scale) formed blow 0.2 MK (C IV, O VI) and above $1 \mathrm{MK}$ (FeXII, FeXV), integrated along a horizontal line-of-sight, as if the model AR would have been observed on the limb of the solar disc. In the AR core a hot coronal loop system if found that shows emission of plasma at $1 \mathrm{MK}$ and more. Larger and cooler loops are found to span over the hot $A R$ core, as found in observations, c.f. Fig. 1.2 . This image was generated from a snapshot at minute 63 in our simulation run, c.f. Fig. 2.3.

Statistical analyses of synthesized spectra from these models matched the statistical properties of observed spectra (Peter et al. 2004 2006). The Doppler shifts in such models were able to reproduce the observed persistent redshifts in the TR (Peter 1999). So far, either AR models showed net redshifts in the hot coronal emission lines above the TR (Zacharias etal. 2011ab), or models of QS could reproduce the TR redshift together with a turnover versus zero Doppler shifts in the hotter coronal lines (Hansteen et al. 2010). Still, no model could reproduce significant blueshifts in hot coronal lines, e.g. Fe XV, that the observations show. We are presenting here a model of an $A R$ in a full-scale computational domain that implements the field-line braiding process and is able to reproduce both, the redshifts in the TR and the blueshifts in the corona (Chap. 5). 


\section{Outline}

First, we introduce our model setup in Chap. 2. We found a substantial match of coronal structures and their flow dynamics between the simulated and observed loops system, see Chap. 3. Based on these results, we test scaling laws of the coronal heating for their consistency with our model and fit a new scaling law to our data in Chap. 4. Finally, in Chap. 5] we address the "contradiction" of persistent TR redshifts and coronal blueshifts. 


\section{Technical introduction}

\subsection{Treatment of space plasmas}

For a general introduction into plasma and space-plasma physics, we refer to one of the many good text books, like Boyd and Sanderson (2003); Kippenhahn and Möllenhoff (1975); Priest (1982); Aschwanden (2004). The properties of a plasma, or a magnetized electrically conducting fluid, can, under certain assumptions, be described with the set of magnetohydrodynamical (MHD) equations.

\subsubsection{Magnetohydrodynamics}

The MHD equations are derived from the Maxwell equations, including the Lorentz force, the hydrodynamical continuity equation, the conservation of mass and impulse, and Ohm's relation. The thermal energy balance we obtain by multiplying Ohm's relation with the current density $\vec{j}$, adding the terms for the dissipated energy by the kinematic and the bulk viscosity, as well as subtracting the radiative losses and heat conduction terms. To relate the plasma pressure $P$ with the density $\rho$ and the temperature $T$, we use the ideal gas law.

Assumptions in MHD are: 1) the negligence of the displacement currents, which reduces the $\vec{\nabla} \times \vec{B}$ term in the Maxwell equations to only Amperes law, 2) the quasi-steady state changes of any of the described quantities, and 3 ) the high conductivity and hence the quasi-neutrality of the plasma. This last point eliminates any terms containing the charge density from the Maxwell equations.

\subsubsection{Quasi-neutrality}

We want to inspect the assumption of quasi-neutrality in a volume of interest, i.e. the grid cell volume. Due to thermal fluctuations in the electron density, a localized surplus of positive or negative charges is eventually created in a plasma. To estimate the applicability of the quasi-neutrality assumption, we can estimate a volume size that would be at least close to neutrality because of the large number of particles contained in that volume. Once a volume is charged, it has an electric 
field that is proportional to the electric charge contained in that volume. This electric field will attract the charges that are missing in that volume and hence the neutrality will partly be regained, as long as that would not disturb the neutrality of the surrounding plasma or other forces hinder the thermal diffusion of electrons. The length scale of persistently charged volumes is given by the Debye length of electrons $\lambda_{e}$ that is set by the electron temperature $T_{e}$, as well as the electron particle density $n_{e}$ :

$$
\lambda_{e}=\sqrt{\frac{\epsilon_{0} k_{B} T_{e}}{\pi e^{2} n_{e}}}
$$

$k_{B}$ is the Boltzmann constant, $\epsilon_{0}$ the vacuum permittivity, and $e$ is the electron charge. Quasi-neutrality can only be maintained on length scales larger than the Debye length. For the photosphere, the Debye length is about $\lambda_{e, p h o t}=2 \mu \mathrm{m}$ and in the corona it can reach up to $\lambda_{e, c o r}=1 \mathrm{~m}$. Besides that, the electron gyro radius in the corona is about $20 \mathrm{~m}$. Our model grid spacing is much larger than these microphysical process scales, so that we can safely use the MHD equations.

\subsubsection{Plasma beta}

To estimate the dominance of either the magnetic field or plasma motions over the other, we have to consider the inertial force, the Lorentz force, and the viscosity. As we already assumed only quasi-steady state changes, inertia is of low relevance. We can concentrate on the ratio between the electromagnetic and the kinematic pressure (or equally the respective energy densities). This defines a dimensionless number called plasma beta:

$$
\beta=\frac{2 n k_{B} T}{B^{2} / \mu_{0}}=\frac{\rho u_{k i n}^{2}}{B^{2} / \mu_{0}}
$$

In the photosphere, we compute the plasma beta with the photospheric density of about $\rho_{\text {phot }}=3 \cdot 10^{-4} \mathrm{~kg} / \mathrm{m}^{3}$ (c.f. Fig. 1.1 ) and typical velocities due to the granulation of about $\vec{u}_{\text {phot }}=1000 \mathrm{~m} / \mathrm{s}$ to $\beta_{\text {phot }}=4$ for an average magnetic flux density of $\vec{B}_{\text {phot }}=100 \mathrm{G}$. Inside sunspots with flux densities of $\vec{B}_{\text {spot }}=2000 \mathrm{G}$ we obtain $\beta_{\text {spot }}=0.01$. This means that magnetic flux concentrations are advected with the photospheric motions, except for sunspots where the strong field suppresses the thermal convection. Therefore, a sunspot is cooler and darker than its surrounding. In the corona, with a density of about $\rho_{c o r}=10^{-13} \mathrm{~kg} / \mathrm{m}^{3}$ and large velocities of about $\vec{u}_{c o r}=250 \mathrm{~km} / \mathrm{s}$, we get even for a small flux density of only $\vec{B}_{\text {cor }}=2 \mathrm{G}$ a plasma beta of $\beta_{\text {cor }}=0.2$. Hence, the plasma motions in the corona are dominated by the magnetic field topology. 


\subsubsection{The equations}

In our numerical computation we use the following form of the MHD equations, consisting of the continuity equation (2.1.3), the equation of motion (2.1.4), the induction equation (2.1.5), and the energy balance (2.1.6):

$$
\begin{aligned}
\frac{\mathrm{D} \ln \rho}{\mathrm{D} t}= & -\vec{\nabla} \cdot \vec{u} \\
\frac{\mathrm{D} \vec{u}}{\mathrm{D} t}= & -c_{S}{ }^{2} \vec{\nabla}\left(\frac{s}{c_{P}}+\ln \rho\right)-\vec{\nabla} \Phi_{\text {grav }}+\frac{1}{\rho} \vec{j} \times \vec{B}+ \\
& +v\left(\nabla^{2} \vec{u}+\frac{1}{3} \nabla \nabla \vec{u}+2 \mathcal{S} \cdot \vec{\nabla} \ln \rho\right)+\zeta(\nabla \nabla \vec{u}) \\
\frac{\partial \vec{A}}{\partial t}= & \vec{u} \times \vec{B}-\mu_{0} \eta \vec{j} \\
\rho T \frac{\mathrm{D} s}{\mathrm{D} t}= & \mu_{0} \eta j^{2}+\vec{\nabla} \cdot\left(\kappa_{\text {Spitzer }} \vec{e}_{B} \cdot\left(\vec{\nabla} T \cdot \vec{e}_{B}{ }^{T}\right)\right)- \\
& -L_{\text {rad }}+2 \rho v \mathcal{S} \odot \mathcal{S}+\zeta \rho(\nabla \cdot \vec{u})^{2}
\end{aligned}
$$

with the unit vector along the filed $\vec{e}_{B}$, the sound speed $c_{S}$, the kinematic viscosity $v$, the bulk viscosity $\zeta$, the magnetic diffusivity $\eta$, the vacuum permeability $\mu_{0}$, the heat conduction coefficient $\kappa$, the specific heat at constant pressure $c_{P}$, the gravitational potential $\Phi_{\text {grav }}$, and the rate-of-strain tensor $\mathcal{S}$ that is here traceless. It is of crucial importance to get the energy balance as realistic as possible, including all relevant energy sources and sinks. The Ohmic heating term is contained in Eqn. 2.1.6 as:

$$
H_{O h m}=\mu_{0} \eta j^{2}
$$

The radiative loss function $L_{\text {rad }}$ we take from Cook et al. (1989). The field-aligned Spitzer-type heat conduction (Spitzer 1962) is implemented as

$$
\kappa_{\text {Spitzer }}=1.8 \cdot 10^{-10} \frac{(T /[\mathrm{K}])^{5 / 2}}{\ln \Lambda}[\mathrm{W} /(\mathrm{m} \mathrm{K})]
$$

using a Coulomb logarithm of $\ln \Lambda=20$ that represents typical coronal densities and temperatures.

We use in all equations the magnetic vector potential $\vec{A}$ and derive the magnetic field as $\vec{B}=\vec{\nabla} \times \vec{A}$. With that, we automatically keep the magnetic field divergence free.

The computation is performed using also the logarithmic density $\ln \rho$ and the logarithmic temperature $\ln T$ that is proportional to the entropy $s$. This choice is 


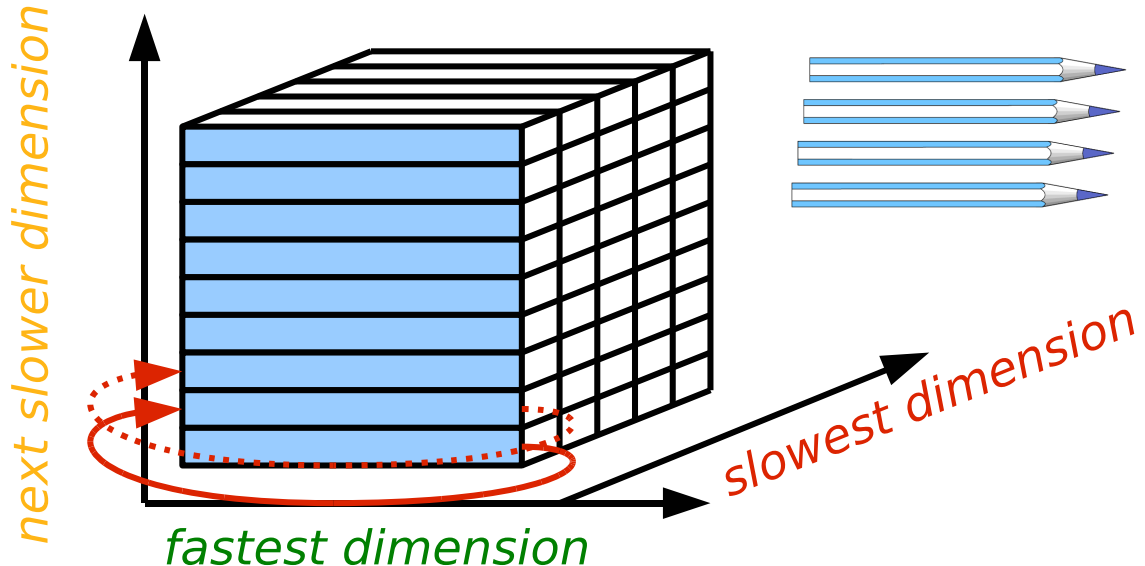

Figure 2.1: Data alignment concept of the Pencil Code within one processor's subvolume of the 3D model, see Sect. 2.2.

necessary to cope with the steep gradients in both these quantities in the TR, and especially with the large drop of about 8 orders or magnitude in the density that we have within our model (c.f. Fig. 1.1).

\subsection{The code}

We use the Pencil Code $\rrbracket^{\top}$ that implements the MHD equations with a $6^{\text {th }}$-order finite-differences numerical scheme. For the temporal integration we use a classical $4^{\text {th }}$-order Runge-Kutta method with a variable time step. The Pencil Code got its name from its memory alignment scheme, where all equations are computed in "pencils" containing the data in elongated arrays along the fastest dimension of the programming language FORTRAN, which makes the code robust and highly efficient regarding the usage of processor cache and the vector computation unit, see Fig. 2.1 .

We use MPI to run the model setup typically on 1024 processor computing cores in parallel, see the scaling plot in Fig. 2.2. Our simulation domain is divided in elongated cuboid subdomains to maintain most of the cache and vectorization efficiency and in the same time find a good volume-surface ratio for the subdomains, so that the boundary layer communication between the subdomains is optimized. The communication of the subvolume boundaries is arranged so that the code can continue with the computation of independent "inner" pencils, while the boundary data is transferred asynchronously.

\footnotetext{
' http://Pencil-Code.Nordita.org/
} 


\section{JuRoPA Benchmark}

Scalability of the Pencil Code regarding number of cores for the proposed setup

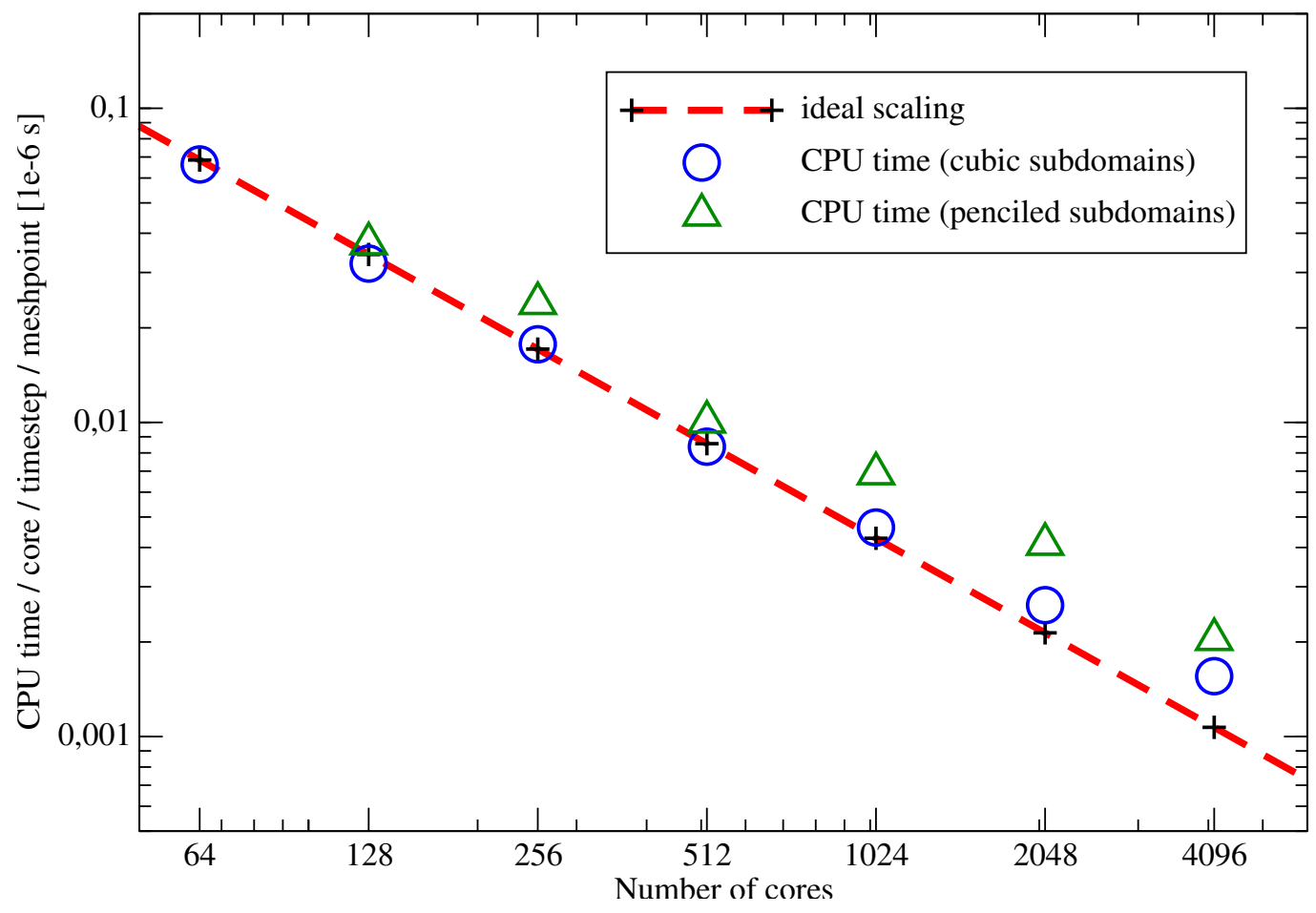

Figure 2.2: Scalability plot of a fixed-size 3D model setup ("hard scaling") on the supercomputer JuRoPA, see Sect. 2.2. The ideal scaling (red dashed line) indicates an exponent of 2 . Within this work the scalability was extended by a factor 8 .

For the file input and output we use 16 IO-nodes that collect the data in xy-layers and write the combined data in parallel. Configuration files and logfiles are read and written only from one root IO-node. This allows networking filesystems (such as LUSTRE or GPFS) to cope with the high demand of simultaneous file access.

Our magnetic field boundary condition needs to compute a Fourier transform in both horizontal directions. For that, we remap the data in the uppermost and lowermost layer of the domain from the subvolume into pencil shape. After the transform, the result is transported back to the respective subvolume. This way of computation turns out to be faster than changing the subvolume setup to the elongated pencil shape for the whole 3D setup (c.f. Fig. 2.2). 


\subsection{Coronal model setup}

\subsubsection{Basic idea}

The basic idea of this work is to set up a forward model of the solar corona above an $A R$ based on photospheric observations. We decide for a setup in full spatial scale and with as high spatial resolution as observations are available, which requires us to use high-performance computing sites. In principle, we self-consistently compute all plasma properties on a 3D grid, like the pressure, the magnetic field, and the flow dynamics in the corona. Most of those quantities are usually inaccessible by coronal observations.

Two essential components are needed to get a realistic coronal energy balance: a) the Spitzer-type heat conduction parallel to the magnetic field (Spitzer 1962) and b) the radiative losses of the optically thin corona (Cook et al. 1989). These are the two main energy sinks in the corona that need to be compensated by a realistic coronal heating mechanism.

For the lower part of the atmosphere, up to the upper chromosphere, we use a prescribed heating and cooling function to mimic the effects of chromospheric heating and radiative transfer processes on the atmospheric stratification. These processes are not self-consistently contained in this model, because we need the lower part of the atmosphere only as a "flexible" boundary condition - in other words as a mass and thermal energy reservoir for the corona (c.f. Fig. 1.1).

\subsubsection{Model parameters}

The simulation domain covers the spatial scale of the observed $A R$ together with some surrounding QS, which is $235 \cdot 235 \cdot 156 \mathrm{Mm}$ covered by

$1024 \cdot 1024 \cdot 256$ grid points. In the vertical direction the non-equidistant grid distance varies from $100 \mathrm{~km}$ below the TR to $800 \mathrm{~km}$ in the upper corona up to $156 \mathrm{Mm}$ height. This large extent in the vertical direction is necessary, because we need to encompass roughly semi-circular loops with footpoint distances up to $200 \mathrm{Mm}$ and in the same time we need to stay independent of the upper boundary.

We use a density diffusion of $D_{\rho}=20 \cdot 10^{6} \mathrm{~m}^{2} / \mathrm{s}$ and an isotropic heat conduction of $\chi=500 \cdot 10^{6} \mathrm{~m}^{2} / \mathrm{s}$ that is way less efficient than the Spitzer-type heat conduction, but provides numerical stability also across the magnetic field direction. The magnetic diffusion constant we use is uniform and constant in the coronal domain and we set it to $\eta=10^{10} \mathrm{~m}^{2} / \mathrm{s}$, which is needed for numerical stability and is much larger than the physical value of about $100 \mathrm{~m}^{2} / \mathrm{s}$ in the solar corona. But, 
the smallest dissipation scales in the corona correspond to some $20 \mathrm{~m}$, which is the electron gyro radius in coronal plasma. As we use a grid spacing of $230 \mathrm{~km} / \mathrm{s}$ horizontally, the simplest approach to model the sub-grid dissipation is by a diffusion equation with a diffusion constant that corresponds to the real diffusion constant and dissipation length scales. Because $\eta$ is proportional to the length scale squared, our numerical diffusion constant and grid spacing fit to the physical ones. By that, we assume that any turbulent cascade to the microphysical dissipation scales actually is representable by a diffusion equation. This is a weak point of our model, but if the model reproduces the observations well, this assumption is justified.

In other words, our choice of $\eta$ sets the diffusion scale and the viscosity $v=10^{10} \mathrm{~m}^{2} / \mathrm{s}$ sets the velocities that our model can cope with. In this case the viscosity is similar to estimates of the physical coronal viscosity (Priest 1982). The numerical scheme we use supports grid Reynolds numbers (based on the grid spacing as typical length scale) up to 5 , which means that the model is numerically still stable for magnetic structures as thin as $1 \mathrm{Mm}$ width and for velocities of at maximum $500 \mathrm{~km} / \mathrm{s}$. During the simulation run these stability criteria were fulfilled. Further information on the model setup is provided in Sect. 3.3.1 and the full simulation parameter set is publicly available (Bourdin et al. 2013).

\subsubsection{Initial condition}

\subsubsection{Stratified atmosphere}

The initial condition of the atmosphere consists of a stratified atmosphere close to hydrostatic equilibrium. The numerical density diffusion parameter we use to stabilize the computation numerically, provides for a slight "virtual" mass transport along the gradient in density. This acts against the hydrostatic equilibrium and needs to be compensated by a slight counter flow in opposite direction, resulting in a flow equilibrium between upwards diffusion and downwards plasma bulk motion. When we want to relax an initial temperature stratification, we need to compute first the density that fits to an analytical hydrostatic initial condition. Because the analytic derivatives and integrals are exact and the numerical ones are not, an analytical solution for the barometric equation does not match the numerical equilibrium state. Therefore, such initial conditions ignite small amplitude compressional waves that propagate into the computational domain. The solar atmospheric density drops by many order of magnitude, so that such compressional waves reach huge amplitudes, when they eventually reach the low-density regime of our model. This we circumvent by finding the numerical equilibrium state in a ID hydrodynamic simulation, using the same diffusion parameters as in our 3D 
model, starting from partly observed and theoretically derived density and temperature stratifications of the Sun and its atmosphere (c.f. Fig. 1.1).

After many hours solar time, which is much longer than our 3D model is supposed to run, the initial perturbations have relaxed. We construct our initial stratified atmosphere from the obtained ID numerical flow equilibrium. Even though the remaining velocities are of the order of $\mu \mathrm{m} / \mathrm{s}$, we also have to transfer these velocities to the $3 \mathrm{D}$ model initial condition, because otherwise we would again disturb the numerical flow equilibrium and ignite compressional waves again, because a net force would be present in the initial condition. A velocity damping alone would not help to suppress these compressional waves, because they are anyway slow and of low amplitude, but get amplified tremendously once they cross the steep gradient in density and reach the low densities in the corona.

\subsubsection{Initial magnetic field}

The initial condition for the magnetic field consists of a potential-field extrapolation from the observed photospheric magnetogram (see right panel in Fig. 3.1). Any perturbations from the driving at the lower boundary need at least the Alfvén crossing time to reach the corona and to heat in-situ by Ohmic dissipation of currents. For that reason we smoothly switch on the radiative losses and the heat conduction at later stages in the simulation. Not doing so would result in a collapsing corona, because the cooling would be immediate while the heating is delayed.

\subsubsection{Switching on}

In Fig. 2.3 we show the time series of the maximum temperature, the average Ohmic heating per particle, and the average viscous heating per particle inside the subvolume that contains the hot AR core loops. The Spitzer-type heat conduction smoothly sets in starting with minute 10 and is fully active at minute 30 , this allows for a larger time step at the beginning of the simulation. The radiative losses are (in the same way) smoothly switched on from minute 15 to 35 , that is to compensate for the missing part of the Ohmic heating in the coronal energy balance before any magnetic perturbations from the lower boundary have reached the corona. A strong velocity damping is used to relax any disturbances in the atmospheric stratification that might have arisen from any switch-on effects, like the insertion of the magnetic field and changing model parameters. The velocity damping is smoothly switched off with a cubic step function over a time interval spanning from minute 20 to 40 . 


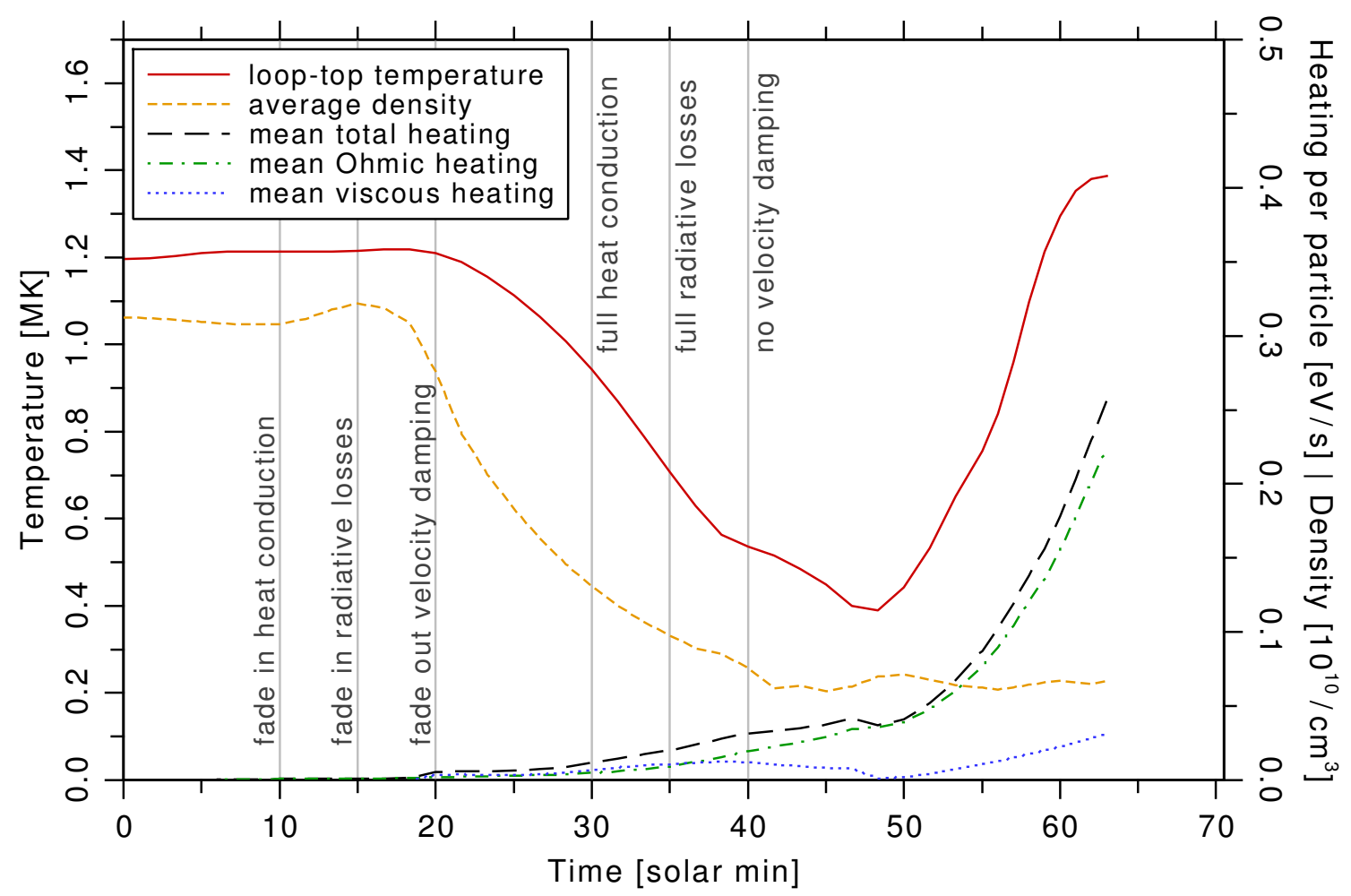

Figure 2.3: Time-series of the maximum temperature and heating terms in the $A R$ core subvolume containing the hottest loop in the model (c.f. CL 1 and SL 1 in Bourdin et al. 2013). We show here the maximum temperature within the subvolume (red solid line) together with the average Ohmic heating rate per particle (green dashdotted) and the average viscous heating rate per particle (blue dotted). The total of both heating terms we show as black dashed line. The orange short-dashed line indicates the average density of the coronal part of the hottest loop (SL 1). Please refer to Sect. 2.3.3.3 for a description of the vertical gray lines.

\subsubsection{Self-consistent model}

After 40 minutes, all physical terms are fully active and the velocity damping has faded out. Now the system evolves self-consistently. At minute 48 strong Ohmic heating sets in and the maximum temperature in the subvolume increases rapidly (c.f. Fig. 2.3). This time roughly corresponds to three life-times of a granule ( 5 minutes each) plus the Alfvén crossing time into the corona (about 30-40 minutes). We see how the radiative losses cooled down the whole computational domain by dissipating some of the initial atmospheric internal energy. This might first look like an unwanted and avoidable effect, but it allows us to conclude that any later temperature increase is not caused by the initial condition, but explicitly caused by a high in-situ Ohmic heating per particle. 
Even though the Ohmic heating is still rising, the maximum temperature reaches a kind of plateau, see Fig. 2.3. A further rise in the Ohmic heating would not result in significantly higher temperatures, because the radiative losses and the Spitzer-type heat conduction would both become much more efficient, as they both depend on the temperature in a non-linear way and would therefore stabilize the plateau temperature. The hot smaller loops SL 1-3 have formed about 8 solar minutes before the larger loop CL 1 and are hence in a stable and sufficiently evolved state.

\subsubsection{Boundary conditions}

Our model is periodic in the horizontal directions. The lower and upper boundary of the computational domain are closed for any plasma inflow or outflow, and the temperature is chosen to be symmetric at the boundaries to avoid any artificial energy flow into or out of the domain. The density is set according to the temperature, so that the boundary of the physical domain is in hydrostatic equilibrium. We extrapolate the magnetic field at the upper boundary with a potential field into the three ghost layers.

\subsubsection{Photospheric magnetic field}

For the magnetic field at the lower boundary, we use a potential-field extrapolation into the interior of the Sun, which is in principle identical to a potential-field extrapolation to the exterior, just that we don't decrease the contrast exponentially depending on the distance from the physical boundary layer, but we increase the contrast of the vertical magnetic field with depth. The pressure scale height below the photosphere is about $300 \mathrm{~km}$, but for numerical reasons, this scale height is stretched in our boundary condition to about $1000 \mathrm{~km}$. On the other hand, for the relatively short distance of about $300 \mathrm{~km}$ that we need to extrapolate into the solar interior, this choice seems to be relatively safe, because the typical length-scales of advectional structures in the upper convection zone are usually larger than $1000 \mathrm{~km}$ in the vertical direction. Furthermore, a small error in the horizontal field component at the photospheric level would have next to no influence on our model corona, because mainly the vertical component is relevant for the coronal magnetic field configuration.

The photospheric vertical magnetic field component is given by observations (c.f. Fig. 3.1) that need to be aligned and denoised. A method for denoising observed unaligned images is applied to solar observations and analyzed in Bourdin (2011). In the boundary layer, we restore the observed target magnetogram, interpolated in time, by altering the horizontal components of the vector potential $\vec{A}$ so that the 


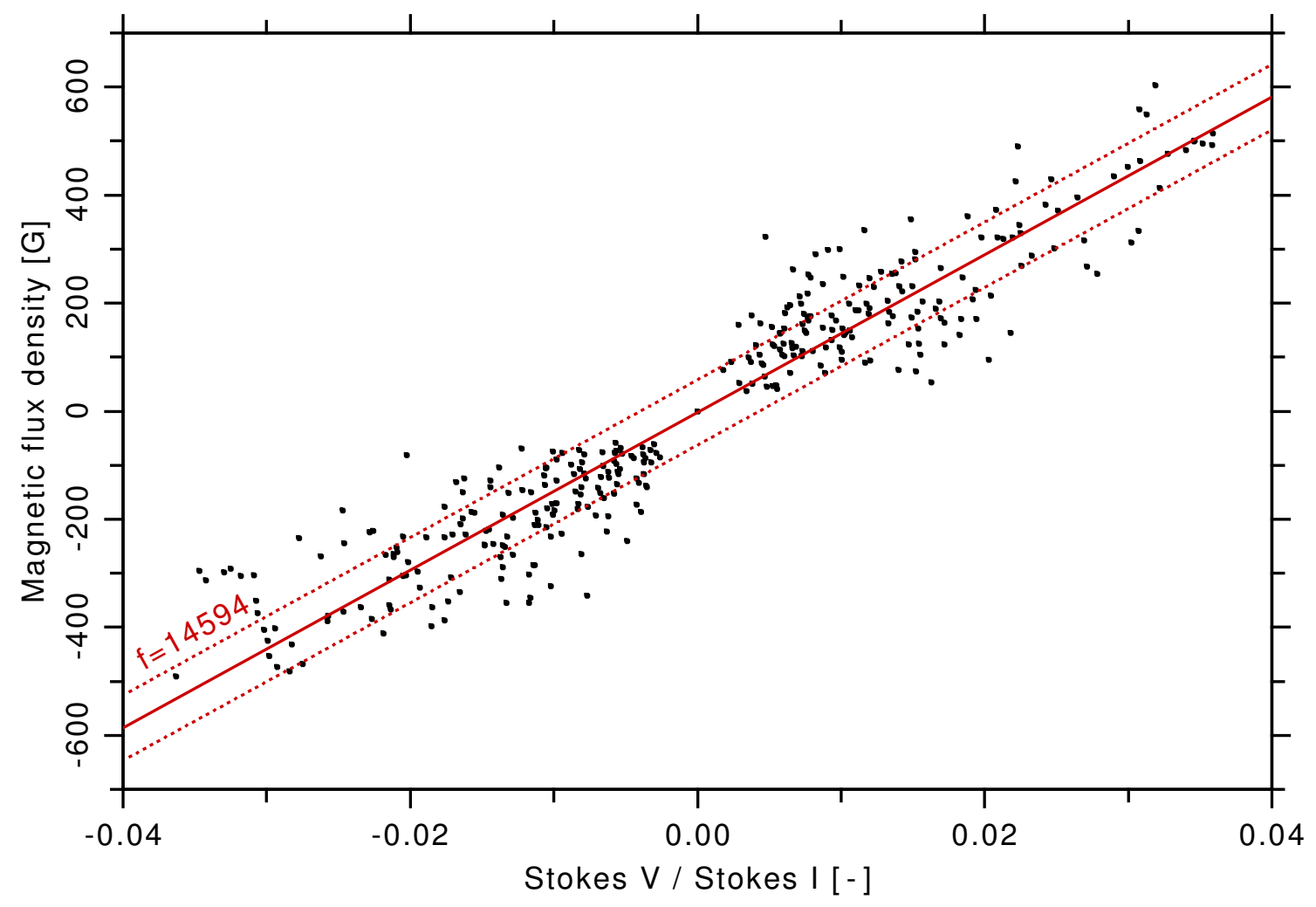

Figure 2.4: Calibration scatterplot relating the linear LOS polarization signal (Stokes $\mathrm{V}$ ) and the intensity (Stokes I) to the magnetic flux density of a spectro-polarimetric reconstruction in a small area in the center of the AR. The red solid line is a LAD fit through the data points and the dotted line indicates the mean absolute deviation. See Sect. 2.3.4.1.

vertical magnetic field is slowly pushed into the observed state with a half-time of $1000 \mathrm{~s}$. This time scale is longer than the life-time of a granule, so that this mechanism won't inhibit the shuffling motions to advect the field-line footpoints. For the calibration of the line-of-sight (LOS) magnetograms, that are provided only as polarization signal, we use a spectro-polarimetric reconstruction of vector magnetograms that are provided only for a small central area between the two main polarities of the AR. In Fig. 2.4 we show the calibration scatter plot together with a least-absolute-deviation (LAD) fit of the data. We get a calibration factor of $f=14594 \pm 276 \mathrm{G}$ to convert the high-cadence linear polarization and intensity data into maps of LOS magnetic flux density in G.

Further information on how we deduce the driving motions in the photosphere from the magnetogram time series is given in Sect. 3.3.2. 



\section{Observationally driven model of the corona above an AR}

* This chapter is published as a journal article (Bourdin et al. 2013 A\&A 555, A123).

Aims: The goal is to employ a 3D magnetohydrodynamics (MHD) model including spectral synthesis to model the corona in an observed solar active region. This will allow us to judge the merits of the coronal heating mechanism built into the 3D model.

Methods: Photospheric observations of the magnetic field and horizontal velocities in an active region are used to drive our coronal simulation from the bottom. The currents induced by this heat the corona through Ohmic dissipation. Heat conduction redistributes the energy that is lost in the end through optically thin radiation. Based on the MHD model, we synthesized profiles of coronal emission lines which can be directly compared to actual coronal observations of the very same active region.

Results: In the synthesized model data we find hot coronal loops which host siphon flows or which expand and lose mass through draining. These synthesized loops are at the same location as and show similar dynamics in terms of Doppler shifts to the observed structures. This match is shown through a comparison with Hinode data as well as with 3D stereoscopic reconstructions of data from STEREO.

Conclusions: The considerable match to the actual observations shows that the field-line braiding mechanism leading to the energy input in our corona provides the proper distribution of heat input in space and time. From this we conclude that in an active region the field-line braiding is the dominant heating process, at least at the spatial scales available to current observations. 


\subsection{Motivation}

Many processes have been identified that are able to deliver a sufficient amount of energy at the base of the corona to heat the plasma to more than $10^{6} \mathrm{~K}$ (e.g., Klimchuk 2006: Mclntosh et al. 2011; Wedemeyer-Böhm et al. 2012). One of them is Ohmic dissipation of currents that are induced by the braiding of magnetic field lines rooted in the photosphere (Parker 1972), which we use in this work. Recently, Cirtain et al. (2013) claimed to have directly observed this braiding. The goal of the present study is to investigate the coronal structure and dynamics resulting from this process by means of a forward model. We synthesize emission line profiles from a numerical 3D magnetohydrodynamics (MHD) model that allows a direct comparison to actual observations. This provides a crucial test for the distribution of the heat input in space and time through the field-line braiding process.

Previous studies modeled the global magnetic structure of the Sun and reproduced actual observations with a prescribed coronal heating function (e.g., Lionello et al. 2005). The first proper implementation of Parker's field-line braiding process was achieved by Gudiksen and Nordlund (2002 2005a b). To fit the active region into the computational box, they had to downscale the domains side-length by a factor of five. This reduces the total magnetic flux and, more importantly, this eliminates the magnetic-field patches of the network by averaging in space. Thus, the magnetic connections from the core of the active region to the surrounding network are not included. Nonetheless, with this model Gudiksen and Nordlund (2002 2005a b) found a loop-dominated corona, where synthesized emission line profiles reproduced observations in a statistical sense (Peter et al. 2004 2006), in particular concerning the persistent redshifts in the transition region (Peter and Judge 1999; Peter 1999). In these and in later studies (c.f. Sect. 3.3.1) the comparison to observations is done statistically or by comparing typical structures. These models were not compared directly with observations by matching the magnetic field at the lower boundary in the photosphere and at the same time reproducing the observed coronal emission.

In this new study we aim at a one-to-one comparison between a 3D MHD model and observations to test the field-line braiding mechanism. For the first time we have done this for a full active region $\alpha$ the correct spatial scale in a large numerical experiment matching the observable spatial resolution. Therefore, this is a major step towards a realistic description of the corona in a 3D model.

While the 3D model cannot resolve the actual dissipation length scales that go down to the meter scale and below, it does provide a self-consistent treatment of the energy input, redistribution, and radiative losses to get a proper coronal energy 
balance. This redistribution of energy, in particular the heat conduction along the magnetic field, is essential to self-consistently set the coronal plasma pressure, which is a prerequisite when synthesizing coronal emission that is to be compared to actual observations. Because of the limitations of the spatial resolution our model as well as previous 3D MHD models do not resolve the individual nanoflare reconnection events proposed by Parker (1988). The process actually described in the numerical models might be better characterized as magnetic diffusion.

We first discuss the general model strategy (Sect. 3.2) before giving some details on the model setup (Sect. 3.3) and presenting our results (Sect. 3.4 and 3.5).

\subsection{Model strategy}

The central idea behind this study is to compare synthesized emission from a forward 3D MHD coronal model driven by photospheric observations to actual coronal observations. For this we use observations of the magnetic field and horizontal velocities in the photosphere to prescribe the lower boundary of the 3D MHD model. From the model we synthesize emission line spectra which are observable with current extreme UV spectrographs, and are thus directly comparable to coronal observations.

For our study we select an active region (AR) for which observations have been taken simultaneously in the photosphere and in the corona (Fig. 3.1). We use a data set from the Hinode solar space observatory (Kosugi et al. 2007), which includes observations from the X-ray telescope XRT, spectra of Fe XII and Fe XV from the extreme UV imaging spectrometer (EIS, Culhane et al. 2007), and the spectro-polarimeter (SP) and narrowband filter imager (NFI) of the solar optical telescope (SOT, Tsuneta et al. 2008). The SP and the NFI provide vector- and line-of-sight magnetograms, and horizontal velocities in the photosphere.

The active region under investigation did not show sunspots, but a set of hot loops is visible in X-rays (Fig. 3.1 a). These connect two extended regions of strong magnetic field with opposite polarity (Fig. 3.13). We use a time series of magnetograms to define the magnetic field and horizontal velocities at the bottom boundary of the computational domain (see Sect. 3.3.2).

Data from Hinode/EIS provide a raster map of the active region, including the Fe XII (195 $\AA$ ) and Fe XV (284 $\AA$ ) emission lines. From these we derive the intensity and Doppler shifts (Fig. 3.2 a,b). After a careful spatial alignment (Sect. 3.3.3) we can then compare the coronal observations to the synthetic model data (Sect. 3.4).

The coronal model is powered by the observed photospheric magnetic field that is 

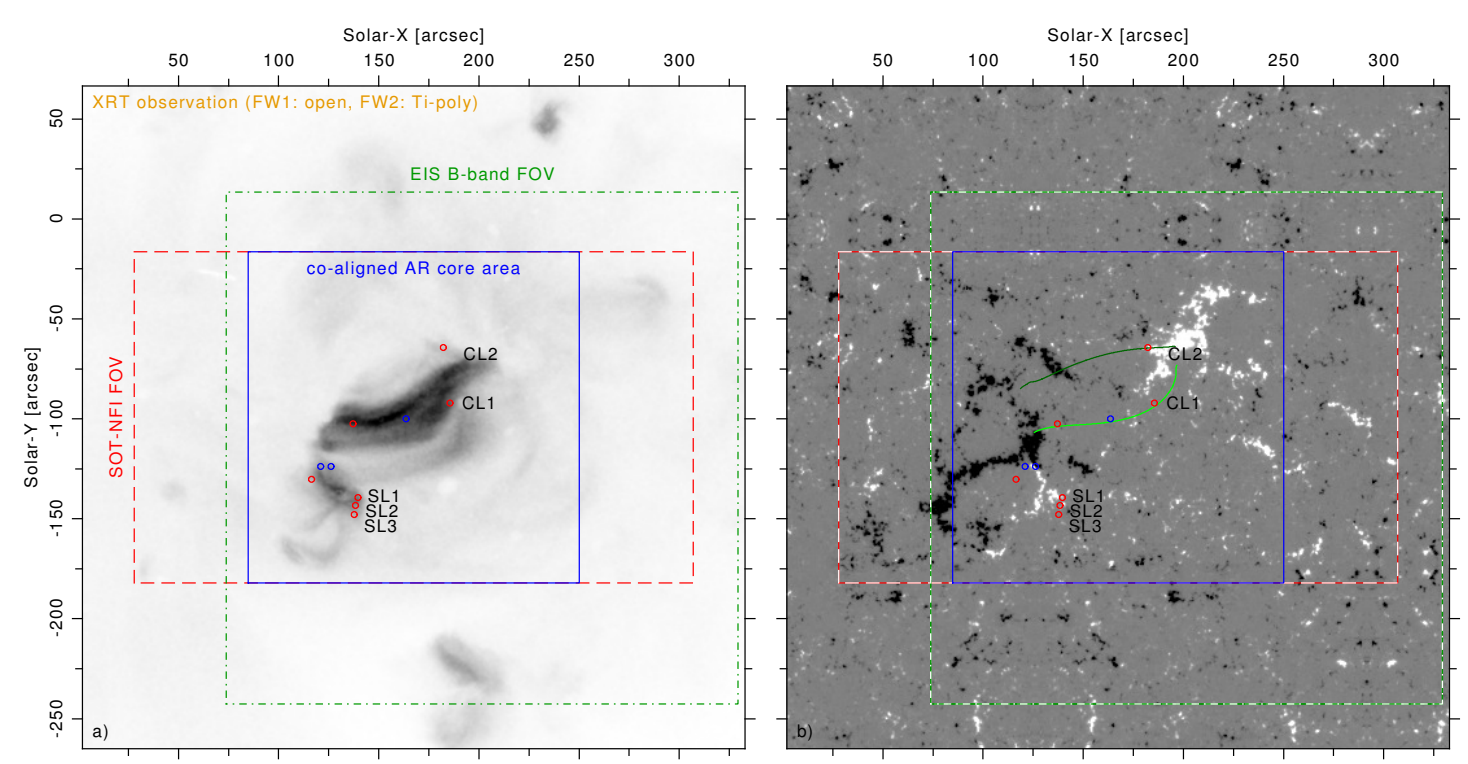

Figure 3.1: Active region observed by the Hinode satellite on 14 Nov 2007. The left panel shows the X-ray emission observed by XRT together with the field-of-view of EIS (green dash-dotted square) and SOT-NFI (red dashed rectangle). The right panel displays a line-of-sight magnetogram (saturation level: $\pm 300 \mathrm{G}$ ) of the active region that is smoothly embedded in a quiet Sun carpet (see Sect. 3.3.2). We use only the co-aligned AR core area (blue solid square) for our analyses. The circles and lines indicating various loop structures are co-spatial with those in Fig. 3.2.

advected by the observed photospheric horizontal velocities. This leads to field-line braiding and induces currents in the corona that are dissipated and heat the plasma. The 3D MHD model provides the temperature, density, and velocity at each grid point of the computational domain. Following the approach of Peter et al. (2004 2006) we use the atomic data base CHIANTI (Dere et al. 1997; Young et al. 2003) to synthesize emission lines. This provides maps of intensity and Doppler shift that can be compared directly to the coronal observations.

This strategy enables us to test our model and the underlying theoretical assumptions for the coronal energy input, i.e., braiding of magnetic field lines and the subsequent Ohmic dissipation of induced currents. The aim is to check if the model description is sufficient to reproduce realistic coronal structures and their dynamics. 


\subsection{MHD model and alignment with observations}

\subsubsection{Coronal model}

The basic setup of our numerical experiments follows the philosophy of Gudiksen and Nordlund (2002 2005a b) and Bingert and Peter (2011). The initial condition of our model consists of a stratified atmosphere in hydrostatic equilibrium. We initialize the magnetic field configuration with a potential field extrapolation from the observed photospheric magnetogram.

For the temporal evolution of the model we employ the compressible resistive MHD equations to compute the temperature, velocity, density, and the magnetic vector potential inside the computational domain. The photospheric driving advects the magnetic field, which induces currents $j$ in the upper atmosphere that lead to Ohmic heating $\mu_{0} \eta j^{2}$. For the magnetic diffusivity $\eta$ and also for the kinematic viscosity we use a constant value of $10^{10} \mathrm{~m}^{2} / \mathrm{s}$ in the corona. For details of the model the reader is referred to Bingert and Peter (2011).

As outlined by Bingert and Peter (2011) we choose $\eta$ so that the current sheets that form have a finite width still resolved by our numerical scheme; in other words, we choose $\eta$ so that the magnetic Reynolds number is of the order of unity when choosing the grid spacing as a length scale. Therefore our heating term $\mu_{0} \eta j^{2}$ should be considered a parameterization of the true heating mechanism. A full model, including the actual dissipation process and covering a macroscopic structure observable on the Sun (e.g. a whole active region) is beyond current capabilities. Some steps of models in the context of solar flares going beyond the MHD picture including kinetic processes, for example, can be found in Cargill et al. (2012). In contrast to our approach, Lionello et al. (2005) use a prescribed heating function in their 3DMHD model. There magnetic energy is dissipated through an explicit term in the equations or by numerical diffusion, but this is not consistent with the chosen heating function. In our approach, the magnetic energy actually dissipated (in the induction equation) is converted into heat (through $\mu_{0} \eta j^{2}$ in the energy equation). We consider our treatment to be more consistent than simply prescribing a heating function.

Our model includes gravity, heat conduction parallel to the magnetic field following Spitzer (1962), and optically thin radiative losses based on Cook et al. (1989).

The heat conduction is of pivotal importance because it sets the pressure in the corona, and thus is essential if one wants to compare the synthesized emission to actual observations.

Summing up, we have a self-consistent description of the thermal structure of the plasma and of the magnetic field in the coronal structures. With a spatial resolution 
of down to $100 \mathrm{~km}$ this 3D MHD model cannot match the resolution possible in ID loop models, of course. Still, the implementation of the field-aligned heat conduction together with the optically thin radiative losses allows us to properly describe the energy cycle between the chromosphere and the corona. Here the Ohmic heating of the corona leads to heat conduction back to the chromosphere, which together with the local heat input there leads to evaporation of material that then expands into the corona. The description of this cycle is important because it basically sets the pressure of the coronal structure (Withbroe 1988). With the limits of the spatial resolution in a 3D model the temperature gradients are less steep than in a ID loop model, but still the energy cycle between the chromosphere and corona is accounted for.

Models similar to the one presented here were able to produce a loop-dominated corona (Gudiksen and Nordlund 2002 2005a|b) where synthesized average quantities matched observables such as the differential emission measure and the transition region Doppler shifts (Peter et al. 2004 2006; Hansteen et al. 2010; Zacharias et al. 2011a). Furthermore, these models provided a new way to understand loops with constant cross section (Peter and Bingert 2012) and provided insight in the spatio-temporal distribution of the heat input into the corona (Bingert and Peter 2011, 2013).

To run the numerical experiments, we use the Pencil Code (Brandenburg and Dobler 2002 I. The parameters of the simulation are available at the Centre de Données astronomiques de Strasbourg (CDS) ${ }^{2}$. The computational domain covers $235 \times 235 \mathrm{Mm}^{2}$ horizontally and $156 \mathrm{Mm}$ vertically with $1024 \times 1024 \times 256$ grid points. The horizontal grid spacing is $230 \mathrm{~km}$, which is roughly the spatial resolution of the magnetograms employed for the photospheric driving of our model. In the vertical direction we use a non-equidistant grid to resolve the strong gradients in temperature and density with a resolution of about $100 \mathrm{~km}$ up to the transition region.

We advanced the model in total for about 65 minutes solar time. After about 50 minutes, the model reaches a state independent of its initial condition. During the last 15 minutes, strong Ohmic heating sets in and the peak temperature in the box rises from $0.5 \mathrm{MK}$ to about $1.4 \mathrm{MK}$, where the rapid increase comes to a halt. The system reaches a quasi-stationary state and individual structures develop.

\footnotetext{
'http://Pencil-Code.Nordita.org/

${ }^{2}$ http://CDS.U-Strasbg.fr/
} 


\subsubsection{Lower boundary condition from observations}

The observed magnetogram time-series has a cadence of 90 seconds. From that we deduce horizontal motions of the magnetic patches by local correlation tracking. The typical spatial scale of these patches is $15 \mathrm{Mm}$ (about 10 granules) and their velocity distribution peaks at $100 \mathrm{~m} / \mathrm{s}$. With this method the solar granulation on a scale of $1 \mathrm{Mm}$ remains unresolved. Therefore, we generate a horizontal velocity field by using a method described in (Gudiksen and Nordlund 2002) that matches statistical properties of observed granulation and that we have used before (e.g., Bingert and Peter 2011). The velocity field we use as a driver in our simulation is the superposition of the observed flow field and the generated field on smaller scales.

The FOV of the NFI data covers just the active region magnetic field concentration (see Fig. 3.1p). This FOV is not large enough for our simulation and would cause problems with the side boundary conditions, which are in our case periodic. Therefore, we smoothly embed the observed $A R$ inside a periodic carpet of mirrored quiet Sun (QS) magnetogram patches that we also took from observations (see Fig. 3.1 ). In this process we ensure that the magnetogram at the bottom is periodic. This additional QS area isolates the main magnetic patches in the periodic setup. This ensures a more realistic magnetic field topology and allows field lines to connect from the main polarities into the QS network. For the calibration of the magnetograms we use several snapshots of the AR core that are available as spectro-polarimetric SOT/SP level-1 data. This procedure provides a magnetogram time-series that we interpolate in time to update the lower boundary during the simulation.

The magnetogram time-series together with both large- and small-scale velocity fields prescribe the lower boundary of our model. This drives our simulation from the bottom by shifting the footpoints of the magnetic field lines, a process often called braiding. Through this a net upward Poynting flux carries energy into the corona. Induced currents lead to heating in the corona by Ohmic dissipation, be it through field-line braiding (Parker 1972) or through current sheets formed by coronal tectonics (Priest et al. 2002).

\subsubsection{Alignment between observations and simulation}

To compare the observations with our simulation results, we need to align the observations spatially, in particular the magnetogram that drives our model and the EIS raster maps which we want to compare with the synthesized coronal emission. We use the magnetogram in the middle of the time series as a reference and 
align all magnetograms to it. By this we can correct for solar rotation as well as for the proper motion of the AR. To align these photospheric magnetograms with coronal observations, we first align co-temporal snapshots of NFI magnetograms with chromospheric Hell emission recorded by EIS (B-band). Because Fe XII is recorded on the A-band, we also have to correct for the constant spatial shift between the two EIS bands (Kamio et al. 2010). We do this by aligning maps in Si VII (A-band) and Fe VIII (B-band), which form at similar temperatures. As a final step, we align the map in Fe XV recorded by EIS to the $X$-ray maps taken by XRT with the Ti-poly filter.

Most of the aligned images differ slightly in shape and contrast, and we estimate the alignment in each step to be accurate within about 1.5 arcsec. The spatial sampling of EIS and XRT are 1 and 2 arcsec, respectively. Because the alignment between any two instruments consists of several steps, and considering the correction for rotation and the AR proper motion, we estimate the overall accuracy to be about 3 arcsec. Based on the loop footpoints of the short loop system (SL 1-3 in Fig. 3.2) we find an alignment residual of 3.5 arcsec towards north-west. We subtracted it to make Fig. 3.2 clearer. The circles in Fig. 3.2 have a diameter of 3 arcsec, indicating the accuracy of the alignment.

\subsection{Hot loops in the core of the active region}

Based on the temperatures, densities and velocities in the 3D MHD model we synthesize profiles of coronal emission lines observable with EIS (following Peter et al. 2006). In Fig. 3.2 we show the observations (left column) and the synthesized maps (right column). In both observation and synthesized model data we can identify a system of short loops (SL 1-3) and longer core loops (CL 1, 2) in the active region core.

The short loops are identifiable as separate loops in both the observed and synthesized Fe XV emission - their length, width, and footpoint location coincides within the given accuracy of the alignment. Loops SL 1 and 2 are brighter than SL 3 in both synthetic emission and the observation, so that our model matches the observation of this short loop system very well. The different curvature of these short loops could indicate a projection effect in the observation due to an inclination of the loops. For the longer loops there is good agreement between observation and model in the position and shape of CL 1, even though the synthetic emission is not as strong as the observed emission. A much weaker (and cooler) loop CL 2 can only be identified in the model data. Both CL 1 and 2 are visible in the simulation only since a few solar minutes. 


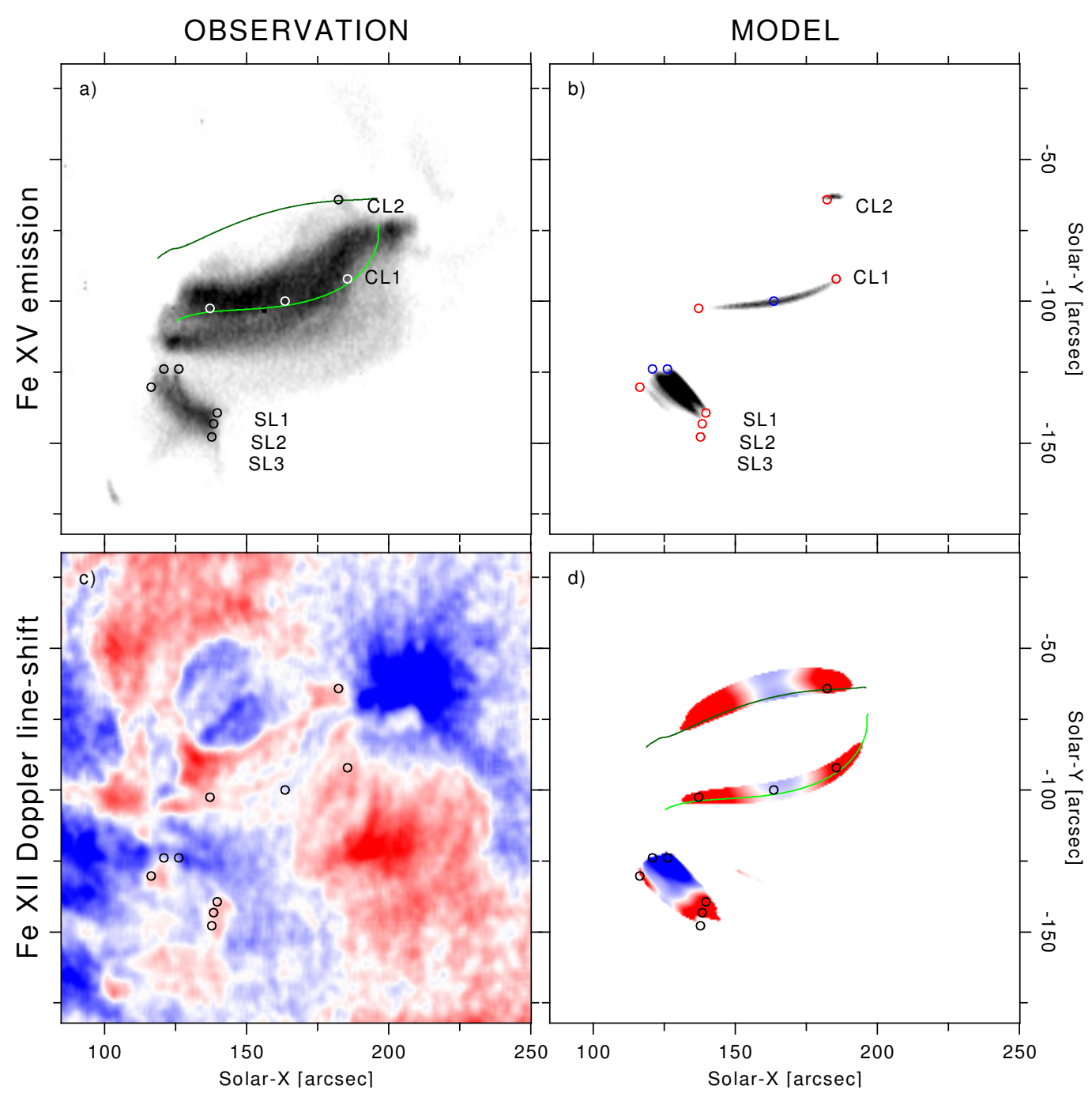

Figure 3.2: Direct comparison between observations and the 3DMHD forward model. The left column shows the active region as observed by EIS on 14 Nov. 2007. Panel(a) displays the intensity map in FeXV (284 $)$ on a linear inverse scale normalized to the peak intensity. For plotting we set a threshold of $1 / 6$ of the peak intensity, which is well above the noise level. In panel(c) we plot the Doppler map in Fe XII $(195 \AA)$. The scale of the doppler map covers $\pm 10 \mathrm{~km} / \mathrm{s}$, where blue-shifts indicate plasma flows towards the observer. The right column shows the corresponding quantities synthesized from the 3D MHD model with the same color coding. A short loop system can be seen spanning from one of the two main polarities to the network of the quiet Sun (SL 1-3), as well as a loop (system) in the core of the AR between the two main polarities (CL 1). In the model we traced two magnetic field lines, rooted in the centers of $C L 1$ and 2, that are overplotted in green. The circles are located at the same positions in all panels. The alignment between the observations and synthesized images is accurate within about 3 arcsec corresponding to the diameter of the circles. 
The temperature of CL 1 is still rising, which indicates that the heating in the system of field lines around CL 1 is getting stronger. Therefore, we expect CL 1 to develop into a brighter and broader structure in time. This should improve the similarity of the synthesized emission to the observation.

From this comparison of the observed and synthesized emission we can conclude that the model has a distribution of the energy input in space and time to create the actually observed structures. We now turn to the flows resulting from the dynamics in the active region.

To investigate the dynamics in the $A R$, we compare the observed line-of-sight integrated Doppler line-shifts with the synthetic ones (bottom row in Fig. 3.2). Here we use the FeXII line, because in the observation this line provides a clearer, less noisy Doppler map than does Fe XV. The synthesized Doppler maps in Fe XII and Fe XV are quite similar, however. In general, the synthetic Doppler shifts along the hot loops in the corona correspond well to their observed counterparts (Fig. 3.2 k,d). The northern footpoints of the short loops SL 1 and 2 are located in a region with magnetic cancellation (see Fig. 3.1P) and so experience increased heating. Here we find upflows (blue-shifts). The resulting siphon-flows along SL 1 and 2 towards the southern footpoints are driven by the asymmetric heating. Rooted farther away from the flux cancellation region we also see a cooler loop (SL 3) with material draining all along the loop as a result of cooling.

In the AR core we see downflows in both loop legs of CL 1 together with a rising loop-top. This is consistent with an emerging loop, where plasma is pushed up (blueshift at apex) and then falls down the legs (redshift at footpoints). The synthetic Doppler shift at the loop-top corresponds to a vertical velocity of about $2 \mathrm{~km} / \mathrm{s}$, which was also deduced from observations of young loops in an emerging AR (e.g., Solanki et al. 2003).

\subsection{STEREO 3D reconstruction}

Besides the Hinode observations, the investigated AR was also observed simultaneously by the STEREO satellites, which allows us to reconstruct the $3 \mathrm{D}$ shape of the coronal loops. At the observation time, the two satellites had a viewing angle of $40^{\circ}$ between them. We traced the Fe XV intensity structures observed in the $284 \AA$ channel of STEREO $A$ and $B$ by first locating both loop legs, then the loop-top in the middle, and finally determining co-spatial points inbetween. We used the function 'scc_measure' version 1.15 availabe in the SolarSoft library ${ }^{3}$. The reconstruction is accurate to several pixels, corresponding to

\footnotetext{
${ }^{3}$ http://www.Imsal.com/solarsoft/
} 

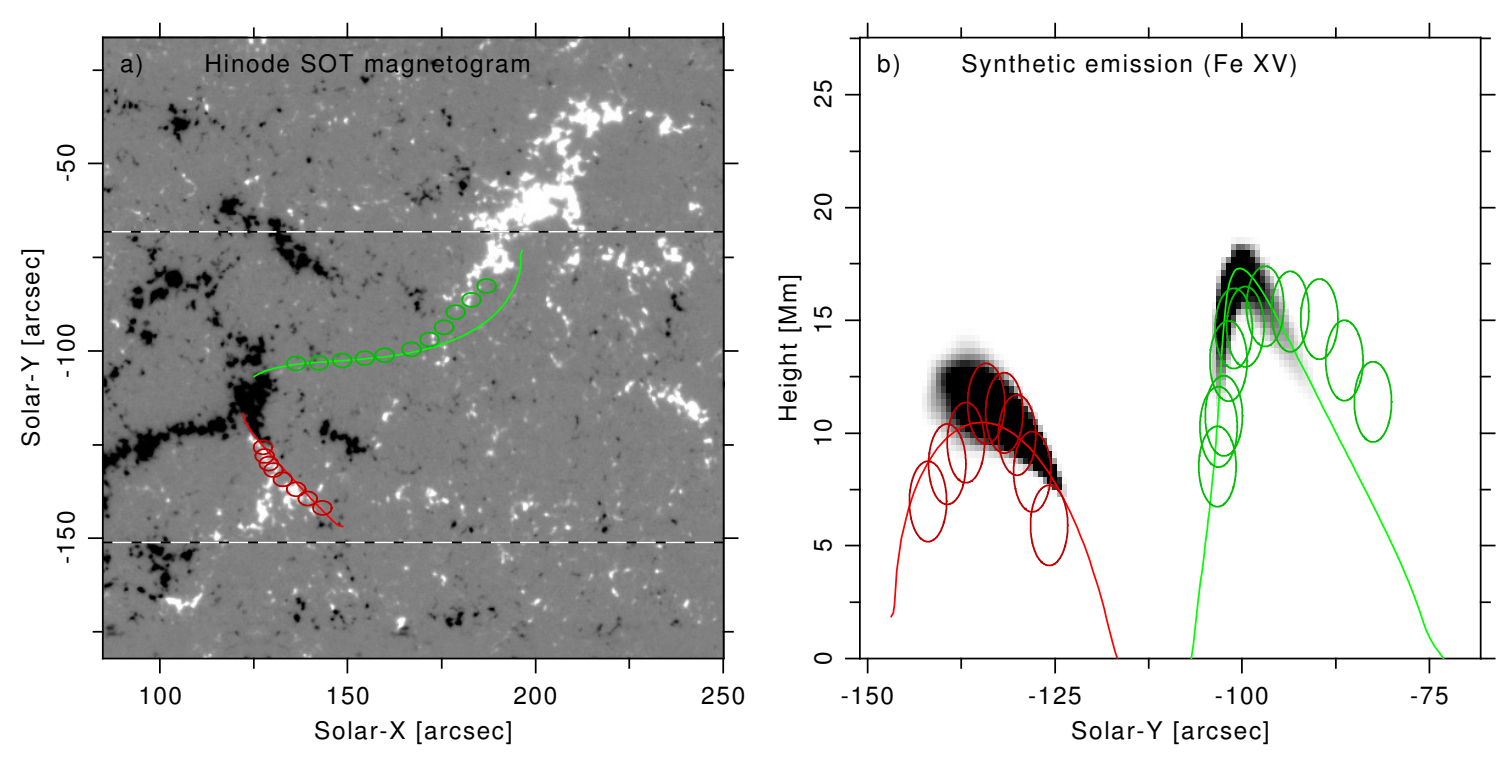

Figure 3.3: Direct comparison of the model intensity structures with the STEREO 3 D reconstruction. Panel (a) displays the magnetogram (saturated at $\pm 300 \mathrm{G}$ ) at the bottom boundary. Overplotted are the projections of those field lines from the model that cross the maximum of the synthesized emission of the respective loop in the 3D computational domain. The circles show the projection of the loops reconstructed from the STEREO observations. The diameter of the circles indicates the uncertainty in the reconstruction. In panel(b) we show the synthesized model intensity in Fe XV (284 $\AA$ ) as seen from solar east, i.e., along solar-X, again with the 3D reconstruction. The colors denote the core loop (CL 1, green) and the short loop (SL 1, red) as introduced in Fig. 3.2 see Sect. 3.4. The dashed white lines in panel(a) indicate the range of solar-Y displayed in panel (b).

the width of the traced structures that we estimate to about 5 arcsec, resulting in uncertainties of several $\mathrm{Mm}$.

A comparison of the loop 3D coordinates reconstructed from the STEREO observations with the loops synthesized from the model is shown in Fig. 3.3. For this we plot the magnetic field line that passes through the point of maximum emission of the synthesized loop in the 3D domain for one of the core loops (CL 1) and one of the short loops (SL 1). In the left panel of Fig. 3.3 we show the view from the top (along with the magnetogram at the bottom boundary as the background), and in the right panel we plot the projection of the field lines when looking at the computational domain from the side, now together with the synthesized emission of the loops integrated horizontally through the box (along solar-X). The reconstructed 3D coordinates of the loops observed by STEREO are overplotted as a sequence of circles, the diameter of the circles indicating the 
uncertainty of reconstructed position. We find that the synthetic emission from loops CL 1 and SL 1 are located within the 3D reconstruction, both in the horizontal and in the vertical direction. Most importantly, the reconstructed loops also reach similar heights as the synthesized model loops. This implies that the model (in general) reproduces the observations also in its $3 \mathrm{D}$ structure. In the observation we still find a slight inclination of the SL 1 loop that is not found in the model.

\subsection{Conclusions}

We have presented a 3D MHD model of the corona that is driven by observations of the solar photosphere. The synthesized profiles of coronal emission lines show strong similarities to the actual observations of the some region on the Sun. This applies to the line intensities and the Doppler shifts that reflect the dynamics within the coronal loops. Even the spatial distribution of the synthetic emission within the $3 \mathrm{D}$ computational domain occurs roughly at the same location as reconstructed from stereoscopic observations.

In our model all coronal loops examined are heated predominantly by Ohmic heating, which is induced by the braiding of field lines through the (horizontal) photospheric motions. The average Poynting flux into our model corona roughly matches the predicted value of about $300 \mathrm{~W} / \mathrm{m}^{2}$. Other processes, such as viscous heating of material draining from the corona, also play a role, albeit not the dominant one. Because the hot structures in the simulation develop at the same locations found in observations, we conclude that the heat in the simulation is deposited in the same places as on the real Sun. The (asymmetric) heating of the loops and the rise of magnetic field lines leads to flows in the loops that are, again, similar to those found in the observation.

This good match is found even though we cannot resolve the small scales on which the actual dissipation of magnetic energy occurs. At least the energy deposition at scales accessible to our model, as well as to current coronal observations (above about $500 \mathrm{~km}$ ), is well represented by our proxy for the Ohmic heating $\mu_{0} \eta j^{2}$. Certainly, on smaller scales many other processes will operate, and we conclude that a heating proportional to the square of the currents provides a good proxy for these sub-grid processes.

The substantial match to the observation shows that the field-line braiding originally proposed by Parker (1972) provides sufficient energy with the proper distribution in space and time to reproduce characteristic features such as hot coronal loops and their dynamics in an active region. 


\section{Coronal energy input and dissipation}

* This chapter is based on a journal article that is in preparation.

\subsection{Motivation}

Since we have a 3D MHD model that shows coronal loops matching to the ones of an observed $A R$, and from the model we can deduce practically all physical quantities like the magnetic field, the plasma pressure, and the plasma motions, we practically have all ingredients at hand that were necessary to create those hot loops in the AR core. We will first have a look at large-scale quantities, like the energy flows in the model (Sect. 4.2) and then do statistical analyses on samples of individual field lines (Sect. 4.3). Our goal is to understand, which quantities relate in which way to the coronal heat input and temperature.

\subsection{Energy input viewed at large}

\subsubsection{Overview of whole box and subvolumes}

To investigate the spatial distribution of the energy input into the model corona, we look at the individual heating terms in a horizontal average at a given height. The driving in the bottom boundary layer provides an energy input by Poynting flux into the model.

\subsubsection{Contribution to the internal energy}

In Fig. 4.1 we show which energy dissipation mechanism is active in which height for different fields of view (FOV). While below the corona all profiles lie relatively close together, looking at the full FOV data (dashed lines) we see that in the corona the Ohmic heating dominates over the viscous heating in almost all heights. 


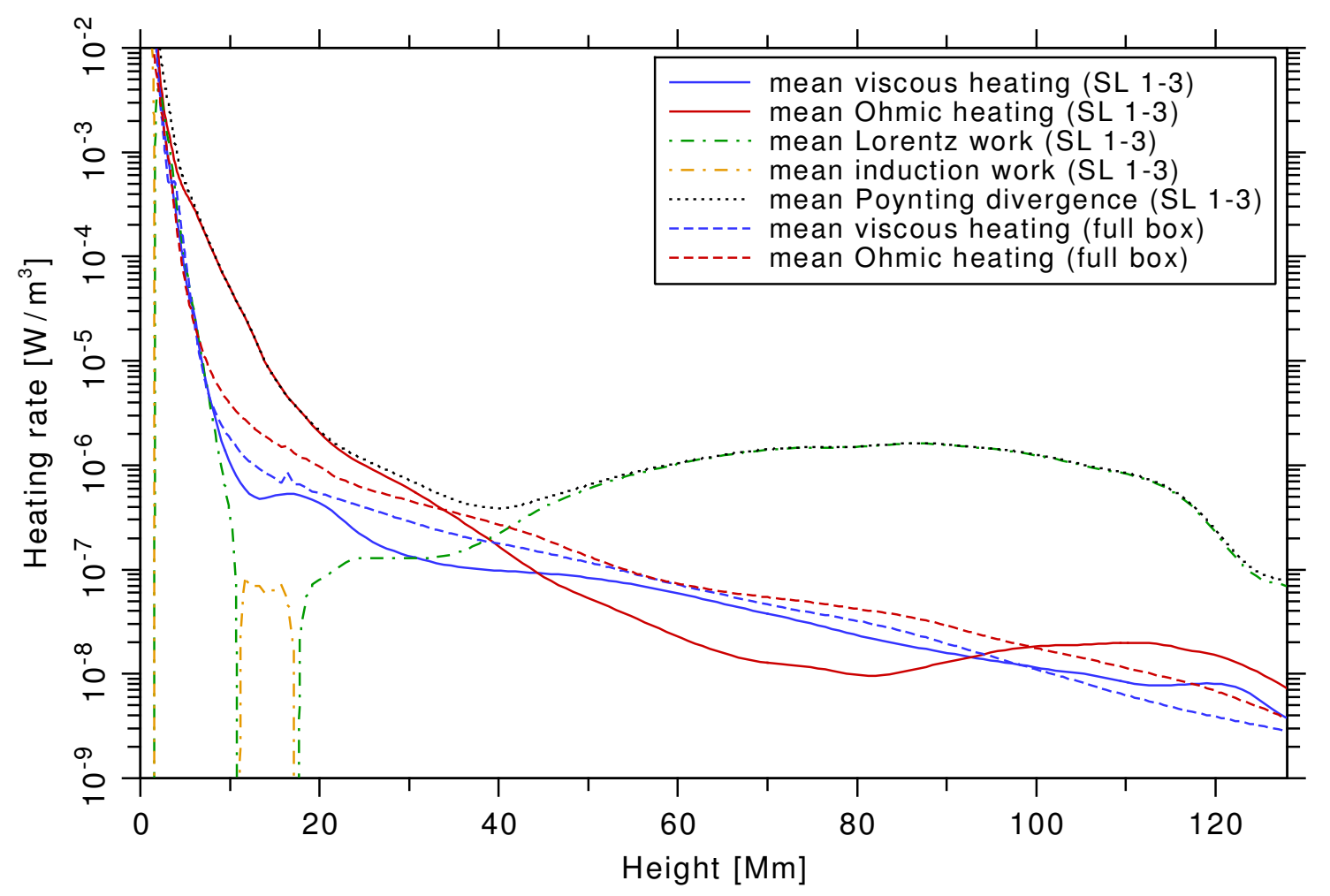

Figure 4.1: Horizontal averages of the volumetric Ohmic (red) and viscous heating (blue) plotted as a height profile. The line styles depict the different FOVs given in brackets in the legend. The dash-dotted lines basically describe the same physical quantity $W_{\text {Lorentz }}=\vec{u} \cdot(\vec{\nabla} \times \vec{B} \times \vec{B}) / \mu$, while the Lorentz work (green) and the induction work (orange) are of opposite sign. The Ohmic heating is significantly smaller above the hot AR core (40 to $90 \mathrm{Mm}$ height, red solid line) in comparison to the full box average (red dashed line), see Sect. 4.2.1.1.

The scale height of these two quantities are very similar and uniform in the whole corona. When we restrict the FOV to the hot AR core loops SL 1-3 (solid lines) the Ohmic heating profile gets more structured. Between the heights of 5 and $30 \mathrm{Mm}$, where the hot loops are located, we see a significant increase of the Ohmic heating as compared to the full FOV.

Also we see a shortage of the Ohmic heating above the hot AR core loops in heights between $40 \mathrm{Mm}$ and $90 \mathrm{Mm}$ in relation to the full FOV average (c.f. Fig. 4.1). This can be understood by assuming that the energy transport is directed mainly upwards and that an increased dissipation in a lower layer would decrease the possible energy input to an upper layer. Of course there is more to consider, e.g. most of the field lines that close well above the hot $A R$ core subvolume are rooted outside that area, so that energy can be transported there while following 
the magnetic field.

Both, the full and reduced FOV profiles in Fig. 4.1 are roughly joining up again at heights above $90 \mathrm{Mm}$, which indicates that either some energy is transported to above the hot AR core loops subvolume from outside. Or alternatively, this high up part of the corona could simply not yet fully become independent of the initial condition, because the Alfvén travel time for magnetic perturbations from the bottom layer into the upper corona is significantly larger than into the hot $A R$ core loops system.

The profile of the divergence of the Poynting flux (black dotted) mainly follows the sum of the Ohmic heating and the Lorentz work (green dash-dotted in Fig. 4.1). Between 10 and $20 \mathrm{Mm}$ we find a region that is in average dominated by induction work (orange dash-dotted). For the full FOV average, this induction region would expand farther from 10 to about $55 \mathrm{Mm}$. The viscous heating also shows deviations between the two FOV averages, but the difference is much smaller than the deviations seen in the Ohmic heating (red and blue lines in Fig. 4.1). Also for the smaller FOV, the viscous heating is smaller between heights of about 10 to $100 \mathrm{Mm}$ as compared to the full FOV. Altogether, this shows that the heat input to the hot AR core loops in our model is largely dominated by the Ohmic heating.

\subsubsection{Contribution to the temperature}

The question remains, whether the viscous heating has a significant impact on the overall temperature stratification. To address this question, one must also consider the plasma density at the places where the heating occurs in order to understand in how far this volumetric heating can cause an increase in the local temperature. This we do in Fig. 4.2 by looking at the Ohmic and viscous heating rates per particle. We find that the mean Ohmic heating per particle (red) dominates over the viscous heating (blue) already for the full FOV (dashed lines) from a height of $8 \mathrm{Mm}$ upwards.

When restricting the FOV to the hot $A R$ core loops SL 1-3 (solid lines in Fig. 4.2), we see a significant increase of the Ohmic heating per particle in the heights between 5 and $40 \mathrm{Mm}$, where these loops are located. We also see a slight increase of the viscous heating per particle in these heights, but in the upper corona the viscous heating per particle remains roughly unchanged. Still, above the smaller hot AR core FOV, the Ohmic heating clearly dominates over the viscous heating by a factor of 10 and more up to a height of roughly $30 \mathrm{Mm}$. Furthermore, in this region the mean of the Ohmic heating per particle (red solid line) also reaches over any local maximum of the viscous heating (blue dotted line). In the upper 


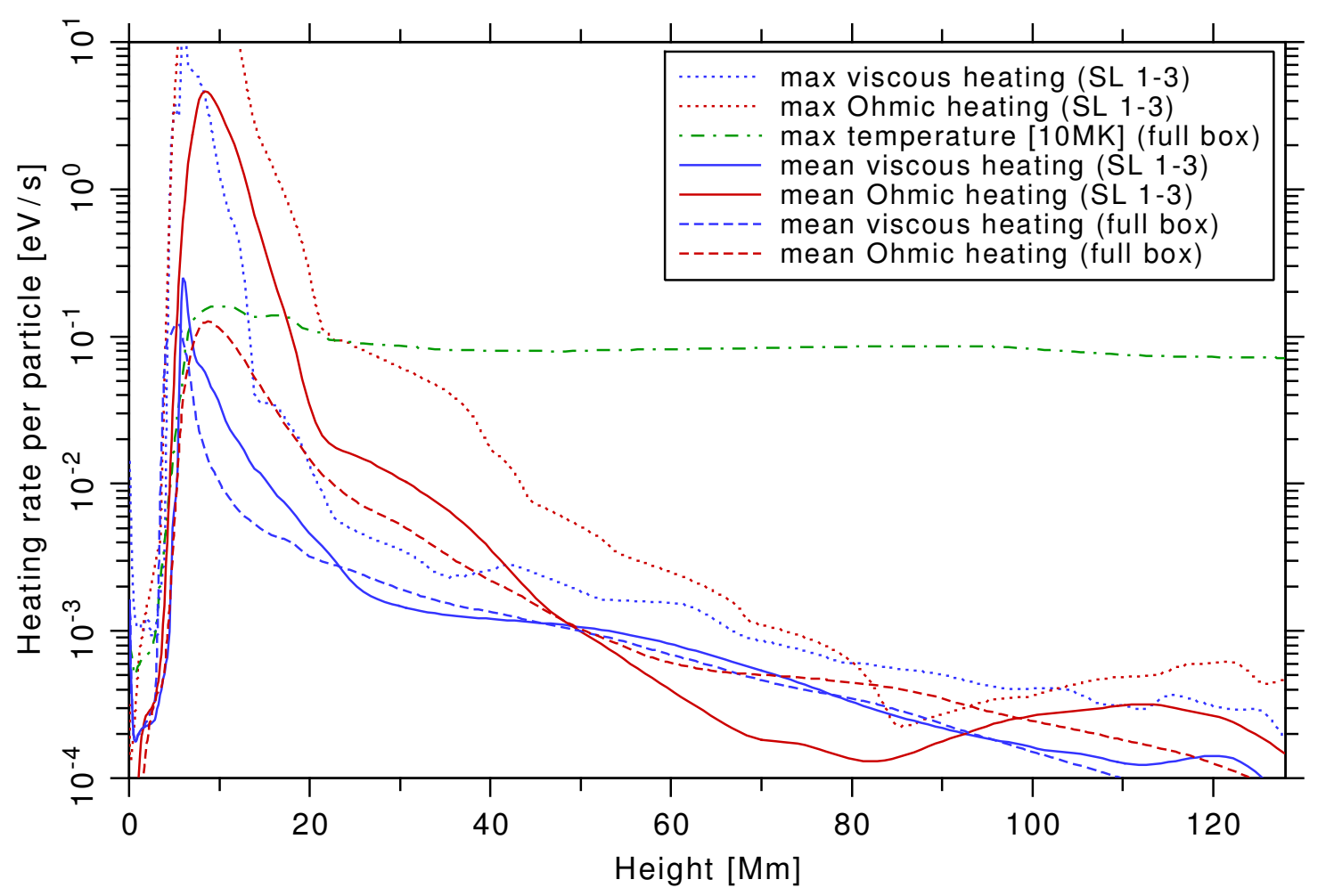

Figure 4.2: Horizontal averages of Ohmic (red) and viscous heating per particle (blue) versus height. The dotted curves indicate the maximum values at each height, while the solid lines show the corresponding mean value. Overplotted is the height profile of the maximum temperature (green dash-dotted). The different FOVs are symbolized as line styles and are given in brackets in the legend.

corona above $50 \mathrm{Mm}$, we see the same tendency as in Fig. 4.1 namely that the Ohmic heating per particle significantly drops below the viscous heating for the restricted FOVs, while this is not the case for the full FOV (dashed lines). The viscous heating can gain dominance over the Ohmic heating above hot closed-field regions that are strongly heated by Ohmic dissipation. And we see that the viscous heating is slightly increased above the crossing point of Ohmic and viscous heating compared to the full box FOV, while it is slightly decreased below this crossing point. In contrast to the restricted FOV, the global mean viscous heating profile has a rather constant scale height above $20 \mathrm{Mm}$. This behavior of the viscous heating could be explained by downflows from the upper corona that are getting disturbed by the magnetically closed AR core. Above $80 \mathrm{Mm}$ the height profiles of the Ohmic heating per particle dominate again over those of the viscous heating with a factor larger than 2 .

Nonetheless, the maximum temperature stratification stays mainly constant in the 
corona above $20 \mathrm{Mm}$. The rapid rise of this temperature profile coincides exactly with the strong rise in Ohmic and viscous heating in the lower 3-7 Mm. The strong gradient of the temperature ends exactly, where the strong increase of the viscous heating ends and doesn't follow the further rise in the Ohmic heating towards $10 \mathrm{Mm}$.

This overview altogether indicates that the main contribution to the high coronal plasma temperatures is by the Ohmic heating, while the viscous heating seems to contribute mainly in higher density areas, where the local input to the internal energy would not result in such a high temperature rise. Still, the location and shape of the transition region temperature rise seems to be determined by the Ohmic and viscous heating equally, where both of them deliver similar amounts of internal energy.

From our model we know that the viscous heat delivered at the transition region is due to downflows from the corona above. These downflows are caused partly due to cooling material converting potential into kinetic energy. Partly, also some material that was previously accelerated by the Ohmic heat input, leads to an increase in the plasma pressure and hence pushes material due to adiabatic expansion. \Therefore, some of the later viscous heating is caused by the initial Ohmic dissipation.

\subsubsection{Energy introduced into the model}

Perturbations in the magnetic field can travel into the corona with the Alfvén velocity. Depending on the speed of the driving motions, these perturbations will follow the magnetic field as transversal or longitudinal waves that could be dissipated in the solar atmosphere, which is referred to as "AC" heating. If the driver only gradually changes a field line, currents can be induced that compensate these slow perturbations, which can be seen as a diffusional process that belongs to "DC" heating. 2 2

The field-line braiding introduced by our lower boundary creates the Poynting flux that powers the coronal energy input. In the photosphere, the applied horizontal motions shuffle around the footpoints of magnetic field lines with velocities of about $1.242 \mathrm{~km} / \mathrm{s}$. This bends the magnetic field that starts to deviate from the initially force-free state and these perturbations can then propagate with roughly the Alfvén speed along the field lines. As a result, a net upwards directed Poynting flux carries

\footnotetext{
'As we show in Sect. 5.4.1.3 a strongly asymmetric Ohmic heating can even cause siphon flows in a coronal loop.

${ }^{2}$ For a detailed description of different $A C$ and $D C$ heating mechanisms the reader is referred to Aschwanden (2004) and Klimchuk (2006).
} 


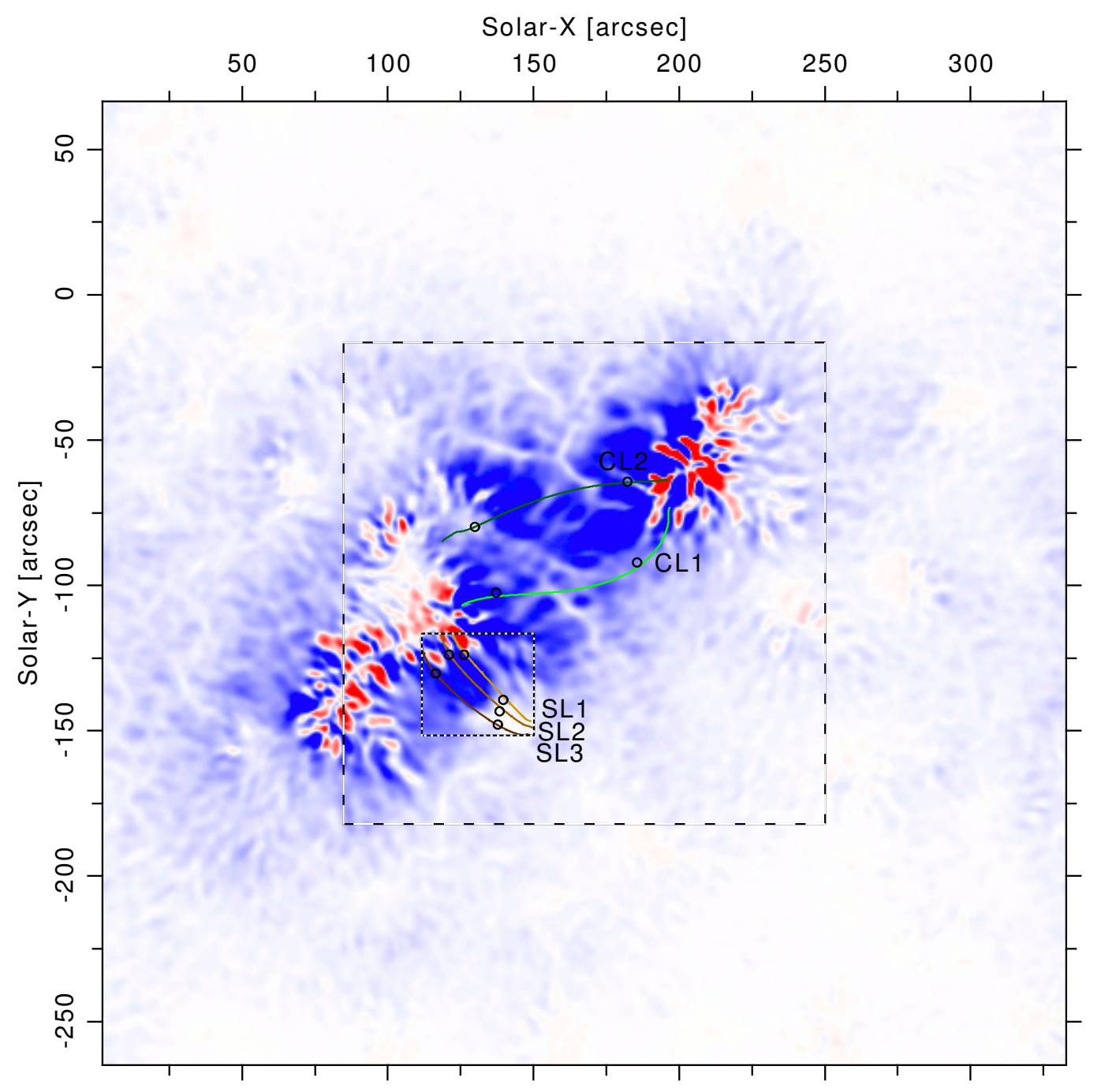

Figure 4.3: Vertical Poynting flux at a height of $3 \mathrm{Mm}$. The circles mark the footpoints of the hot AR core loops and the solid lines their respective field line. Upwards oriented vertical flux is colored in blue and downwards flux in red, both in linear scale with a saturation level of $\pm 50^{\prime} 000 \mathrm{~W} / \mathrm{m}^{2}$. The dashed square indicates the hot AR core area (c.f. Fig. 1.4 and 3.1), while the dotted rectangle outlines the FOV of only the short loops SL 1-3, see Sect. 4.2.1.3.

magnetic energy from the photosphere into the corona, where it can then be dissipated (c.f. Fig. 4.3).

The vertical component of the Poynting flux is highly structured at the photospheric level, as are the magnetic field and the horizontal velocities. With increasing height, the magnetic field is smoothed out by the magnetic expansion and the contrast in the Poynting flux also smoothens quickly. In order to become independent of the large fluctuations in the photosphere, and also to have a reliable and smooth 
estimate of the magnetic field lines, we choose the height where the temperature reaches a value of $75^{\prime} 000 \mathrm{~K}$ as point of reference for the footpoints of a field line. This temperature lies above the chromosphere but still within the transition region that is typically located at heights between $3 \mathrm{Mm}$ and $5 \mathrm{Mm}$ in our model.

A coronal loop typically is a roughly semi-circular structure with a field inclination of 0 to 30 degrees in the lower $3 \mathrm{Mm}$ of the atmosphere, that is below the transition region. Because the magnetic field can vary strongly there, a good estimate of the loop length $L$ would disregard a field line that expands to remote regions by following directional changes in the chromospheric magnetic field. Scaling laws are usually based on observations and only use the visible coronal part of a loop to determine its length. For this analysis, the loop length $L$ is defined as the distance between both loop footpoints plus the distance to the photosphere, which we compute by assuming an average inclination of 20 degrees for the magnetic field below the transition region. If we assume a different average inclination between 0 and 30 degrees below the transition region, the results of this analysis deviate only marginally, because the loop length is dominated by the coronal part.

\subsection{Statistical analyses of coronal field lines}

For a statistical analysis of coronal field lines we first need to select a sample of field lines that represent well the AR corona. From our model we extract not only the field lines of the hot coronal loops, but also field lines rooted all over the AR region core. The seed points for the field-line tracing are distributed along a vertical cut through the domain, located roughly in the middle between the two main polarities of the AR. Selected field lines are a) longer than $18 \mathrm{Mm}$ in total, b) shorter than $150 \mathrm{Mm}$, and c) reach at least to the given minimum temperature of $75^{\prime} 000 \mathrm{~K}$ somewhere below $18 \mathrm{Mm}$ height. If a field line doesn't fulfill one of these conditions it either closes below the corona, is located in a cool quiet region and wouldn't produce coronal emission in the spectral lines we are interested in, or it spans high up into the corona, where the magnetic perturbations from the bottom layer might not have sufficiently propagated to, yet. Also we prevent open field lines from being selected. In addition to this rather "global" ensemble, we added for each hot $A R$ core loop one field line that crosses the maximum intensity of that loop, as well as about 200 field lines neighboring these most prominent loops (c.f. Fig. 4.3). The 67'000 field lines we use for this analysis have apex heights of about 6 to $80 \mathrm{Mm}$, while the most prominent loops reach heights between 10 and $22 \mathrm{Mm}$. 


\subsubsection{Coronal energy conversion}

In Sect. 4.2.1 we analyzed the coronal energy conversion process only with spatial averages over different subvolumes. In this section we now turn to the coronal energy conversion process, in particular the Ohmic heating and its relation to the photospheric driving and to coronal temperatures, by looking at statistical ensembles of coronal field lines.

\subsubsection{Poynting flux as energy source}

Now we investigate the influence of the Poynting flux on the coronal energy input. The Poynting flux into a field line is computed as the sum of the directed flux at both field-line footpoints, while an upward flux is positive and a downward flux is negative. This sum gives a measure for the net vertical Poynting flux that actually remains in the corona, if that value is positive (58'000 field lines, c.f. Fig. 4.4). About one seventh of the selected field lines also show a negative net flux (9'000 field lines), which stands for a magnetic energy outflow from the corona downwards. Nonetheless, the horizontal average of the divergence of the Poynting flux is positive in all heights within the hot AR core loops FOV, c.f. Fig. 4.1. In Fig. 4.4 we relate the vertical net Poynting flux into a field line to the volumetric Ohmic heating integrated between both field-line footpoints, not taking into account the part below the transition region. The main bulk of the population is of positive net Poynting flux (dark blue). Still, also negative net Poynting flux occurs (red), but the strongest Poynting fluxes are all positive, as can be seen on the right end of the distribution in Fig. 4.4.

A negative net Poynting flux (red points in Fig. 4.4) can be explained by e.g. coronal induction or Lorentz work that increases the magnetic energy locally, which then can be transported downwards. Also a likely process is that the negative flux (produced at the lower boundary) cancels out with positive flux from neighboring field lines due to the magnetic expansion in the corona. In total, the mean Poynting flux for the full FOV of our model is nonetheless positive at every height. All hot AR core loops (marked by symbols) lie within the positive net flux population on the upper right, except for CL 2. All of them have a positive net Poynting flux, except for SL 3 that is untypical The loop SL 3 is relatively cool and doesn't show much emission in EUV and X-Rays and is therefore not a typical hot coronal AR loop, see also Chap. 3. The same is true for CL 2 that is not a developed hot loop and that lies outside the main coronal emission structures. CL 1 lies farther away from the equality line (gray line in Fig. 4.4), which indicates that this loop uses a smaller fraction of the incoming Poynting flux for Ohmic heating than the other hot AR core loops. Due to the particularly high incoming 


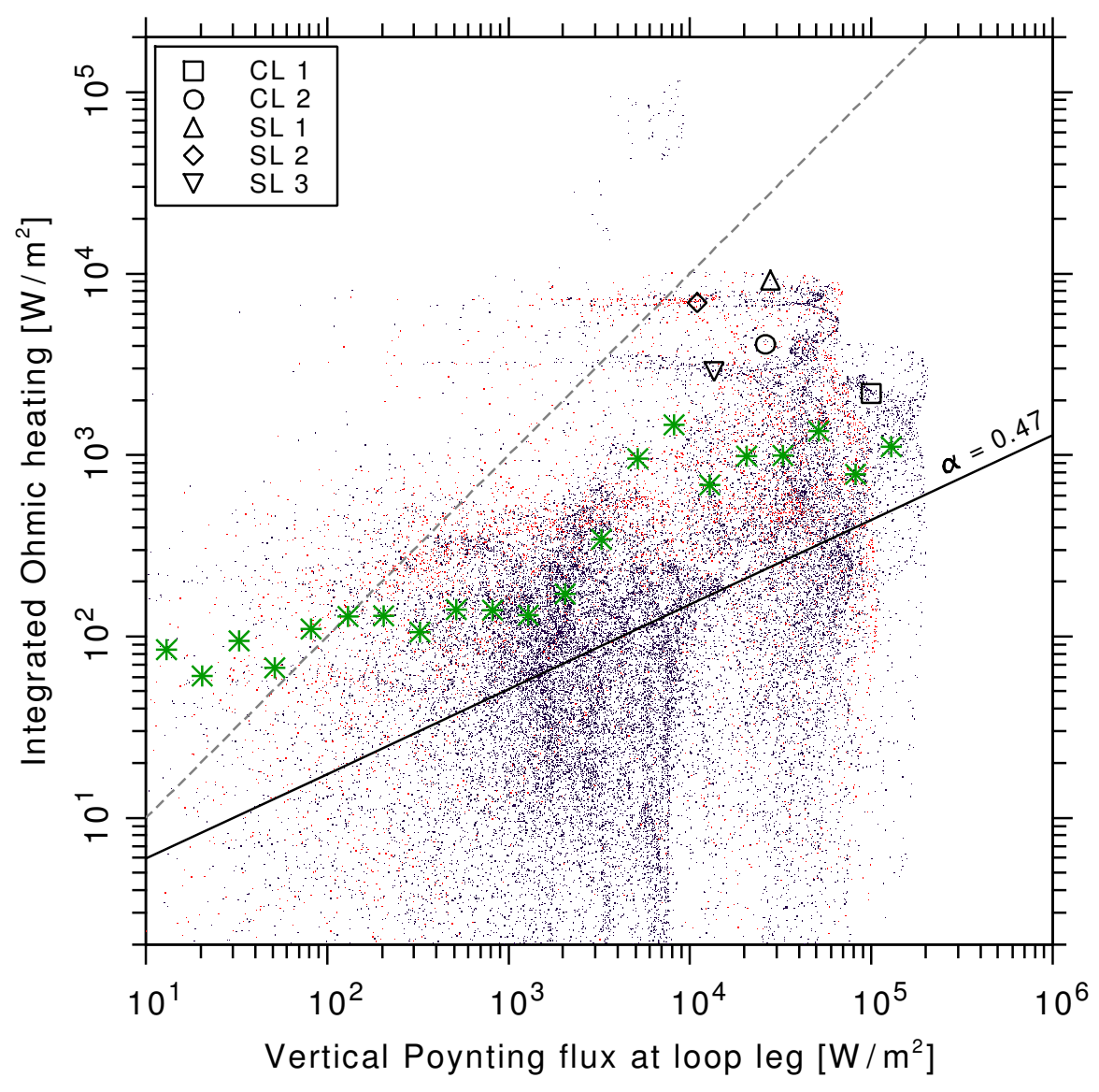

Figure 4.4: Vertical net Poynting flux into a field line versus the volumetric Ohmic heating integrated along that field line. The gray dashed line indicates the equality, where all of the incoming Poynting flux would be converted to Ohmic heat. We apply a least-absolute-deviation (LAD) fit to the positive net flux data points (black line). The negative net flux data points (red) are overplotted over the positive ones (dark blue). The green stars indicate the mean Ohmic heating within the corresponding bins in Poynting flux. See Sect. 4.3.1.1.

Poynting flux, CL 1 still can reach Ohmic heating rates as high as the other loops.

We fit a scaling law with an exponent of $\alpha=0.47 \pm 0.06$ to the positive data points (c.f. Fig. 4.4). The error is computed from the standard deviation of the LAD fit and by estimating the width of the data distribution to four orders of magnitude in the horizontal direction. This is justified by the relatively low scatter of the mean values for each Poynting flux bin (green stars). The positive slope of the fit is consistent with a relation between the volumetric Ohmic heating integrated along a coronal field line and the vertical Poynting flux $\mathcal{P}_{z}$ at the 
field-line footpoints of the form

$$
F_{\text {ohm }} \sim \mathcal{P}_{z}^{\alpha}
$$

where $\overrightarrow{\mathcal{P}}=\eta(\vec{\nabla} \times \vec{B}) \times \vec{B}+(\vec{u} \times \vec{B}) \times \vec{B} / \mu$ with the velocity $\vec{u}$, the magnetic field vector $\vec{B}$, the magnetic diffusivity $\eta$, and the permeability $\mu$. Above a height of $2 \mathrm{Mm}$, where all the footpoints of our field lines are located, the Poynting flux is largely dominated by the second term that reflects transversal perturbations in the magnetic field. As the main distribution of data points is clearly located below the equality line, we conclude that usually not all of the Poynting flux in our model is converted to Ohmic heat. The hot AR core loops SL 1-3 and CL 2 convert a larger part of their incoming Poynting flux into Ohmic heat as compared to the average field lines.

Some of the remaining upwards magnetic energy transport that is not dissipated could e.g. increase the magnetic field stress in the corona and let it evolve away from a potential field configuration, as well as it can lead to an expansion of the magnetic structures into the upper corona. For instance, we found that the large AR core loops CL 1 and 2 are still rising with about $2 \mathrm{~km} / \mathrm{s}$ at the loop top (Bourdin et al. 2013). Also there is a net upwards mass transport in the middle of the $A R$ core between the two main polarities.

We perform now the same analysis with the Ohmic heating per particle instead of the volumetric one, see Fig. 4.5. In contrast to Fig. 4.4 we find here, that the small population of negative net vertical Poynting flux (red) separates from the main bulk of the positive net flux (dark blue) population towards smaller heating values. Again, we overplot a LAD fit to a scaling law that gives an exponent of $0.44 \pm 0.07$ (black solid line). This value is consistent within its error with the exponent found from the volumetric Ohmic heating analysis.

As in Fig. 4.4 the hot AR core loops (marked by symbols) are found at the upper right end of the distribution. In both Fig. 4.4 and 4.5 the hot loops clearly have a stronger heating than the scaling law fitted through all data points would predict. All in all this is good, because a positive exponent $\alpha$ supports a possible correlation between the net Poynting flux into a field line and its temperature, via the Ohmic heating in the corona, which we would expect.

\subsubsection{Ohmic heating integrated along field lines}

For the same ensemble of field lines as in Sect. 4.3.1.1 we investigate in Fig. 4.6 now the Ohmic heating per particle. We find here that the population of hot field lines mainly follows a scaling law of the form

$$
T_{\max } \sim F_{\text {Ohm }}^{2 / 7},
$$




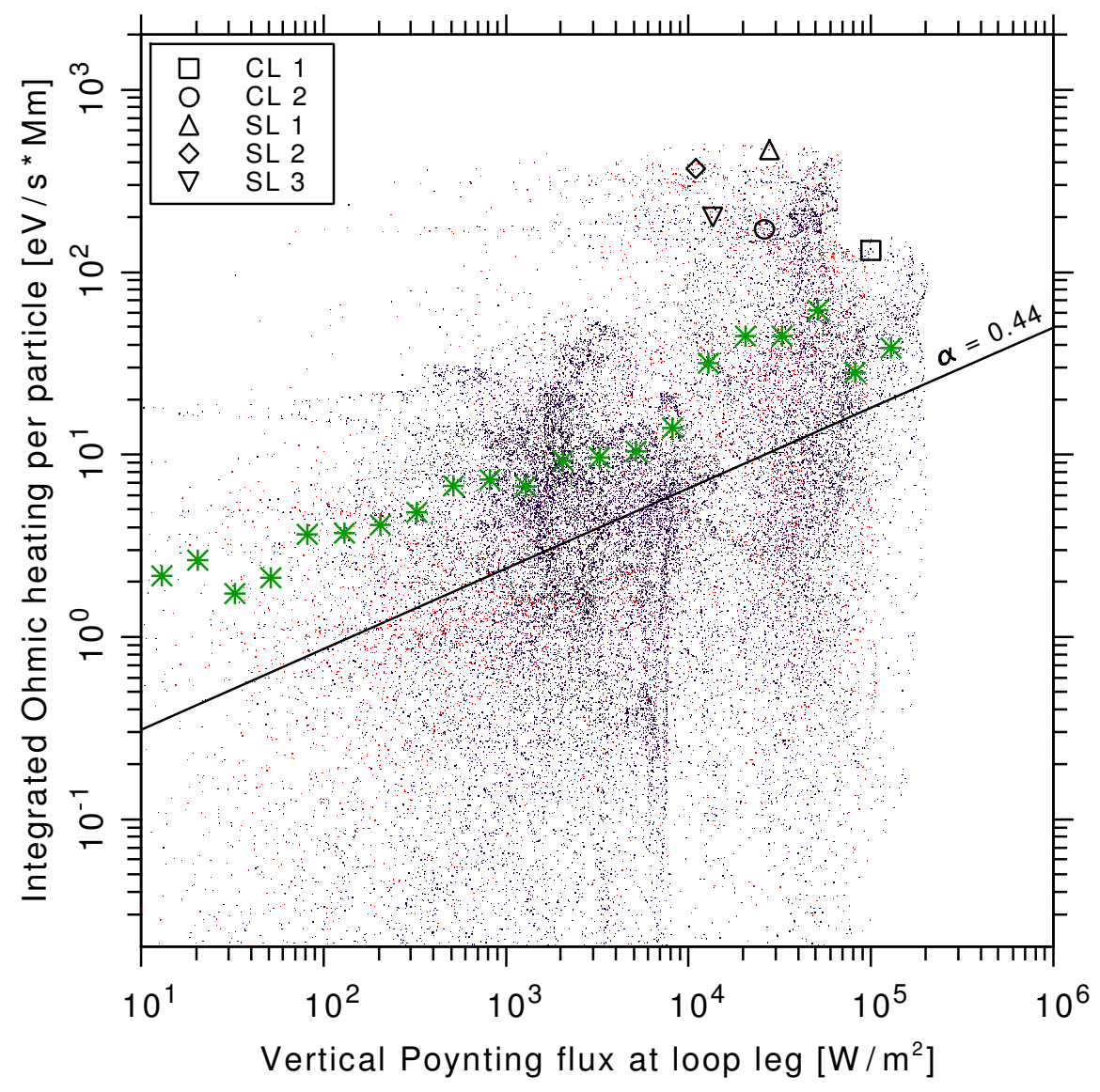

Figure 4.5: Same as Fig. 4.4 except that here the Ohmic heating per particle integrated along a field line is used.

where the maximum temperature $T_{\max }$ relates to the Ohmic heating per particle integrated along that field line $F_{O h m}=\int H_{O h m}(s) \cdot d s$. This fits to the RTV scaling laws, Eqns. 4.3.8 and 4.3.11 that will be discussed in Sect. 4.3.2.

While the distribution of data points in Fig. 4.6 is relatively narrow for the high heating rates and high temperatures, the distribution becomes much broader for field lines with a maximum temperature below 0.5 MK. These cooler field lines have insufficient heating to become hot and are not quasi-static. Therefore, these will not follow the RTV scaling law and one expects scatter.

In Fig. 4.7 we do the same analysis as in Fig. 4.6 but using the volumetric Ohmic heating rate integrated along each field line. In contrast to Fig. 4.6 the width of the distribution is significantly broader here for all field lines with a maximum temperature below $1 \mathrm{MK}$. Still, the population seems to follow the slope of $2 / 7$, while the population has the tendency to extend towards higher heating rates. Obviously, the Ohmic heating per particle is better comparable with a scaling 


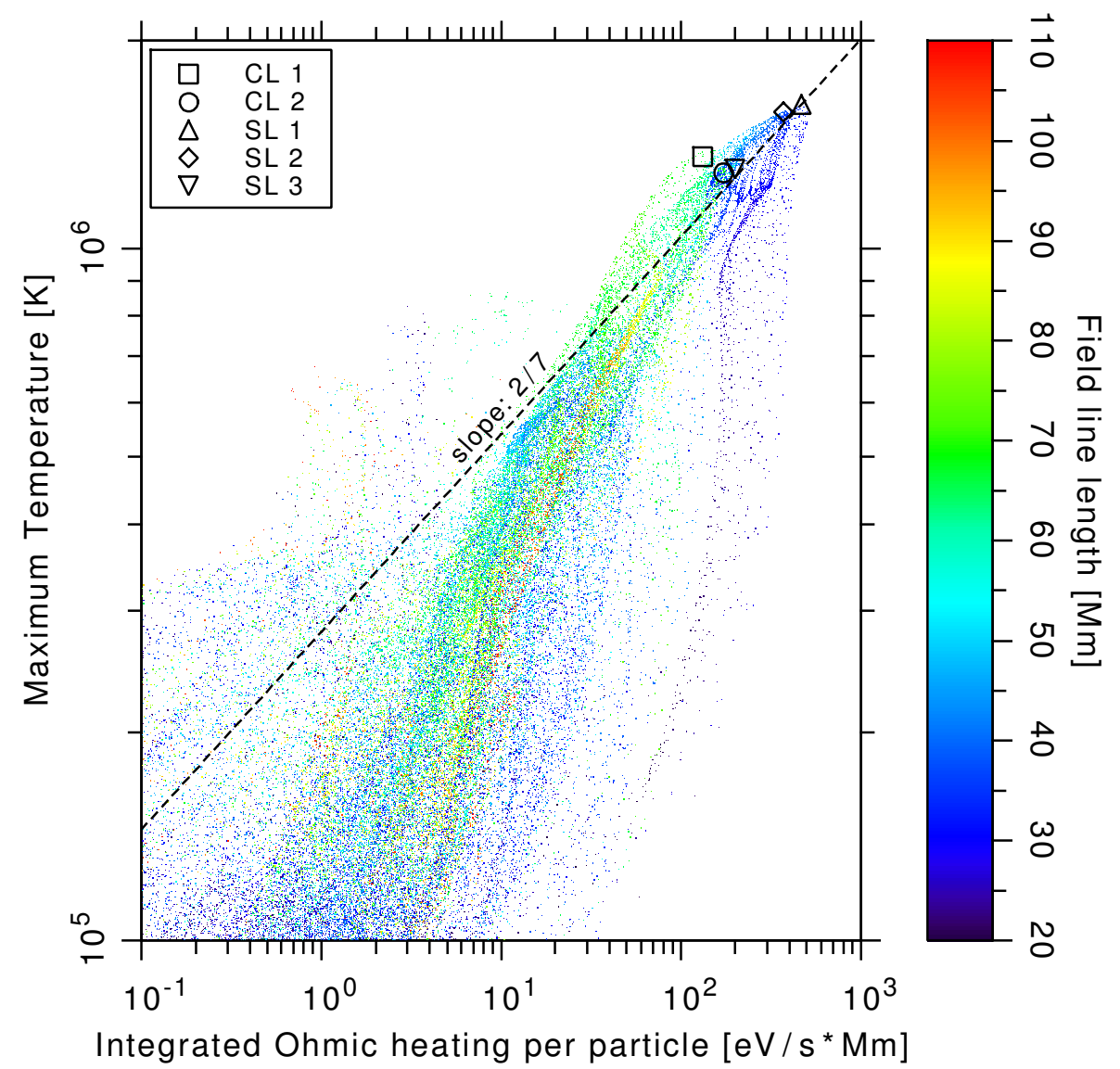

Figure 4.6: Ohmic heating per particle integrated along a field line versus its maximum Temperature. The hottest loops (marked with symbols) all have a high Ohmic heating per particle. The three loops with the strongest EUV emission (SL $1+2$ and $C L 1$ ) have the highest temperature as compared to their neighboring data points. The dashed black line indicates the exponent of 2/7 with the parameters of the loop SL 1 as anchor point, see Sect. 4.3.1.2.

law in the sense that the distribution is narrower and that the scaling is more uniform regarding field-line lengths, see for example the yellow and red population just below the symbols (85-110 Mm length), which has a non-constant slope in the volumetric Ohmic heating case.

\subsubsection{Mean Ohmic heating}

In a last analysis regarding this subject, we investigate the relation between average quantities along a field line, instead of maximum and integrated quantities. The relation in Fig. 4.8 between the mean Ohmic heating per particle and the mean temperature along a field line is visible, even though it is less clear than in Fig. 4.6 


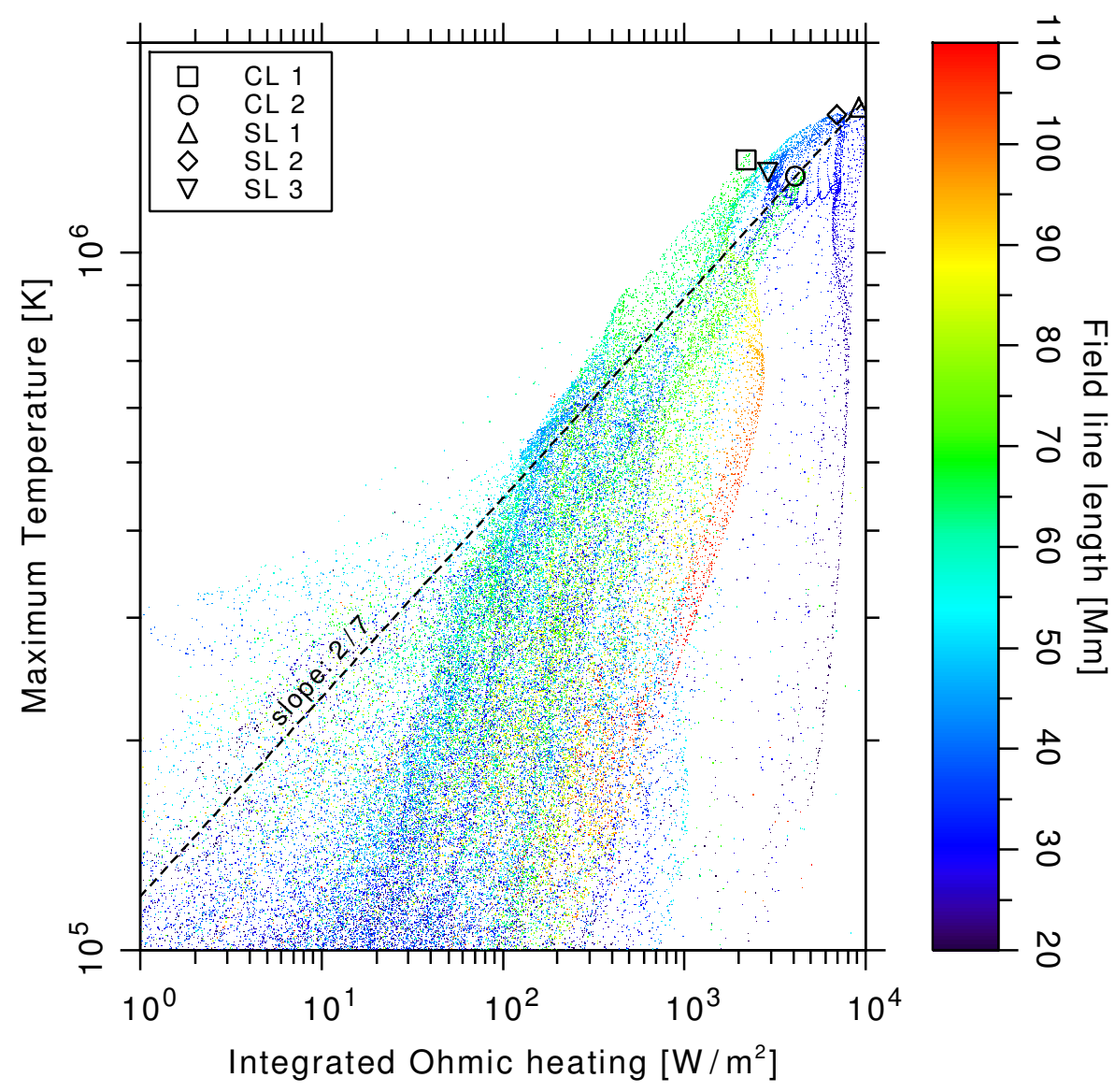

Figure 4.7: Same as Fig. 4.6 but showing the volumetric Ohmic heating integrated along a field line versus its maximum Temperature. The highest-temperature loops (SL $1+2$ and $\mathrm{CL} 1$ ) lie exactly at the local maxima of their surrounding population.

and 4.7 .

Nonetheless, we see here a clearer relation between the field-line length (color coded in Fig. 4.8) and the mean heating, as compared to Fig. 4.7. The short and hot loops SL $1+2$ show the strongest mean heating, while the longer loops $\mathrm{CL} 1+2$ have a 10 times lower average heating, even though their lengths (35 and $70 \mathrm{Mm}$ ) differ only by a factor of 2 . Still, they have roughly the same average temperature. We also find the same results, when we use the volumetric heating instead of the heating per particle.

\subsubsection{Relation to magnetic field strength}

Also of high interest is the connection between the field-line length (and hence the field line's tendency towards high or low coronal temperature) in relation to the 


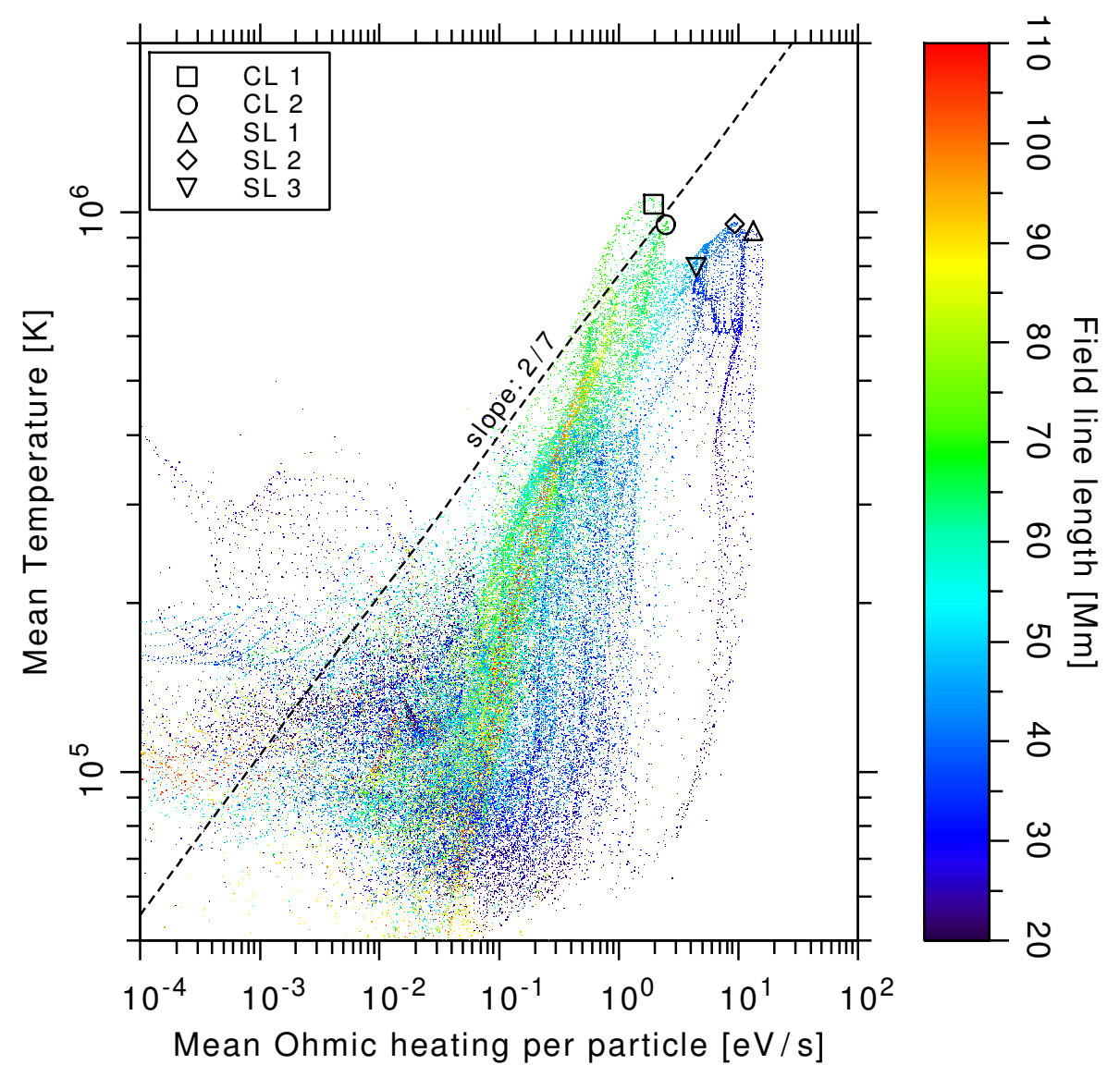

Figure 4.8: Mean Ohmic heating per particle integrated along a field line versus its mean Temperature. The mean is computed here as the heating integrated along a field line (as is Fig. 4.6) divided by the integration path length. The field lines with a heating rate above $10^{-1} \mathrm{eV} / \mathrm{s}$ are roughly ordered according to their length. See Sect. 4.3.1.3.

magnetic flux density at the footpoints of the field line. As mentioned in Sect. 2.3.4 there are strong fluctuations of the vertical magnetic flux density in the photospheric layer. For this analysis it is important to be independent of these strong fluctuations that would anyway not reach into the corona. Therefore we choose $300 \mathrm{~km}$ as the reference height for the magnetic flux into a field line, which is roughly one photospheric pressure scale-height above the photosphere, but still below the chromosphere in our model.

In Fig. 4.9 we display the mean of the vertical magnetic flux density values at both field-line footpoints versus the volumetric Ohmic heating integrated along each field line. The field-line length (color coded) is taken between the reference heights where the temperature reaches $75^{\prime} 000 \mathrm{~K}$, plus the extension towards the 


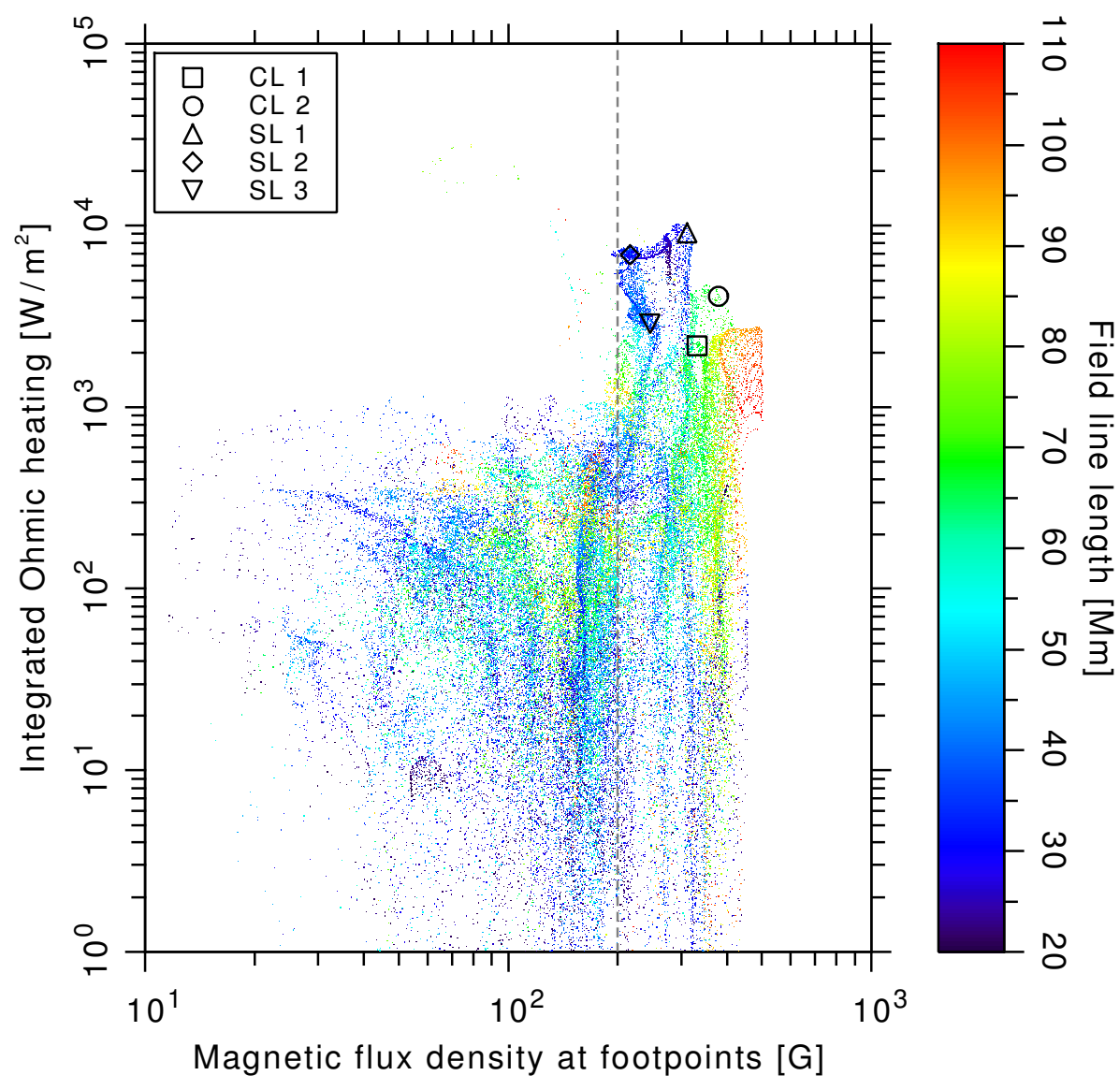

Figure 4.9: Magnetic flux density at the footpoints located at $z=300 \mathrm{~km}$ height versus volumetric Ohmic heating integrated along the field line. The hot loops $\mathrm{SL} 1+2$ lie within the high heating rates population. The vertical gray dashed line indicates the magnetic flux density threshold of $200 \mathrm{G}$, see Sect. 4.3.1.4.

photosphere.

Below a critical magnetic flux density of roughly $200 \mathrm{G}$ (gray dashed line in Fig. 4.9), we find a wide distribution of flux densities with no general correlation to the integrated Ohmic heating nor the field-line length. Nevertheless, above this critical flux density almost all strongly heated field lines are located. We do not see a strong correlation between the footpoint flux density and the coronal heating rate, but the field-line lengths clearly separate spanning from the long field lines $(100 \mathrm{Mm}$, red at the lower right) with relatively low Ohmic heating to the short field lines $(30 \mathrm{Mm}$, dark blue at the upper left) with the strongest Ohmic heating. Altogether, this supports the interpretation that the magnetic energy input into a field line is responsible for the coronal Ohmic heating and that a threshold of about $200 \mathrm{G}$ in the footpoint flux density is a prerequisite to provide a sufficient net 
vertical Poynting flux. Also a sufficiently strong flux density allows for a field line that reaches into the corona, instead of closing at low height to one of the many neighboring opposite polarities of less than $200 \mathrm{G}$.

\subsubsection{Scaling laws for coronal loop properties}

With the previous findings, we have good indications for relations of the coronal Ohmic heating to the field-line length, the footpoint magnetic flux density, and hence also the vertical net Poynting flux. We now want to compare our model field lines with theoretical scaling laws relating plasma parameters (like the density, the temperature, or the pressure) of the field line to the energy input and field-line length. Such scaling laws are partly also derived from observations (Rosner et al. 1978; Serio et al. 1981). Rosner etal. (1978) were the first to derive the RTV scaling laws (named after the authors)

$$
\begin{aligned}
& T=c_{T} \cdot(p L)^{1 / 3} \cdot E_{T}, \\
& H=c_{H} \cdot p^{7 / 6} L^{-5 / 6} \cdot E_{H},
\end{aligned}
$$

for the temperature $T$ and the heating rate $H$ along a hot coronal loop. Here, the heating rate $H(s)$ is assumed to be constant along a loop and is irrespective of the actual mechanism that delivers the energy. For the standard RTV scaling law with spatially constant $H$, the correction factors $E_{T}$ and $E_{H}$ are both equal to 1 . The constant factors in SI units are:

$$
\begin{aligned}
c_{T} & =1400 \mathrm{~K}\left(\mathrm{~s}^{2} / \mathrm{kg}\right)^{1 / 3}, \\
c_{H} & =9.8 \cdot 10^{3} \mathrm{~J} / \mathrm{m}^{3} .
\end{aligned}
$$

The RTV equations can be rewritten as

$$
\begin{aligned}
p & =c_{H}^{-6 / 7} \cdot H^{6 / 7} L^{5 / 7} \cdot E_{H}{ }^{-6 / 7}, \\
T & =c_{T} \cdot c_{H}^{-2 / 7} \cdot H^{2 / 7} L^{4 / 7} \cdot E_{T} E_{H}{ }^{-2 / 7}
\end{aligned}
$$

With the ideal gas law $p=2 n_{e} k_{B} T$ we find

$$
n_{e}=\frac{1}{2 k_{B}} c_{H}^{-4 / 7} c_{T}^{-1} \cdot H^{4 / 7} L^{1 / 7} \cdot E_{T}^{-1} E_{H}^{-4 / 7}
$$

for the electron number density $n_{e}$.

In our model we have a variable Ohmic heating $H(s)$ deposited along the field lines, so it makes more sense to use the integrated heating

$$
F_{H}=\int H(s) \cdot d s
$$


For a roughly constant $H$, this simplifies to $F=H \cdot L$. Under this assumption Eqns. 4.3 .8 and 4.3 .9 become:

$$
\begin{aligned}
T & =c_{T} \cdot c_{H}^{-2 / 7} \cdot F_{H}{ }^{2 / 7} L^{2 / 7} \cdot E_{T} E_{H}{ }^{-2 / 7} \\
n_{e} & =\frac{1}{2 k_{B}} c_{H}^{-4 / 7} c_{T}^{-1} \cdot F_{H}{ }^{4 / 7} L^{-3 / 7} \cdot E_{T}{ }^{-1} E_{H}{ }^{-4 / 7} .
\end{aligned}
$$

Eqn. 4.3 .8 can be rewritten as a realtion of the loop heating

$$
H=c_{H} \cdot c_{T}^{-7 / 2} \cdot T^{7 / 2} L^{-2} \cdot E_{T}^{-7 / 2} E_{H} .
$$

\subsubsection{Scaling laws in 3D model}

\subsubsection{Heating and loop length}

First, we plot in Fig. 4.10 the field-line length versus the average of the volumetric Ohmic heating, both quantities represent only the coronal part, as defined in the Sects. 4.2.1.3 and 4.3.1.4. We find a broad distribution, where field lines with footpoint flux densities of 300-400 G appear practically everywhere, but there is a concentration in the upper center around $75-100 \mathrm{Mm}$ field line length that shows significantly high heating rates. As we go to shorter field lines, we see that the footpoint flux density decreases to values around $200 \mathrm{G}$ for field-line lengths of $20 \mathrm{Mm}$. At the same time the Ohmic heating rises, following the trend of the overplotted RTV scaling law (gray dashed line) that we get from sample parameters of a hot coronal loop with a maximum temperature of $T_{\max }=1.6 \mathrm{MK}$. The three hottest coronal loops (SL $1+2$ and CL 1) follow well this RTV scaling law. The same relation holds also between both warm loops (SL 3 and CL 2). As we already demonstrated in Fig. 4.9 field lines with footpoint flux densities below a critical limit of $200 \mathrm{G}$ do not show particularly high Ohmic heating. Nonetheless, they can span over short and long distances into the corona. For field lines longer than $150 \mathrm{Mm}$ with flux densities of about $150 \mathrm{G}$ (turquois), we still find some that reach towards the scaled properties of much shorter warm coronal field lines (c.f. Fig. 4.10). If that scenario is persistent, more warm loops of lengths above $150 \mathrm{Mm}$ will form in our model that will span high up into the corona. Besides that, there is also a large population of field lines with low footpoint flux densities that are simply not sufficiently heated and that are currently cooling down. This is consistent with coronal observations, where only a minority of coronal field lines is actually seen as loops that are bright in EUV or X-ray emission lines, and a majority of field lines is not. 


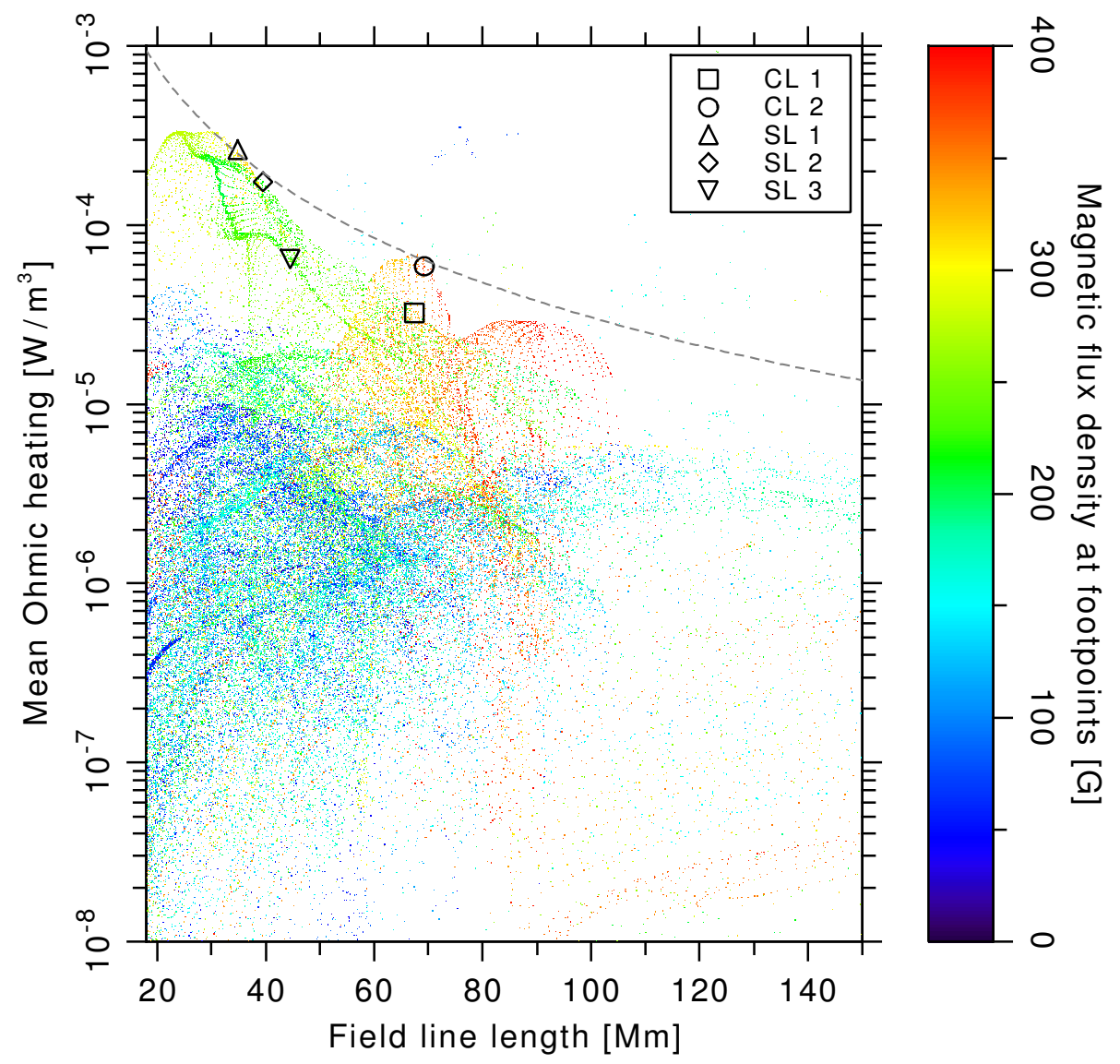

Figure 4.10: Field-line length versus volumetric Ohmic heating averaged along the field line, together with the magnetic flux density at the field-line footpoints (color coded) at a height of $300 \mathrm{~km}$. The gray dashed curve represents the RTV scaling law following Eqn. 4.3 .13 for a mean Ohmic heating $\langle H\rangle=F_{H} / L$ of a sample coronal loop, see Sect. 4.3.3.1.

\subsubsection{Loop temperature}

The RTV scaling law itself can also be tested against the model directly. We do this by comparing the maximum temperatures of the coronal loop, once taken directly from the model, and once calculated from the model properties, i.e. the energy input into a structure and its field-line length, using the RTV scaling law Eqn. 4.3.11. Because RTV assumes a constant heating rate, we use here the average heating rate $\langle H\rangle=F_{H} / L$ for comparison. In Fig. 4.11 we present the distribution of the model values in relation to the RTV scaling law, where the black solid line indicates the equality of both quantities.

In hydrostatic equilibrium and with a prescribed heating, the loop-top temperature $T_{\max }$ can be derived for a ID loop model in thermal equilibrium and with a static 


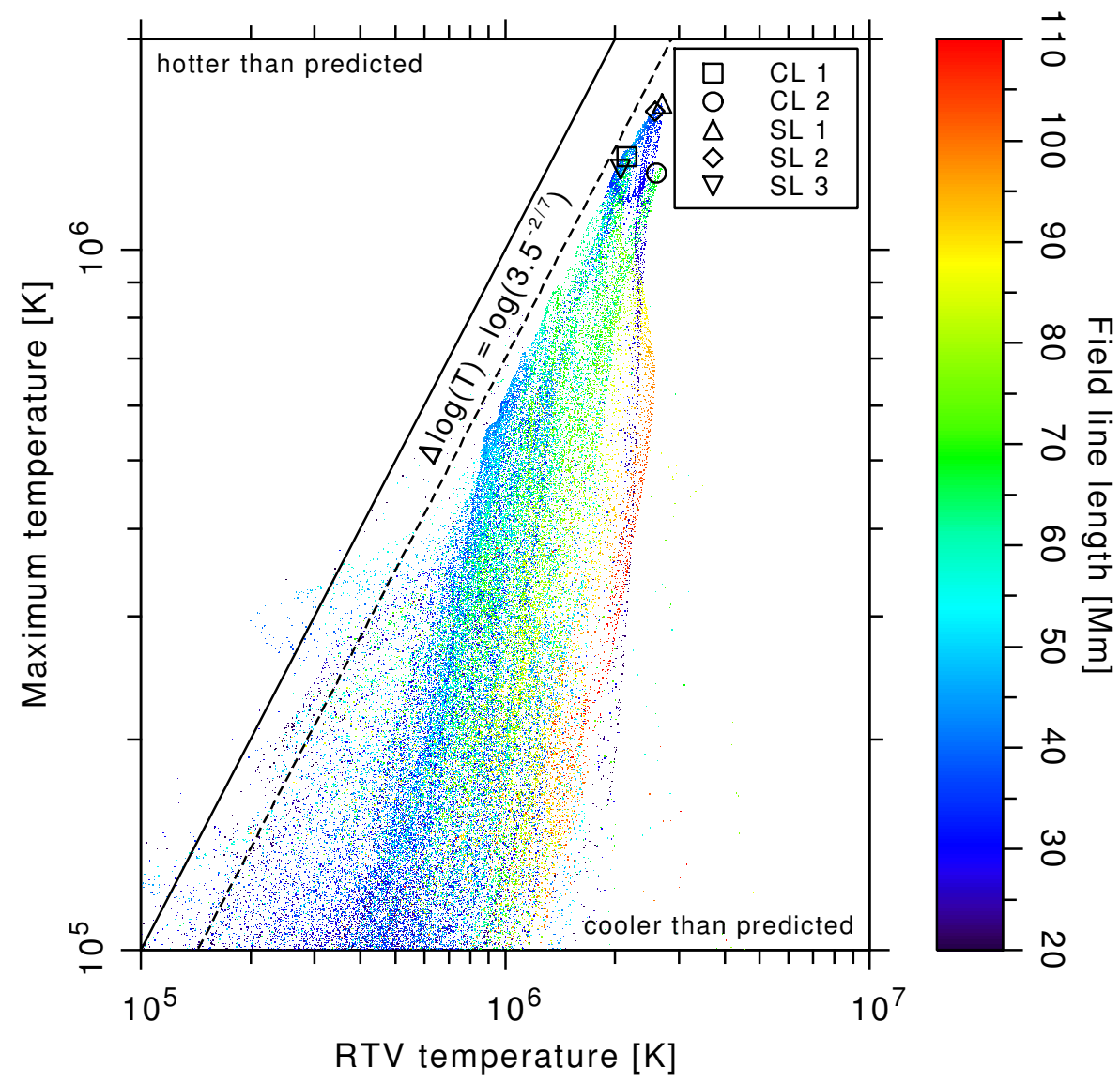

Figure 4.11: RTV temperature versus maximum temperature along a field line. The solid line indicates the equality to the RTV scaling law, while the dashed line indicates a correction factor of $E_{H}=3.5$, see Sect. 4.3.3.2.

energy balance including radiative losses and heat conduction. As a result, the RTV heating (Eqn. 4.3.4) is derived with a constant correction factor $E_{H}=3.5$ (black dashed line, Priest 1982). This correction factor corresponds to a constant shift in logarithmic coordinates (dashed line). The slope of both lines reflects an exponent of $2 / 7$.

We find a good match in the scaling for the hot end of the distribution, starting with temperatures of $0.5 \mathrm{MK}$ and up to our hottest model loops (symbols) at around 1.7 MK, see Fig. 4.11. Also here, a large population of field lines is cooler than predicted, which is not a contradiction, because these cool field lines are not covered by the RTV scaling laws that were derived only from hot loops. We also find that the temperature relation Eqn. 4.3.11 including a constant correction factor $E_{H}$ significantly fits better to the strongly heated coronal field lines we have in our model (c.f. Fig. 4.11). 


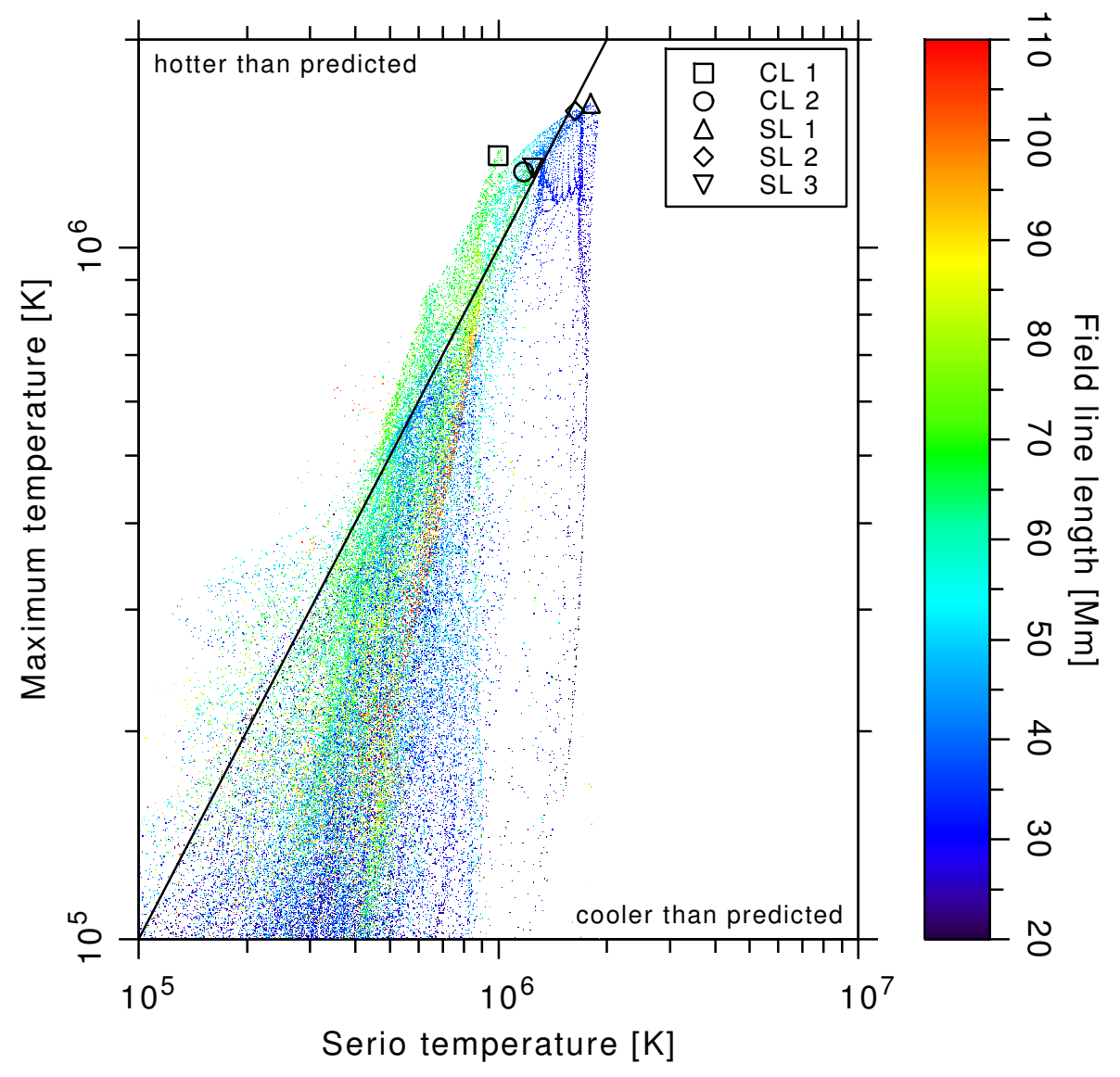

Figure 4.12: Serio-RTV temperature versus maximum loop temperature.

For the case of a non-constant heat input, in particular for an exponential decay with height, Serio etal. (1981) derived a modification for the RTV scaling laws. The Eqns. 4.3 .3 to 4.3 .12 account for different pressure and heating scale heights $\left(s_{P}\right.$ and $s_{H}$ ) by the correction factors

$$
\begin{aligned}
E_{T} & =\exp \left(-0.04 \cdot L\left(2 / s_{H}+1 / s_{P}\right)\right), \\
E_{H} & =\exp \left(0.5 \cdot L\left(1 / s_{H}-1 / s_{P}\right)\right)
\end{aligned}
$$

that we inspect further in the appendix A

In Fig. 4.12 we plot this corrected theoretical prediction from the model field-line parameters versus the maximum temperature of that same field line. The hot end of the distribution of temperatures fits well to the purported scaling law. The distribution becomes significantly narrower with the non-constant Serio et al. (1981) correction factors $E_{T}$ and $E_{H}$. Furthermore, these factors also compensate the constant offset of the RTV scaling law in Fig. 4.11. Therefore, as to be expected, the Serio scaling law fits better to the overall temperature distribution of our model 


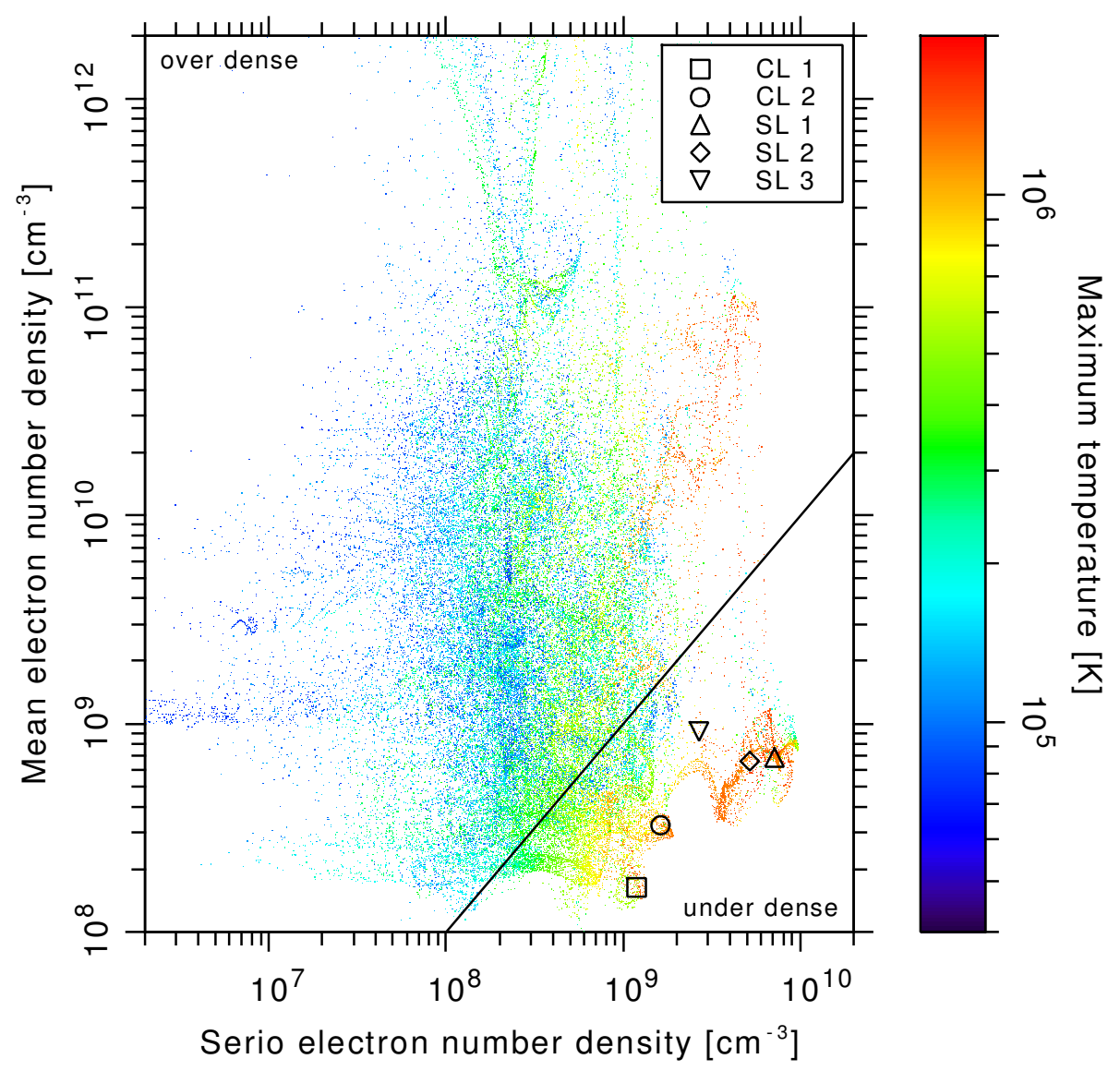

Figure 4.13: Electron number density from the Serio-RTV scaling law versus the mean density along the coronal part of a loop. The black solid line indicates the equality of both quantities. See Sect. 4.3.3.3.

field lines. On the other hand, the Serio scaling law shows an S-shaped deviation from the double-logarithmic equality line, which indicates a systematic error that is not present in the original RTV scaling law.

\subsubsection{Loop density}

The average electron number density of our model field lines is computed as $\left.<n_{e}\right\rangle=L^{-1} \cdot \int n_{e}(s) \cdot d s$. In Fig. 4.13 we show this average number density versus the Serio et al. (1981) scaling law number density $n_{e}$ that is computed from the integrated heating along a field line $F_{H}$, the correction factors $E_{T}$ and $E_{H}$ (Eqns. 4.3 .14 and 4.3.15), and the loop length $L$, see Eqn. 4.3.12. The data points below the equality line (black) are field lines that have a lower density than the scaling law would predict ("under dense"), while the points above are denser than predicted ("over dense"). 
We find here that the hottest AR core loops (SL $1+2$ and CL 1) are most under dense, while the warm loops (SL 3 and CL 2) are less under dense. Still, most of our model field lines are over dense, while they are also cooler than predicted, c.f. Fig. 4.12. This is consistent with earlier works, where the tendency of cooler loops ( $1 \mathrm{MK})$ to be over dense was found (Aschwanden et al. 1999), while hotter loops ( $2 \mathrm{MK}$ ) were observed with Үонкон and found to be under dense (Porter and Klimchuk 1995). For our model loops we find the same tendency, while there is still a minority of data points that do not fulfill this general trend. We find that our model densities fit best to the values predicted by Serio et al. (1981) around a maximum temperature of roughly $0.8 \mathrm{MK}$, which is hence the over/under dense turnover temperature for our model field lines.

From our MHD model data we know that the average density is lower inside the hot $A R$ core subvolume than above, which is reflected by the fact that the forming hot loop CL 1 has an average density lower than most other field lines. The reason is that the hot $A R$ core subvolume lost some mass towards the chromosphere (c.f. Fig. 4.1) and was not fed with mass from above, because the magnetic field topology shields this subvolume from incoming plasma downflows. Altogether, this is perfectly consistent, because if the heating is assumed to be roughly uniform in a coronal loop (in time average), denser loops receive a lower heating per particle, which should actually result in lower temperatures for denser loops as compared to less dense or under dense loops.

\subsubsection{Parameterization of Ohmic heating}

\subsubsection{1 van Ballegooijen scaling law}

We want to investigate further possible scaling laws for the Ohmic heating rate. For instance, van Ballegooijen et al. (2011) have conducted box model simulations of individual coronal loop structures (flux tubes) using a stratified atmosphere. In these models, driving motions are applied on the two opposite ends of the cuboid box, which encompasses the non-curved straight loop. Alfvén waves and MHD turbulences heat the coronal part of the loop. From a parameter study, the following relation to the loop length, the magnetic flux density at the photospheric boundary, and the driving motions is inferred:

$$
\begin{aligned}
Q_{c o r}= & 2.9 \cdot 10^{-3} \cdot\left(0.45+\frac{33 \mathrm{~s}}{\tau_{0}}\right) \cdot\left(\frac{B_{c o r}}{50 \mathrm{G}}\right)^{\beta=0.55} . \\
& \cdot\left(\frac{L}{50 \mathrm{Mm}}\right)^{\gamma=-0.92} \cdot\left(\frac{v_{R M S}}{1.48 \mathrm{~km} / \mathrm{s}}\right)^{\delta}\left[\mathrm{W} / \mathrm{m}^{3}\right]
\end{aligned}
$$




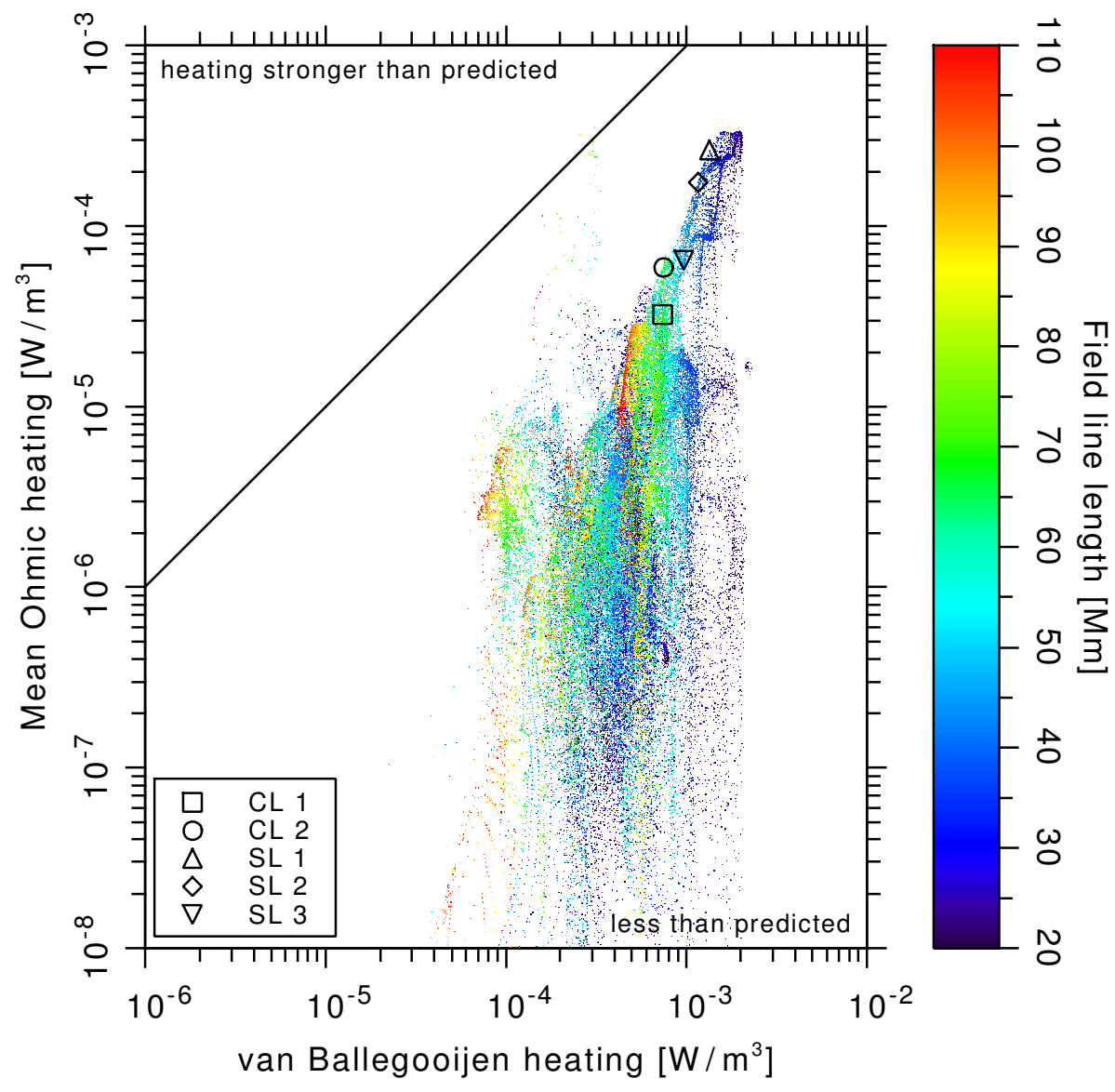

Figure 4.14: Mean Ohmic heating as predicted from the van Ballegooijen et al. (2011) scaling law versus the mean Ohmic heating along the model field lines. The black line indicates the equality between the predicted and the model values. See Sect. 4.3.4.1.

where $\tau_{0}=60-200 \mathrm{~s}$ is the correlation time of the driving motions. The average (root-mean-squared) velocity of the driving motions in our model is $v_{R M S}=1.242 \mathrm{~km} / \mathrm{s}$. For better comparability with Sects. 4.3 .4 .2 and 4.3 .4 .3 we use here a different value of $\delta=2.25$ instead of 1.65 for the exponent of the velocity relation. This change only results in a constant shift of all prediction values towards higher coronal heating and hence closer to the equality with our model data.

In Fig. 4.14 we compare the parameters of our model field lines with the prediction based on the van Ballegooijen et al. (2011) scaling law for the coronal heating. Basically, we find that practically all coronal field lines have a significantly lower heating rate than predicted. The short and hot AR core loops $(S L 1+2)$ have the highest prediction for the coronal heating, while the longer loops $(C L 1+2)$ are 
heated about ten times less. These strongest heated field lines form a population (from CL 1 to SL 1 , above $3 \cdot 10^{-5} \mathrm{~W} / \mathrm{m}^{3}$ ) that indicates a slope roughly twice as steep as the van Ballegooijen et al. (2011) scaling law would predict.

The length relation in Eqn. 4.3.16 seems to be partly responsible for the distribution of the data points within this most heated population. As one would expect from the inverse dependency on $L$, the shorter field lines (blue) have a higher predicted coronal heating than the longer field lines (turquois). This trend even continues to the longest coronal field lines (yellow and red) that are located left below the long hot coronal loop CL 1 .

For a coronal Ohmic heating below $10^{-5} \mathrm{~W} / \mathrm{m}^{3}$ we find a strong variation of the field-line parameters that doesn't allow conclusions on the slope of possible scaling laws. A dependency on the field-line length can still be seen, because the long (red) and the short (blue) field lines are roughly ordered from the left to the right. Overall, the match of the prediction to our model parameters is not good. The differences in comparison to our model can be explained by a different Alfvén travel time into the corona, different dissipation scales, the curvature of magnetic field in our model setup, and maybe also a different dissipation mechanism that is more comparable to slow magnetic diffusion instead of Alfvén waves or MHD turbulences.

\subsubsection{Rappazzo scaling law}

Based on MHD turbulence models that resemble the field-line braiding mechanism, Rappazzo et al. (2008) have found a different dependency of the Ohmic heating on the magnetic flux density and the loop length than van Ballegooijen et al. (2011). These different exponents $\beta=1.75, \gamma=-1.75$, and $\delta=1.25$, as well as the additional dependency $\omega=0.125$ on the mean field-line particle number density $n_{\rho}$, we test with a modified scaling law:

$$
\begin{aligned}
Q_{\text {turb }}= & 2.9 \cdot 10^{-3} \cdot \frac{120 \mathrm{~s}}{\tau_{A}+60 \mathrm{~s}} \cdot\left(\frac{n_{\rho}}{10^{15}}\right)^{\omega} \cdot\left(\frac{B_{\text {cor }}}{50 \mathrm{G}}\right)^{\beta} . \\
& \cdot\left(\frac{L}{50 \mathrm{Mm}}\right)^{\gamma} \cdot\left(\frac{v_{R M S}}{1.48 \mathrm{~km} / \mathrm{s}}\right)^{\delta} \cdot\left(\frac{\ell}{1 \mathrm{Mm}}\right)^{2.75}\left[\mathrm{~W} / \mathrm{m}^{3}\right]
\end{aligned}
$$

This form was derived by van Wettum et al. (2013), who use it to prescribe the coronal heating in a MHD model. $\ell=1 \mathrm{Mm}$ is here as a constant representing the typical dissipation length scale that has no effect on this scaling law in this case. In contrast to the van Ballegooijen et al. (2011) scaling law, Rappazzo et al. (2008) use the Alfvén crossing time $\tau_{A}$ as time scale and not the correlation time scale $\tau_{0}$ of the driving motions. Because the value range of $\tau_{A}$ reaches to values up to $2000 \mathrm{~s}$, we need to adapt the $\tau$ term of the scaling law so that it represents 


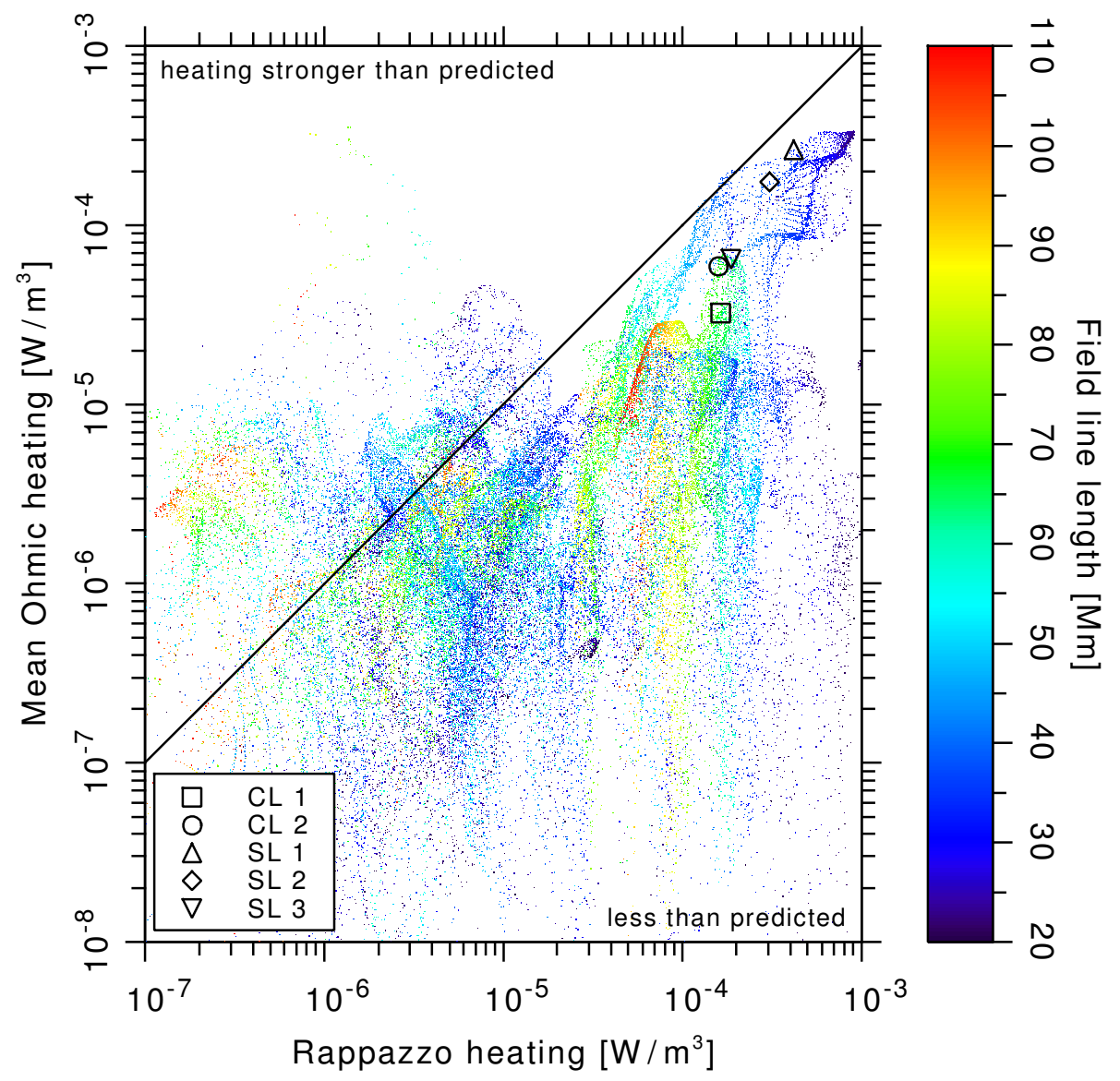

Figure 4.15: Same as Fig. 4.14 but with a modified scaling law with exponents from Rappazzo et al. (2008).

well not only the value range between 60-200s (van Ballegooijen et al. 2011), but also has a continuous effect on larger $\tau$ values. Loops have in our case a length of about $75 \mathrm{Mm}$ that implies $\tau_{A}=750 \mathrm{~s}$ with an Alfvén velocity of about $100 \mathrm{~km} / \mathrm{s}$ in average.

In Fig. 4.15 we see that the Rappazzo et al. (2008) exponents better describe our model data, as compared to the van Ballegooijen et al. (2011) scaling law. The slope of the strongly heated field-lines population (extending towards the upper right in Fig. 4.15) fits better to the equality line (black). Also the discrepancy between the prediction and the model values is much smaller than it is in Fig. 4.14. This shows that the relation between the Ohmic heating to the magnetic field and to the field-line length is stronger in our model than predicted by the van Ballegooijen et al. (2011) scaling law. Even though the Rappazzo et al. (2008) scaling law describes a very similar coronal heating mechanism to the one we use in this work, the predicted coronal heating is still significantly higher than the one in our model. 


\subsubsection{Fitting a new scaling law}

We can of course also try to find better exponents for a purported scaling law of the form:

$$
\begin{aligned}
Q_{\text {ohm }}= & 2.9 \cdot 10^{-3} \cdot \frac{120 \mathrm{~s}}{\tau_{A}+60 \mathrm{~s}} \cdot\left(\frac{n_{\rho}}{10^{15}}\right)^{\omega} \cdot\left(\frac{B_{c o r}}{50 \mathrm{G}}\right)^{\beta} . \\
& \cdot\left(\frac{L}{50 \mathrm{Mm}}\right)^{\gamma} \cdot\left(\frac{v_{R M S}}{1.48 \mathrm{~km} / \mathrm{s}}\right)^{\delta}\left[\mathrm{W} / \mathrm{m}^{3}\right]
\end{aligned}
$$

For this we use a Levenberg-Marquardt (LM) optimization to adjust the exponents $\beta, \gamma$, and $\delta$ in Eqn. 4.3.18. The dependency on the mean particle number density we keep fixed and use the same parameters as in Sect. 4.3.4.2 except that in our model the Alfvén crossing time $\tau_{A}$ through a loop is of the order of 40 minutes, see Sect. 2.3.3,

We exclude the data points with a heating rate lower than the threshold value of $5 \cdot 10^{-6} \mathrm{~W} / \mathrm{m}^{3}$, because there is practically no structure in this data and including it would result in a false impression of accuracy by underestimated error intervals of the fitted exponents. For the fitting, we estimate the error of the model data to a constant value of $25 \%$ of the maximum heating rate. This reflects the large scatter we see in the data and actually puts less weight to the weakly heated field lines. From the LM optimization of the $20^{\prime} 000$ remaining field lines that have a high enough heating rate, we get the exponents

$$
\begin{aligned}
& \beta=1.25 \pm 0.32, \\
& \gamma=-1.65 \pm 0.42, \\
& \delta=1.78 \pm 0.51
\end{aligned}
$$

with a reduced $\chi$ of 2.5 per DOF.

We fixed the parameter $\tau_{A}$ during the optimization, because it acts on the same degree of freedom (DOF) as $\delta$ does, namely shifting the whole data towards higher or lower heating rates. Freeing two parameters for one DOF would result in an underestimated $\chi$ and overestimated errors for the fitted parameters. We keep $\omega=0.125$ as in the Rappazzo et al. (2008) scaling law.

A $\chi$ larger than 1 indicates missing degrees of freedom for the fit or alternatively a too small error estimate, but our error estimate is already quite large. A reduced $\chi$ on the order of 2 , as we find it here, would therefore indicate that there should be roughly double as much DOFs as we give to the fitting procedure. In this case, we can safely state that there are more relations to consider in order to predict the coronal Ohmic heating than just the field-line length, the density, and the magnetic field strength. E.g., it could make a difference if the used footpoint locations of a field line are relatively low or higher up in the atmosphere, because this already 


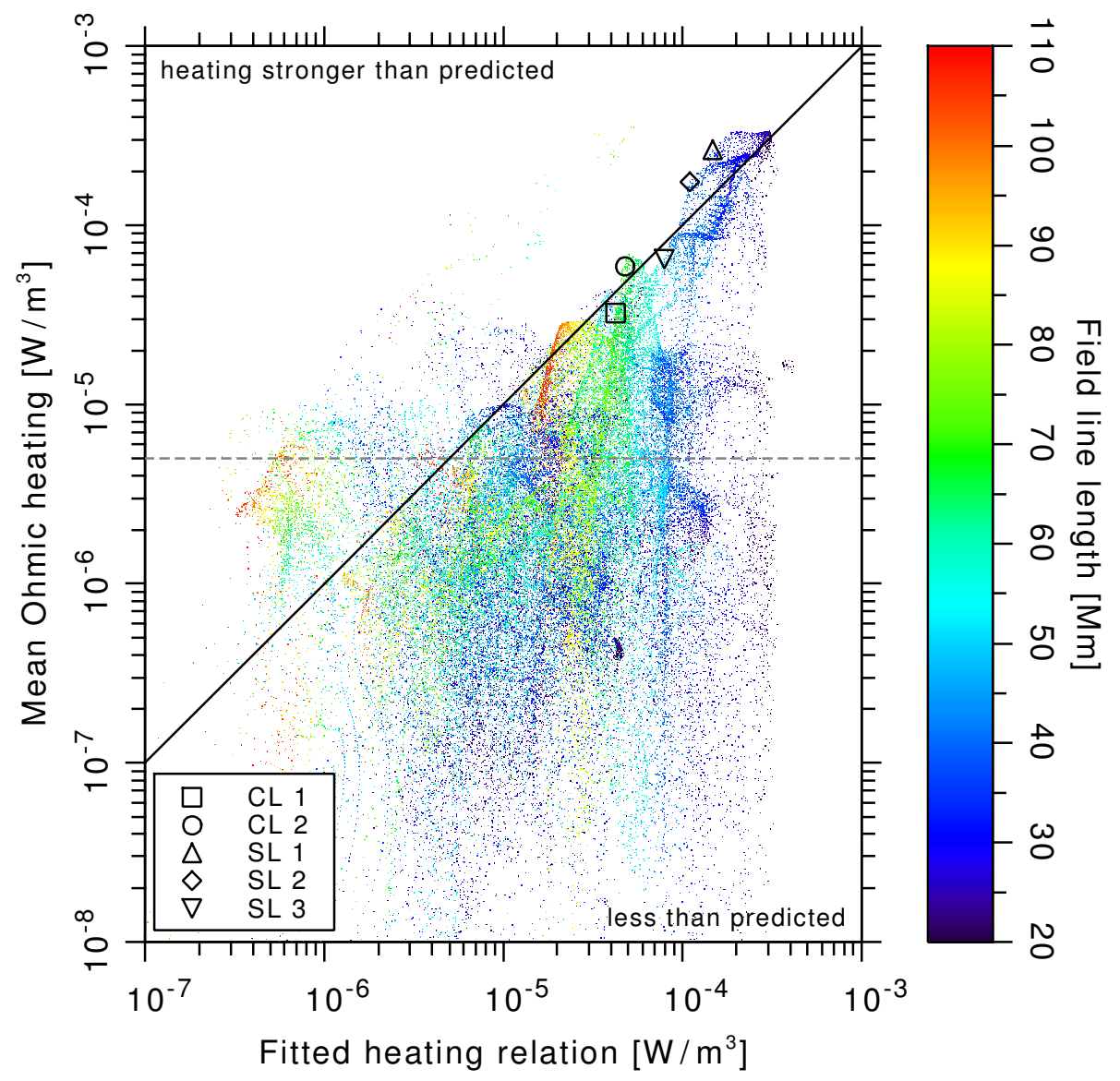

Figure 4.16: Volumetric Ohmic heating as predicted from our fitted scaling law versus the mean Ohmic heating along the model field lines. On the black line the prediction and the model values are equal. The gray dashed line indicates the cutoff value we use for fitting the data points, see Sect. 4.3.4.3.

changes the net Poynting flux into the field line in average (c.f. Fig. 4.1). Also the highly varying net vertical Poynting flux at the field-line footpoints has an influence irrespective of the magnetic field strength.

In Fig. 4.16 we present the scaling law predictions using the exponents as fitted to the data. We find that the population of the strongest heated field lines follows very well the equality line between scaling law and our model heating rate. As the prediction is valid only for the coronal loops with a high dissipation of the energy input, and we know from the previous sections that most of the coronal field lines are not strongly heated, we also see here many field lines with a heating rate significantly lower than predicted (below the equality line).

The dependency of the Ohmic heating to the field-line length, which is stronger in our model data than predicted by van Ballegooijen et al. (2011), reflects, among 
others, the possible geometrical effects due to the curvature of the field in our model. In contrast to this work, the original setup of van Ballegooijen et al. (2011) used coronal loops that are straight structures and hence depend less on the field-line length $L$. A curvature in the magnetic field might amplify the induced coronal currents as compared to a straight loop, because the Ohmic heating is proportional to the currents as $H_{O h m} \sim \vec{j}^{2}$ that have the proportionality $\vec{j} \sim \vec{\nabla} \times \vec{B}$, where the curvature of the magnetic field becomes relevant.

Only the fit of the exponent $\gamma$, representing the dependency on the field-line length, is still consistent within its error as compared to Rappazzo et al. (2008). For the magnetic field strength we find a significantly weaker dependency $\beta$ and the driving motions have in our case a stronger impact on the heating than in Rappazzo et al. (2008).

\subsection{Conclusions}

We found the low density to be a prerequisite for a strong rise of the Ohmic heating per particle and hence the coronal loop-top temperature (c.f. Fig. 4.1). This is interesting, because a lower pressure could on the other hand also be the result of strong heating that expands the plasma adiabatically. But, we see equally strong volumetric Ohmic heating in large parts of our model corona and only where the density is low, the temperature rises later. This gives reasons that in the real corona the volumetric heating is also distributed in space more evenly than the observations of very confined regions with EUV-bright loops would suggest.

The scaling laws (Rosner et al. 1978; Serio et al. 1981; Rappazzo et al. 2008; van Ballegooijen etal. 2011) that we tested against our model field lines indicate only general trends for the most heated and emissive field lines. This puts all attempts to set up scaling laws of the coronal heating into their context, namely that these laws are deduced from and are applicable only on a small subset of coronal loops that are bright in EUV or X-rays.

Regarding the discussion if loops are heated from their footpoints or along the loop, we can say that in our model loops, the volumetric heating is of course stratified following the expansion of the magnetic field (c.f. Fig. 4.1). But, when looking at the heating rates per particle (Fig. 4.2), we see that the heating rate responsible for the high coronal temperatures is actually largest in the corona or close to the loop top. Therefore, speaking of "footpoint heating" (in absolute volumetric values) is physically correct, while it is in the same time irrelevant for basic properties of the coronal loops so that we should better speak of "loop-top heating" (c.f. Priest et al. 1998). 


\section{The transition region Doppler shifts contradiction}

* This chapter is based on a journal article that is in preparation.

\subsection{Motivation}

While on the one hand the heating of the corona is a manifold enigma, on the other hand also the transition region (TR) located between the chromosphere and the lower corona is of high interest, because this interface layer is crucial for the understanding of any mass or energy transport cycles between a Sun-like star and its corona. The reason for this is simply that the corona itself has no independent energy or mass source, so any supplies must pass the transition region. For about 20 years it is known that the TR shows persistent spectral line Doppler displacements (Achour et al. 1995), i.e. redshifts for the cooler TR lines (e.g. CIV), while the hotter lines (e.g. FeX) are blueshifted. This general trend was observed mainly for quiet Sun (QS) regions (Peter and Judge 1999) and also above magnetically active regions (AR) (Teriaca et al. 1999). At first this looks like a contradiction, because upflows and downflows cannot both continuously originate in the same layer of an atmosphere.

In the following, we check for this trend in a 3D MHD model (Sect. 5.2), compare our model data to a possible mechanism in order to explain this trend (Sect. 5.3), and propose a new explanation for it by looking at the plasma flow along individual coronal loops (Sect. 5.4).

\subsection{Doppler-shift statistics}

Using Doppler-shift statistics on the model data, we can check if a general trend of a sign reversal in average Doppler shifts around the TR is also present in our MHD model that has proven to resemble an AR loops system and its plasma flows along these loops (Bourdin et al. 2013). The model was driven by and compared to 
co-temporal observations of the Hinode space observatory (Kosugi et al. 2007; Tsuneta et al. 2008; Culhane et al. 2007).

\subsubsection{Synthetic spectra from MHD model}

From our model, which computes the coronal plasma pressure self-consistently, we synthesize coronal and TR spectra using the atomic database CHIANTI (Dere et al. 1997; Young et al. 2003) following the procedure of Peter et al. (2004 2006). In Fig. 5.1 we show the intensity (left half of each panel) and Doppler line shift maps (right half) of a subvolume around the short loops SL 1-3 in a line-of-sight integration as seen from above (or on disc center). Each intensity map in Fig. 5.1 is for better clarity normalized to its peak intensity. The individual spectral lines are ordered by the line-formation temperature (LFT), where the left column contains spectral lines formed up to $0.1 \mathrm{MK}$, the middle column contains TR lines up to about $0.8 \mathrm{MK}$, and the right column represents the coronal emission at $1 \mathrm{MK}$ and above.

The green contour depicts the boundary of the pixel mask that we derive from the $\mathrm{Fe} X \mathrm{~V}$ intensity with a threshold at one sixth of the peak intensity. We use this mask to select only the pixels that match to observed EUV intensity structures in $\mathrm{Fe} X \mathrm{~V}$, to Doppler line shifts in Fe XII, and to stereoscopic X-ray observations that are well comparable to the FeXV emission from our model (Bourdin et al. 2013). The region close to the mask boundary shows mainly redshifts in the TR, so that our further analysis would just indicate stronger TR redshifts if we enlarge the mask. Furthermore, we do not want to include plasma flows in regions that might not be connected to the coronal loop system and that we might catch when enlarging the mask for the TR lines. Therefore, we stay within this fixed Fe XV mask.

\subsubsection{Spectral properties compared to observations}

Observed spectra of the Sun show a large variation of Doppler shifts and hence broad emission spectra from the TR and the lower corona - typical line widths are in the range of 20-40 km/s (Athay et al. 1983; Peter 2001).

As we are interested in the TR Doppler shifts, we need to find a way to estimate an "effective" Doppler shift of a broad spectrum. "Effective" means here, that we search for a quantity that represents well a large variation in the data and that gives a good estimate for a measurable quantity (like the effective voltage for an amplified current). The spectra we are dealing with usually deviate from Gaussian profiles, because the Doppler shifts and intensities are not normally distributed. 


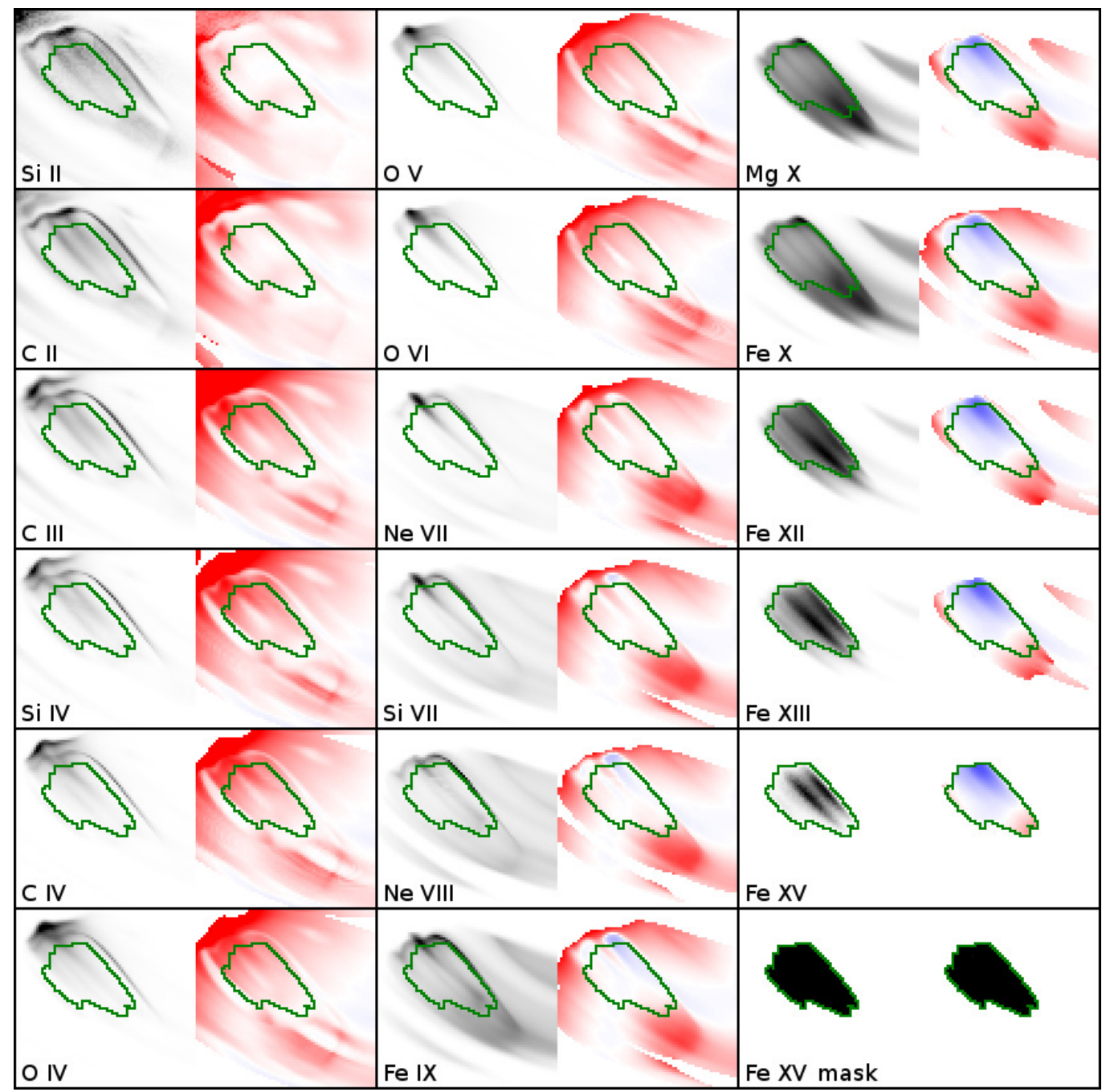

Figure 5.1: Intensity and Doppler-shift maps (in linear scale) for different spectral lines sorted by the LFTs from top to bottom and then from left to right. Depicted here is a snapshot of the FOV containing the small loops SL 1-3, see also Fig. 4.3 and 3.1. The Doppler shfit color code is saturated at $\pm 55 \mathrm{~km} / \mathrm{s}$, which corresponds to the highest redshift of one vertical atmospheric column. A description of the contour line is given in Sect. 5.2.1.

We are mainly interested only in statistical properties of the emission that is integrated along the line-of-sight or also averaged over some field of view (FOV). Such a total spectrum can represent unresolved sub-structure, e.g. within one pixel of a detector or within a spatial or temporal average performed in the observation itself or its post-processing. Furthermore, the addition of a noise background inside an instrument and its subtraction in the post-processing degrades the observation in a way that is not included in our synthetic spectra. Therefore, there are multiple 
possible choices for estimating an effective Doppler shift.

\subsubsection{Estimating an effective Doppler shift}

In order to compare our synthetic Doppler shift maps with observations, we use different "estimators" (in the sense of a statistical estimation function) for an effective Doppler shift produced by the coronal loops system shown in Fig. 5.1 . First, a single-Gaussian fit of the local maximum of a synthetic spectrum points out basic properties of that spectrum by ignoring possible asymmetries in the wings of these spectra.

Second, to compare better with earlier observations and analyses (Peter and Judge 1999: Teriaca et al. 1999), we compute the moment of the total synthetic spectrum as:

$$
\left\langle\lambda_{D}\right\rangle_{\text {moment }}=\int \lambda \cdot I(\lambda) \cdot d \lambda / \int I(\lambda) \cdot d \lambda
$$

with $\lambda$ as the wavelength and $I(\lambda)$ as the respective intensity. This estimator takes the asymmetry in the spectra into account, irrespective of any observational or instrumental degradation effects.

As a third estimator for an effective Doppler shift, we would like to introduce a method that computes a spatial average, weighted by the intensity of each pixel. This method is similar to the moment of a spectrum, but can model quality losses during the measurement, where low intensities would fall into the instrumental noise of a spatial or temporal detector bin and would hardly be detectable, maybe even be suppressed by either instrumental effects or noise reduction methods in the data analysis. Overall, this method gives an estimate of the effective Doppler shift by a thresholded intensity-weighed average (TIWA).

For this method, we define the full FOV and the Fe XV masked pixels as

$$
\begin{aligned}
\vec{r} & \in\{F O V\} \\
\vec{r}_{F e} & \in\left\{\vec{r} \mid I_{F e X V}(\vec{r})>\xi\right\}
\end{aligned}
$$

for the intensity $I_{\Lambda}$ to that we apply a threshold

$$
\xi=1 / 6 \cdot \max \left\{I_{F e X V}(\vec{r})\right\}
$$

at each pixel $\vec{r}$ for a spectral line $\Lambda$. We now define the degraded intensity $\tilde{I}_{\Lambda}$ as

$$
\tilde{I}_{\Lambda}(\vec{r})=\epsilon_{\Lambda}+I_{\Lambda}(\vec{r}) / \max \left\{I_{\Lambda}\left(\vec{r}_{F e}\right)\right\}
$$

with an instrumental noise level of $\epsilon_{\Lambda}$. The effective Doppler shift $\left\langle v_{D}\right\rangle_{\Lambda}$ we estimate for the full FOV as:

$$
\left\langle v_{D}\right\rangle_{\Lambda}(\vec{r})=\frac{\sum v_{D}\left(\vec{r}_{F e}\right) \cdot \tilde{I}_{\Lambda}\left(\vec{r}_{F e}\right)}{\sum \tilde{I}_{\Lambda}\left(\vec{r}_{F e}\right)} \cdot \frac{\#\left\{\vec{r}_{F e}\right\}}{\#\{\vec{r}\}}
$$


An advantage of this TIWA method is that its statistical uncertainty can also be estimated by the variance of the used sample of pixels, which we translate to a standard deviation $\sigma$ of the estimated effective Doppler shift

$$
\sigma_{\Lambda}\left(\left\langle v_{D}\right\rangle_{\Lambda}\right)=\sqrt{\operatorname{Var}\left\{v_{D}\left(\vec{r}_{F e}\right) \cdot \tilde{I}_{\Lambda}\left(\vec{r}_{F e}\right)\right\} \cdot \#_{\text {related }} / \#\left\{\vec{r}_{F e}\right\}}
$$

where the variance is corrected by the number of related pixels in our model. Here, this number is $\#_{\text {related }}=7^{2}$, because in a $6^{\text {th }}$-order numerical scheme 7 pixels in each direction of our 2-dimensional Doppler shift map depend on each other. If not corrected, this would lead to an underestimated variance and hence a false impression of robustness in the TIWA method.

Fourth and finally, when we are blindly dealing with statistical estimators, it is only natural to take the mean of different estimators. This should result in a better estimate, because any methodical effect or systematic error of a single estimator is reduced. In a way, this corresponds to a statistical method called subsampling, because the three estimators use different samples of, or different weights on, the data.

\subsubsection{Properties of selected synthetic spectra}

Now we test the above described estimators with selected synthetic spectra from our model. In Fig. 5.2 we show total spectra of the emission taken from the pixels within the Fe XV mask (see the green contour in Fig. 5.1). The emission from the lower TR ( $\mathrm{Si} I \mathrm{~V}$ is formed around $0.03 \mathrm{MK}$ and C IV at $0.1 \mathrm{MK}$ ) shows roughly a double-Gaussian shape with some extended wings towards high redshifts. Therefore, the three estimators indicate different effective Doppler shifts. We see that the TIWA method (Eqn. 5.2.3) ignores the far-out asymmetric wings that have little intensity, in contrast to the spectral moment that indicates a higher effective redshift.

The emission profile of $\mathrm{OV}$ is more similar to a single-Gaussian with a slight skewness towards redshifts. While the local maximum Gauss fit and the spectral moment lie practically on the maximum intensity, the TIWA method puts more weight on a minority of intense blueshifted pixels, that produce the extended blueshifted wing, and hence predicts a lower effective redshift for $\mathrm{O} V$.

For the FeXV emission, we find roughly a single-Gaussian profile with the line center clearly on the blueshifted side. The MgX spectrum follows a symmetric single-Gaussian profile with a net blueshift of about $-5 \mathrm{~km} / \mathrm{s}$, as indicated by all three estimators. $\mathrm{MgX}$ is formed at lower temperatures than Fe XV and the emission originates in the low corona close to the TR. Therefore, we expect the 


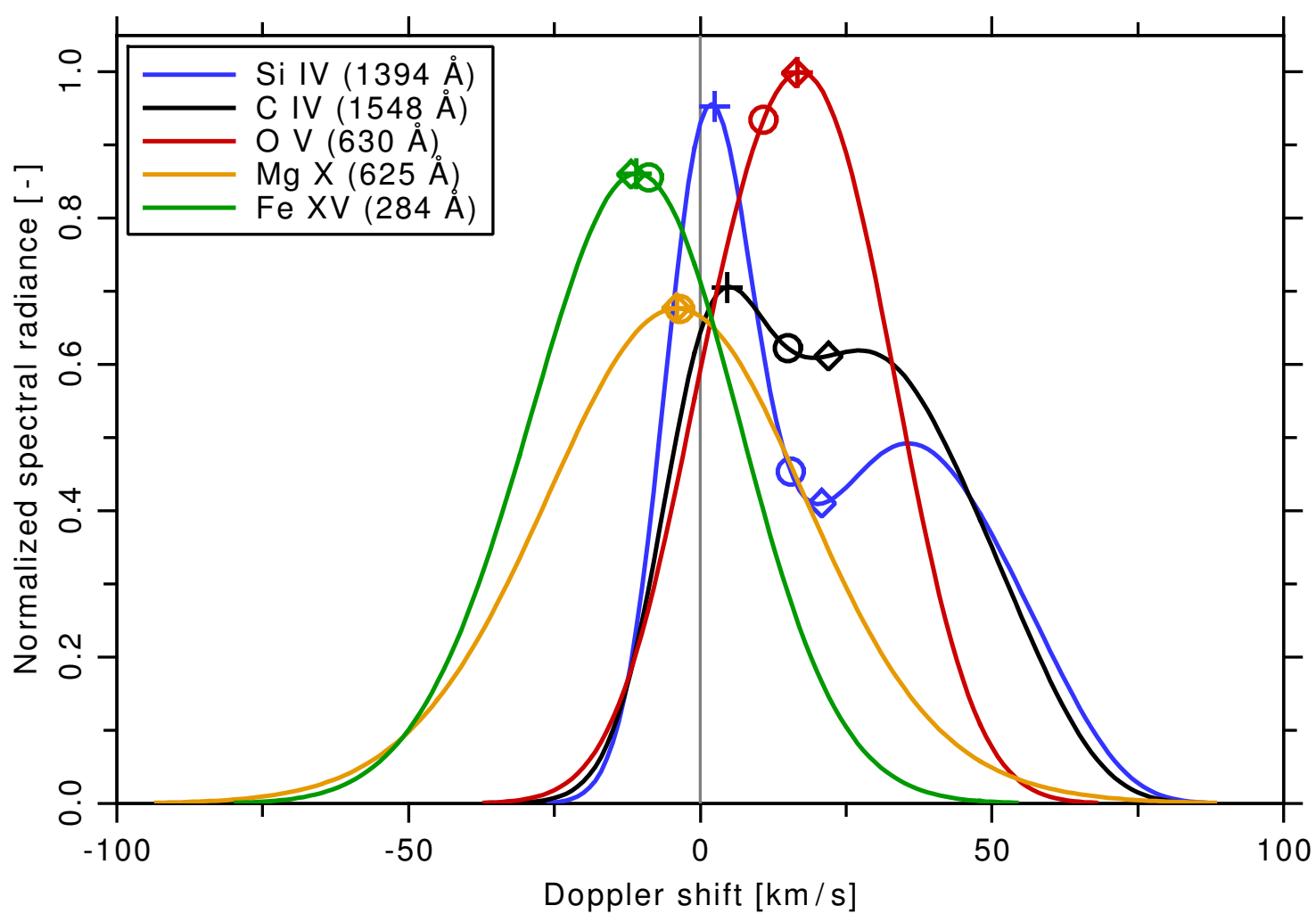

Figure 5.2: Total spectra of five different ions (color coded) representing the TR (SilV, CIV, and $\mathrm{OVI}$ ), as well as EUV emission from the hot corona ( $\mathrm{MgX}$ and $\mathrm{FeXV}$ ). The symbols indicate the effective Doppler shift as estimated by the local maximum (pluses), the TIWA method (circles), and the moment (diamonds), see Sect. 5.2.2.1. The vertical gray line indicates the zero position in Doppler shift, positive values correspond to redshifts, see Sect. 5.2.2.2.

average Doppler shift of $\mathrm{MgX}$ to be closer to zero than for $\mathrm{FeXV}$, because $\mathrm{MgX}$ emission contains both, redshifts and blueshifts evenly distributed in its spectrum.

\subsubsection{Comparing to observations}

In Fig. 5.3 we present two observed average spectra: one of mainly QS (green solid line, Peter and Judge 1999) and one of an AR core (pink dotted line, Teriaca et al. 1999). These lines represent polynomial fits through the obtained data that has a high variance - the vertical velocities in the Solar atmosphere (TR and corona) can vary between $30 \mathrm{~km} / \mathrm{s}$ redshift and $-15 \mathrm{~km} / \mathrm{s}$ blueshift for CIV (c.f. Fig. 5.2 and 5.10 as well as Peter 1999), while the average Doppler shifts are often in the range of $\pm 10 \mathrm{~km} / \mathrm{s}$. 


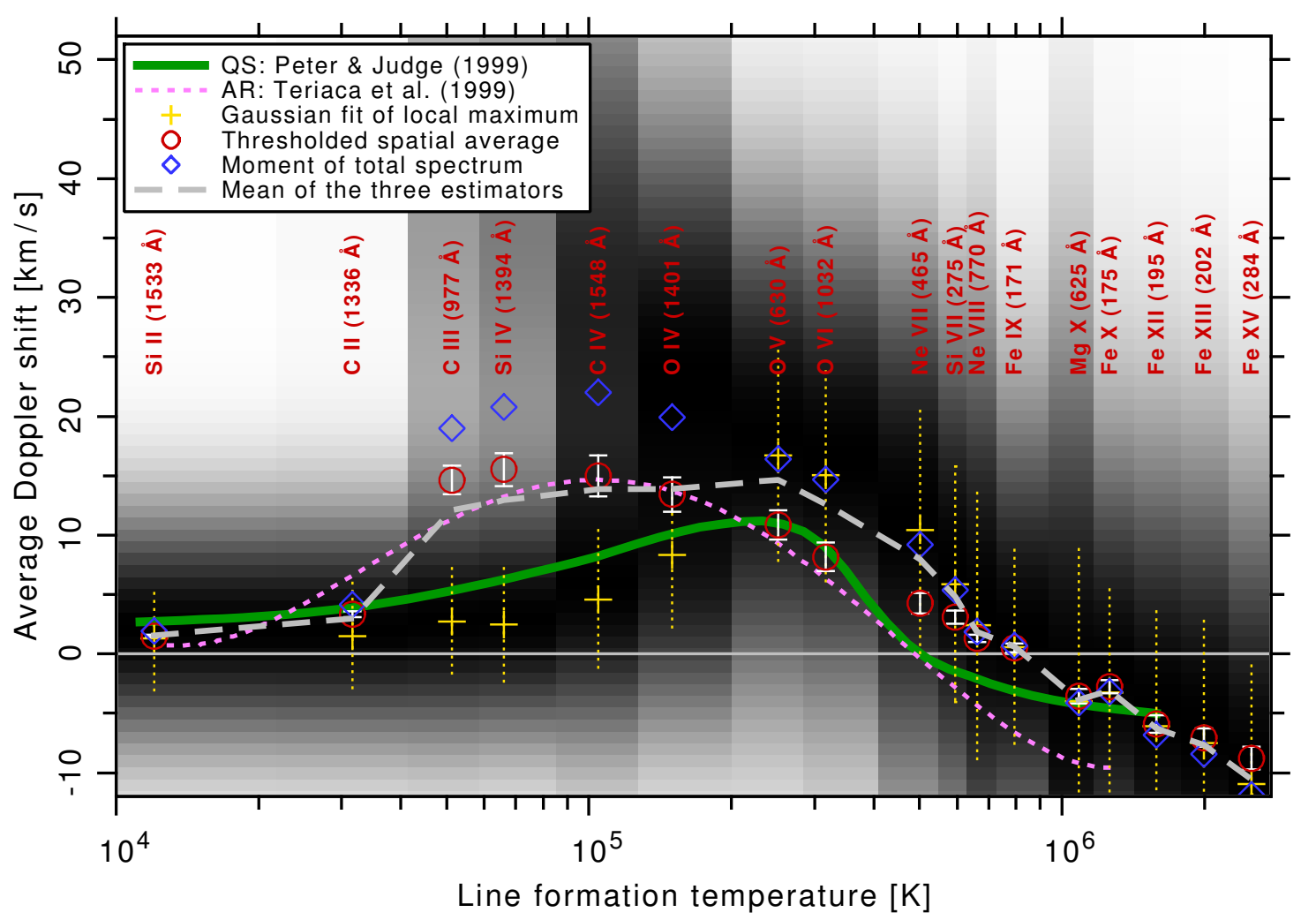

Figure 5.3: Synthetic spectra as derived from our MHD model for different LFTs (c.f. Fig. 5.2), the corresponding ion names are labeled in red. The spectral radiance (in linear grayscale) is normalized to the peak intensity (black). The resolution we use in the spectral direction is $1 \mathrm{~km} / \mathrm{s}$. Positive Doppler shift values indicate redshifts. The line-center positions we get from the Gaussian fit of the local maximum are marked by the orange pluses together with an estimate of the line width (FWHM, length of orange dotted line). The blue diamonds represent the moment of each spectrum. The TIWA estimate of the effective Doppler shift (Eqn. 5.2.3) is indicated with red circles, see Sect. 5.2.2.3 together with the standard deviation (Eqn. 5.2.4) as white bars.

The synthetic total spectra of the Fe XV masked FOV emission are drawn in the background of Fig. 5.3. Some spectra are asymmetric with long-extended redshifted tails (see C III and Si IV), while some other emission lines have roughly a double-Gaussian shape with an extended plateau around the maximum intensity (e.g. CIV and OIV, c.f. Fig. 5.2).

We find that the full-width-half-maximum (FWHM) of the synthetic spectra reach up to about $20 \mathrm{~km} / \mathrm{s}$ for the lines formed above $0.2 \mathrm{MK}$. Here, the Gaussian fit underestimates the FWHM of the total spectrum, because it only reflects the width 
of the main component at the line center. The FWHM of the full spectrum of lines formed above $0.2 \mathrm{MK}$ is about $40 \mathrm{~km} / \mathrm{s}$ (c.f. Fig. 5.2), which is comparable to observed spectra in the same emission lines (Peter 2001).

The Gaussian fit indicates the highest Doppler redshift values roughly at the same LFT of OV at about $0.25 \mathrm{MK}$, as does the QS observation, both indicating redshifts of about $10-15 \mathrm{~km} / \mathrm{s}$.

As to be expected, the spectral moments (blue diamonds in Fig. 5.3) are more sensitive to the long extended redshifted wings of many of the total TR spectra (C III to Si VIII). This results in effective Doppler shifts that are more redshifted than the Gaussian fit, e.g. in the emission lines from C III to Ne VII. In the lines above a LFT of $0.5 \mathrm{MK}$ we find that both methods converge again, because there also the spectra are getting more symmetric as compared to lower LFTs. The general shape of the spectral moments estimator coincides with the AR observations (pink dotted line), while the absolute values have an offset of about $7.5 \mathrm{~km} / \mathrm{s}$ that is still within the uncertainty of the observation (Teriaca et al. 1999).

In the TIWA estimate we take the average of the Doppler shift from pixels selected by the Fe XV mask, weighted by their respective normalized intensity (c.f. red circles in Fig. 5.3). We indicate the uncertainty of this method as a $1 \sigma$ interval with white bars inside the red circles in Fig. 5.3.

Basically, we find that the TIWA method follows the trend that also the two other estimators indicate: significant redshifts in the TR and a turnover point towards blueshifts in the lower corona at about $1 \mathrm{MK}$ in LFT, in particular at the spectral lines of Fe IX and MgX. For the TR the TIWA method lies in between the estimates of the Gaussian fit and the moment of the total spectrum. In the corona above $1 \mathrm{MK}$ the TIWA method has a trend towards a roughly constant blueshift of about $-6 \mathrm{~km} / \mathrm{s}$, which is consistent with the observation of Peter and Judge (1999) that also has this trend towards higher LFTs. In contrast to that, the other two estimators (Gaussian fit and spectral moment) show a trend towards increasing blueshifts for higher LFTs, while these estimates are outside of the $1 \sigma$ interval around the TIWA estimate.

The gray dashed line in Fig. 5.3 indicates the mean of our tree estimators and indeed it reproduces relatively well the overall shape and features of both observations. This mean trend is consistent with the QS data for cool lines ( $\mathrm{Si}$ II and $(I I)$, then mainly follows the AR values up to OIV, and then fits to the QS data again by reproducing the maximum redshifts and the turnover point located around $\mathrm{OV}$ and $\mathrm{OVI}$. Here, it is important to notice that towards higher LFTs, the mean curve continuously turns towards blueshifts also above $1 \mathrm{MK}$, reaching the sign reversal around Fe IX, which was so far only found in observations but could not be reproduced in earlier simulations. The overall shape and slope of the mean 
curve would best fit to the AR data, but shifted towards higher LFTs.

For some cooler TR lines (Si IV, CIV, and OIV) the TIWA method indicates redshifts of about $15 \mathrm{~km} / \mathrm{s}$, which is consistent with the observation of AR loops (Teriaca et al. 1999), while the QS observations are around $5-8 \mathrm{~km} / \mathrm{s}$ (Peter and Judge 1999). With the upcoming IRIS mission, spectra in these emission lines become better accessible and new observations could be made to decide if the bulge of excess red shifts found by the AR observation (in relation to the QS) was only a methodical effect or if these higher redshifts are a fundamental difference for ARs, as our model results would suggest.

\subsection{Heating events as driver of Doppler shifts}

At first, the trend for redshifts below and blueshifts above the TR might look like a contradiction. This trend seems not to be sustainable, because everything that is hot (and supposedly lies above the TR) moves upwards, while everything that is cool (and should lie below the TR) moves downwards. But for example, short heating events could lead to a localized pressure surplus in the atmosphere that then pushes hot material up. Cooler material that is located below such a heating event would then be pushed down. Later, the upwards transported hot material cools down (e.g. due to radiative losses) and flows back downwards into its equilibrium position, while being visible in a lower temperature emission line than while it was pushed upwards. Such a mechanism could (in temporal average) explain the observational findings and was found in radiative ID-MHD simulations of the solar atmosphere (Spadaro et al. 2006) and in a 3D model up to the lower corona (Hansteen et al. 2010).

\subsubsection{Pressure gradient analysis}

The downwards oriented vertical pressure gradient describes an upwards acceleration that is purported to drive the upflows of plasma that was heated in the TR. The maximum of the upwards acceleration should correlate with the blueshifts in an emission line with a LFT of about $1 \mathrm{MK}$ corresponding to the lower corona (e.g. $\mathrm{MgX}$ ). Emission that is formed at temperatures around $0.1 \mathrm{MK}$ in the TR (e.g. CIV) should then show net redshifts.

The maximum upwards and downwards oriented acceleration due to the pressure gradient are:

$$
\begin{aligned}
a_{\text {up }} & =\max \{-\mathrm{d} p / \mathrm{d} s\} / \rho \\
a_{\text {down }} & =\max \{\mathrm{d} p / \mathrm{d} s\} / \rho
\end{aligned}
$$


If the field-aligned pressure gradient $\mathrm{d} p / \mathrm{d} s$ points downwards (towards the photosphere) we give it a negative sign, and if it points upwards (towards the corona) we give it a positive sign.

Hansteen et al. (2010) reported a distribution for $a_{u p}$ that is correlated to redshifts of $2-12 \mathrm{~km} / \mathrm{s}$ in the CIV TR emission line, and at the same time find Doppler shifts for the hotter emission lines, like Si VII formed around $0.6 \mathrm{MK}$ or Fe XII formed around 1.2 MK, that are still consistent with a zero Doppler shift within their respective $1 \sigma$ intervals (see Fig. 11 in Hansteen et al. 2010). The reported values of $a_{u p}$ are obviously not high enough to drive strong upflows against the gravitational acceleration $g$, which otherwise would produce the significant blueshifts one expects in the hotter coronal emission lines. This might be influenced by the size of the simulation domain, which might damp any plasma upflows in the lower corona due to the upper boundary.

\subsubsection{Upwards pressure acceleration}

To compare our model results with Fig. 13 in Hansteen et al. (2010), we applied the same method to Doppler shift maps of the small loops system (SL 1-3). For any data points above a height of $15 \mathrm{Mm}$ we can safely say that they have little to do with the TR. Hence, we do not include them in our analysis here, as they could not be used as a pro argument for the purported mechanism that should be located within the TR or the lower corona.

In Fig. 5.4 we plot $a_{u p}$ as computed from our model data for each vertical atmospheric column versus the average Doppler shift in $\mathrm{MgX}$ of that column. We find a value range for $a_{u p}$ of $4-14 \mathrm{~km} / \mathrm{s}^{2}$, which is several times more than the downwards oriented gravitational acceleration of $g$.

A correlation of $a_{u p}$ to the Doppler blueshifts in $\mathrm{MgX}$ cannot be found. If we use the $\mathrm{Fe} X$ emission line instead, the result is very similar.

\subsubsection{Downwards pressure acceleration}

In Fig. 5.5 we show the maximum acceleration $a_{\text {down }}$ in the opposite direction, that is downwards oriented due to an upwards pressure gradient. Here, we find a value range for $a_{\text {down }}$ of $2-16 \mathrm{~km} / \mathrm{s}^{2}$.

We test for a correlation between the redshifts and the downwards acceleration with an least-absolute-deviations (LAD) fit (red line in Fig. 5.5). The LAD fit, in contrast to a linear least-squares fit, is not so strongly affected by outliers in scattered data which makes the LAD fit more robust.

The fitted slope of $m=1.7 \pm 0.5 \mathrm{~s}$ is positive and the mean absolute deviation of 


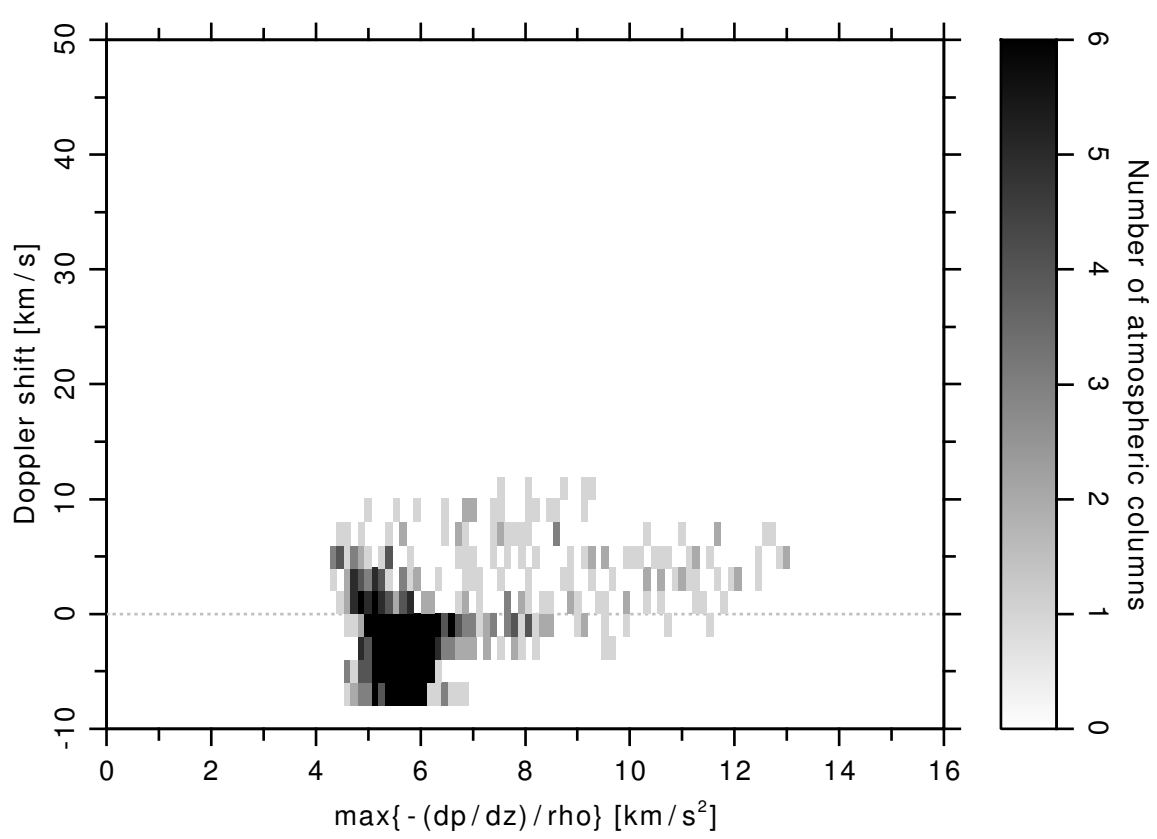

Figure 5.4: Maximum upwards oriented pressure acceleration in each vertical atmospheric column versus the average Doppler shift of the total spectrum of $\mathrm{MgX}$ that is formed in the lower corona. The histogram is built over the atmospheric columns inside the SL 1-3 FOV and selected by the Fe XV mask shown in Fig. 5.1. See Sect. 5.3.2.

the data to the fit is $\sigma=5.7 \mathrm{~km} / \mathrm{s}$ in Doppler shifts. The fit roughly crosses the $\mathrm{y}$-axis at $0.85 \mathrm{~km} / \mathrm{s}$, which is close to the axis origin, as we would expect.

A correlation of $a_{\text {down }}$ to the Doppler redshifts in CIV is not obvious, because there is a large roughly horizontal population at $10 \mathrm{~km} / \mathrm{s}$ in Doppler shifts plus some correlated scatter that broadens towards higher acceleration values. At least, the correlation is significantly less clear as found by Hansteen et al. (2010). Nonetheless, our model data reproduces the persistent TR redshifts and the blueshifts in the lower corona (c.f. Fig. 5.3), therefore the process driving the Doppler shifts must be included in our model description - and if this process is related to the vertical pressure gradient, we should see the correlation here.

\subsubsection{Location of maximum acceleration}

To investigate the effect of the pressure gradient, we show in Fig. 5.6 profiles of the maximum and mean upwards and downwards oriented pressure acceleration versus height. Between heights of 5.5 and $6.5 \mathrm{Mm}$, we find mean upwards accelerations $a_{u p}$ that are larger than the mean gravitational acceleration along the 


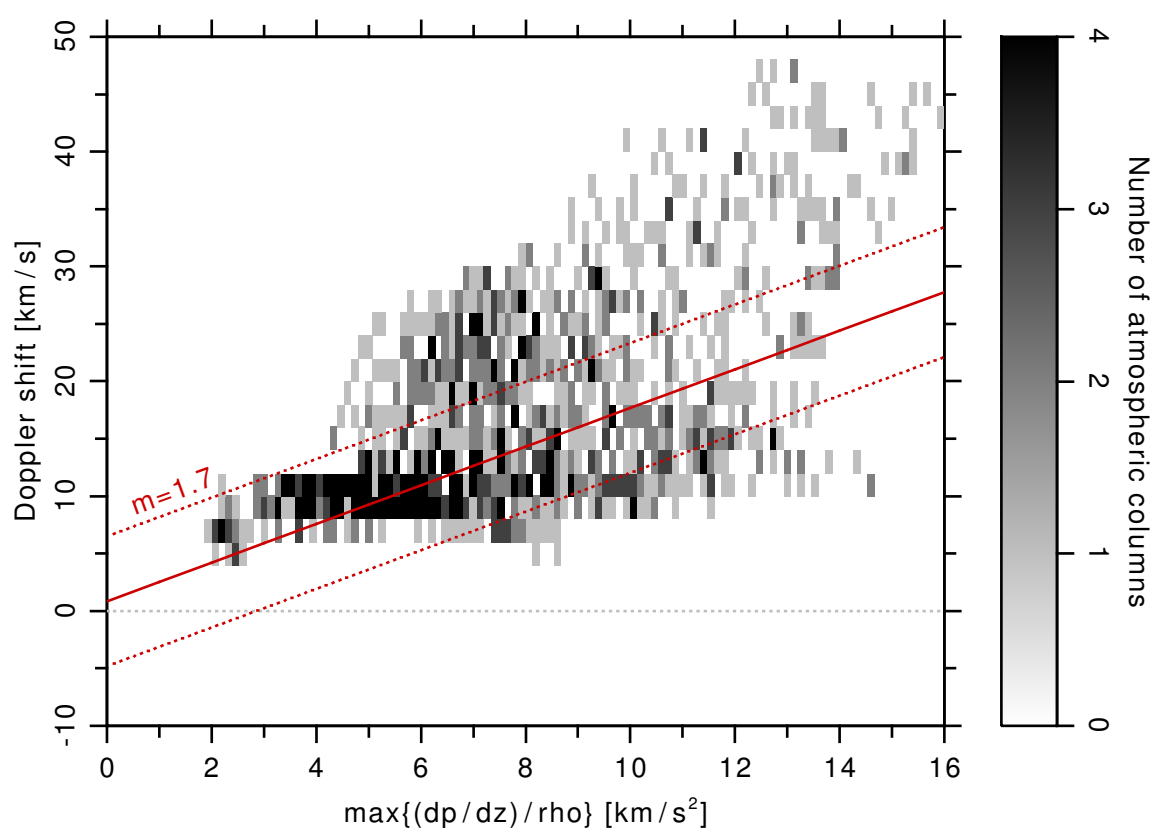

Figure 5.5: Same as Fig. 5.4 but for the maximum downwards oriented pressure acceleration versus the CIV Doppler shifts in an atmospheric column. The red solid line shows an LAD fit with a slope $m$ through the data points and the red dotted line indicates the mean absolute deviation from the fit, see Sect. 5.3.3.

field $g_{\|}$, while above $6.5 \mathrm{Mm} a_{u p}$ is lower than $g_{\|}$that points into the opposite direction. In all heights up to $15 \mathrm{Mm}$ there are some grid volumes in the domain that exceed the gravitational acceleration by a factor of 5 or more, see the maximum upwards acceleration (blue solid line) in Fig. 5.6. which gives reasons for the dynamic plasma flows in the model.

The average (in absolute values) of the pressure gradient $(\mathrm{d} p / \mathrm{d} s) / \rho$ for our whole computational domain is about $59 \mathrm{~m} / \mathrm{s}^{2}$. A vertical pressure acceleration lower than the vertical gravitational acceleration $g=-274 \mathrm{~m} / \mathrm{s}^{2}$ is not in contradiction to a settled atmosphere, because in the corona the plasma beta is smaller than one in large parts of our simulation domain (Gary 2001). This means that also the magnetic field stress is acting as a counter force to gravitation, because horizontal or inclined field keeps the plasma from falling freely in regions where the upwards pressure acceleration is lower than the gravitational acceleration. The average of $g_{\|}$ is lower below $3.5 \mathrm{Mm}$ than above, because the horizontal component of the magnetic field rises quickly when approaching the solar surface. In absolute values our field-aligned pressure acceleration is similar to the global average of the gravitational acceleration along the field $\left\langle g_{\|}\right\rangle=-63 \mathrm{~m} / \mathrm{s}^{2}$. 


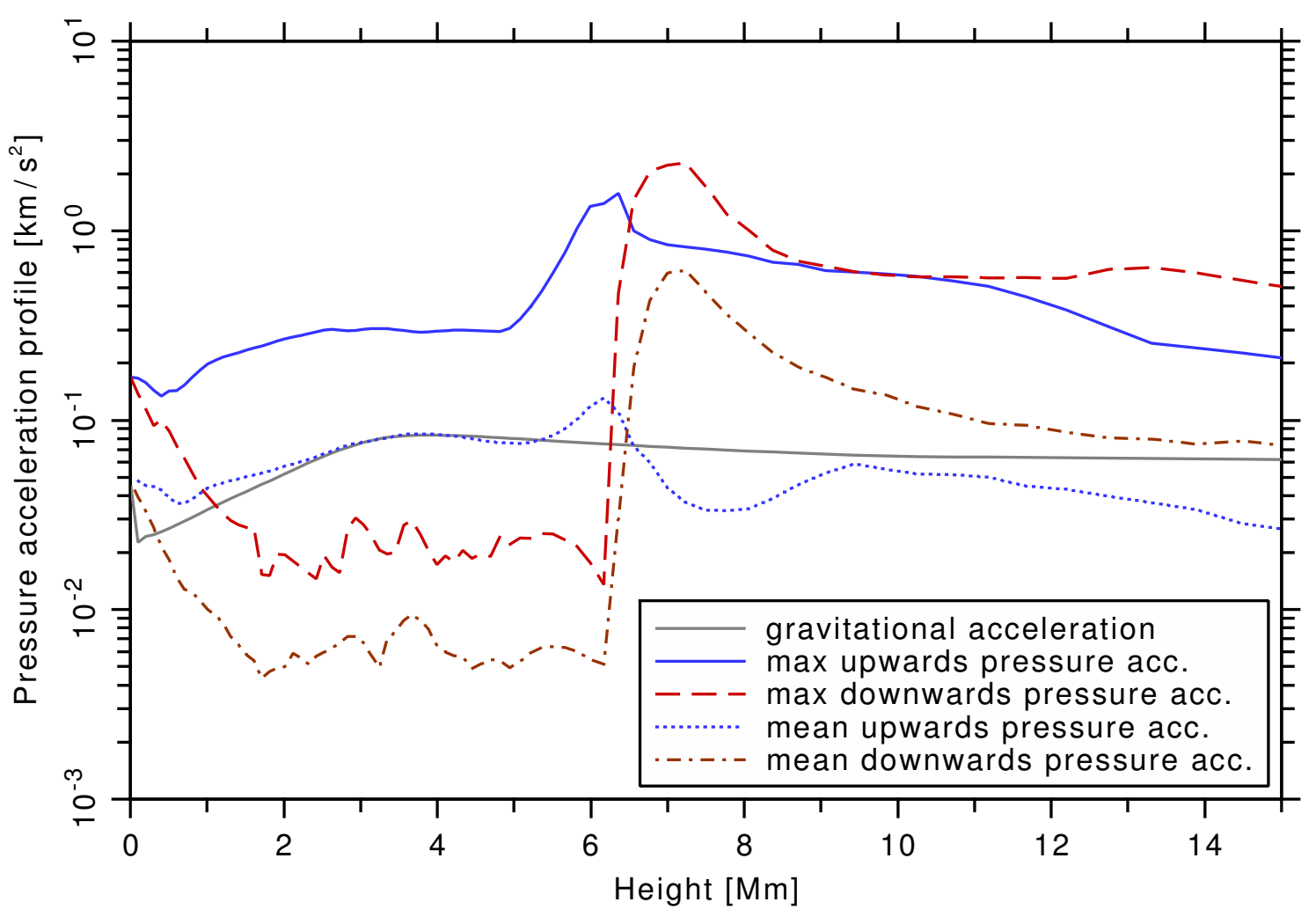

Figure 5.6: Height profile of horizontal averages of the upwards oriented (blue) and the downwards oriented (red) pressure acceleration $a=-(\mathrm{d} p / \mathrm{d} s) / \rho$ due to the pressure gradient along the field $\mathrm{d} p / \mathrm{d} s$. The maximum values (solid and dashed lines) and the mean (dotted and dash-dotted lines) are computed for each height of the SL 1-3 FOV volume over the Fe XV mask (c.f. Fig. 5.1). See Sect. 5.3.4.

We also find that practically everywhere in the TR, that is below $6 \mathrm{Mm}$, the mean upwards pressure acceleration (blue dotted line in Fig. 5.6) either follows or dominates over the downwards oriented pressure acceleration. On the other hand, the mean downwards pressure acceleration clearly dominates above $6 \mathrm{Mm}$.

This means, that the pressure gradient in our model cannot drive TR downflows and in the same time coronal upflows, because the effect of the downwards pressure acceleration will be compensated by the larger upwards acceleration below a height of about $6 \mathrm{Mm}$. Still, the downwards pressure acceleration can have some correlation with the TR redshifts, even though it is located in the lower corona rather than in the TR itself, because downflows that were once accelerated have also to be decelerated by the counteracting TR upwards pressure acceleration. We conclude that the mechanism driving the Doppler shifts in our model is not due to a pressure gradient resulting from short-lived heating events in the TR. 


\subsection{Investigation of prominent individual structures}

As we have all physical quantities available along a coronal loop, we now want to further investigate one loop that can stand representatively for the FOV we analyzed in the Sects. 5.2 and 5.3. Besides the pressure gradient driven plasma flows due to short-lived heating events, an other explanation for the persistent redshifts in the TR would be that different regions can show different steady plasma flows at different temperatures, while a spatial average over these regions could reproduce the observed TR and lower corona Doppler shifts.

In the following we will investigate the properties of the hot coronal loops SL 1 and SL 2 that have formed in our 3D MHD simulation of an AR (c.f. Bourdin et al. 2013). The plasma flow along these loops is persistent in the model already for more than 5 minutes solar time. Therefore, one could not say that this plasma flow is caused by a short-lived heating event.

\subsubsection{Properties of a coronal loop}

\subsubsection{Loop SL 1 investigated}

In Fig. 5.7 we show different physical quantities along the loop SL 1, e.g. the temperature, the density, etc. From the position of the LFTs of CIV $(0.1 \mathrm{MK})$ and of $\mathrm{MgX}(1 \mathrm{MK})$ in each panel (green pluses) we find that the loop is heated very asymmetrically. We find a strong upwards directed net vertical Poynting flux of more than $25 \mathrm{~kW} / \mathrm{m}^{2}$ at the blueshifted upflow side of the loop, that is between 27 and $30 \mathrm{Mm}$ in the distance along the loop. On the downflow side, the Poynting flux reaches only to values around $5-7 \mathrm{~kW} / \mathrm{m}^{2}$, but it is also directed upwards. The strongest Ohmic heating also occurs on the upflow side, which is another indication for the vertical Poynting flux being the energy source for the coronal Ohmic heating, as we have already discussed in the Sects. 4.2.1.3 and 4.3.1.1. We see a strong heating on the upflow side of the loop, which is not the case on the downflow side of the loop. The vertical velocity along the loop shows how the upflow is driven up to the apex of the loop and then turns over to a downflow on the other side. On the upflow side of the loop apex the pressure acceleration is upwards directed and on the downflow side it is downwards directed until it reaches the TR, where we see a sign reversal. Both, vertical velocity along the loop and pressure acceleration indicate a steady continuous siphon flow through the loop. 

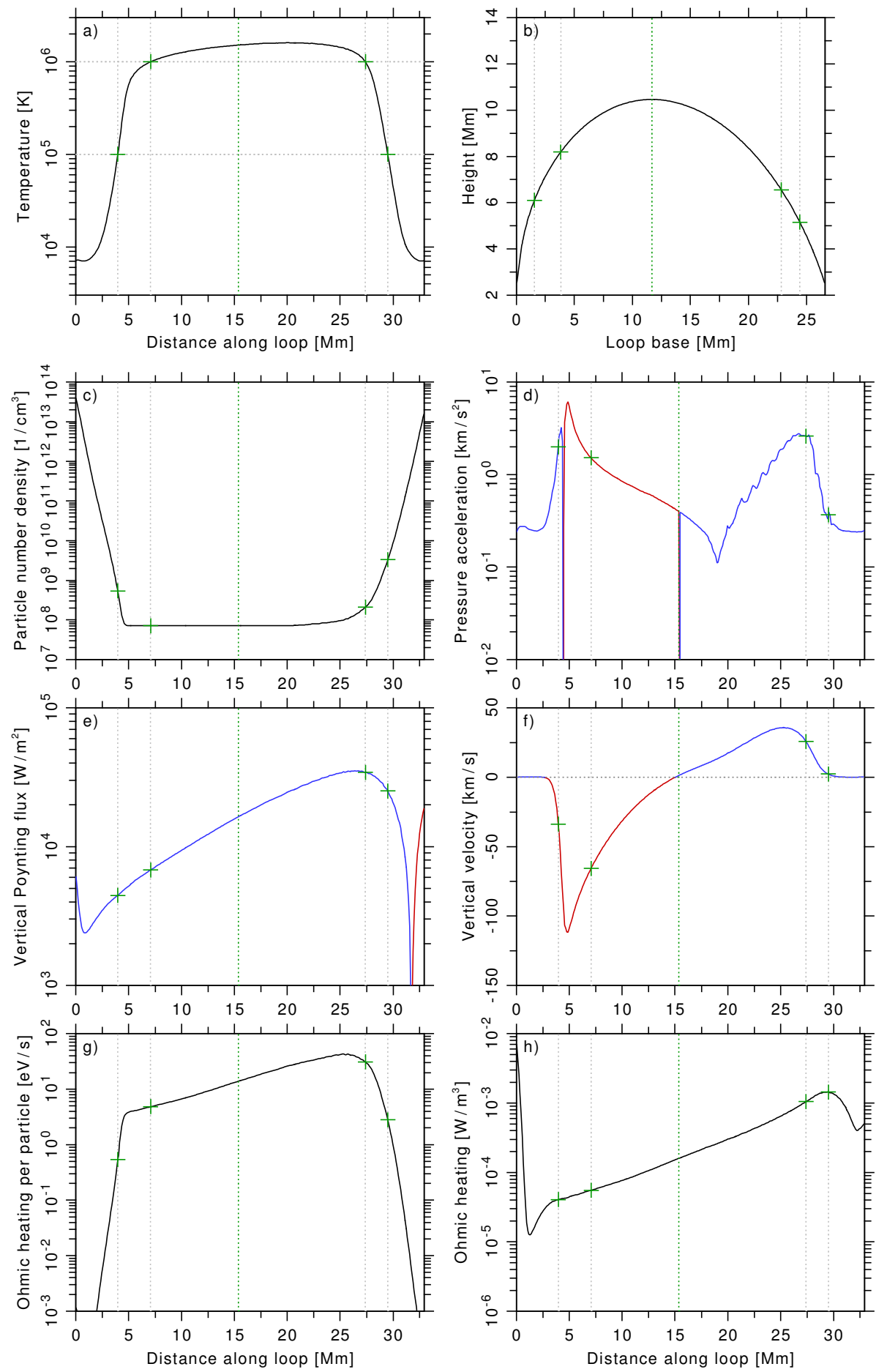

Figure 5.7: Different physical quantities along the loop SL 1 versus the distance along the loop, except for panel b) where we show the height of the loop versus the base of the loop, which is the distance along the loop projected in the vertical direction to the photosphere. The positions where the loop temperature reaches the LFTs of CIV (0.1 MK) and of MgX ( $1 \mathrm{MK})$ are marked by green pluses and vertical gray dotted lines. The loop apex position we indicate as green vertical dotted line. An upwards directed quantity is color coded in blue, while a downwards directed quantity is red. See Sect. 5.4.1.1. 

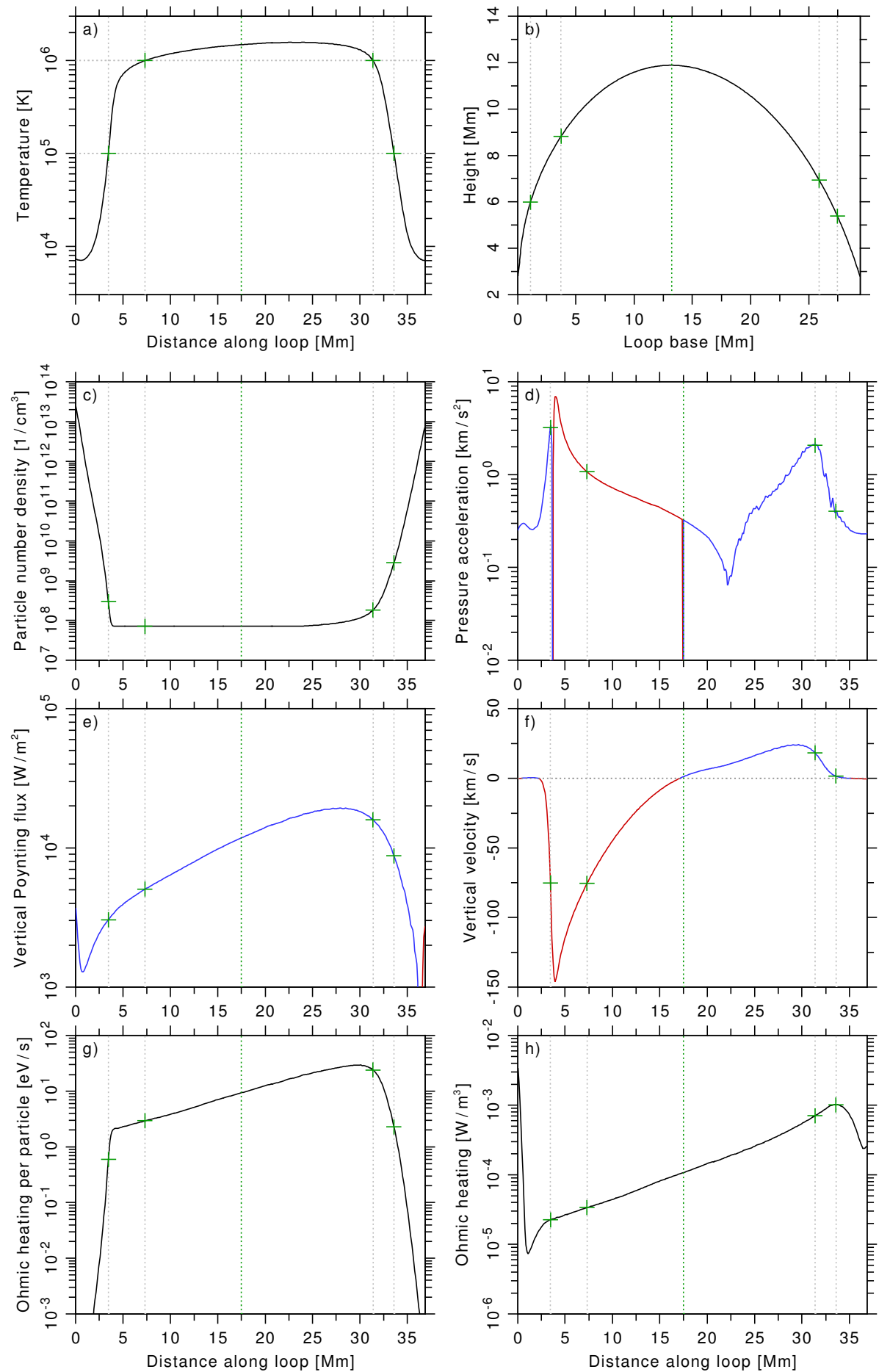

Figure 5.8: Same as Fig. 5.7 but for the loop SL 2, see Sect. 5.4.1.2. The loop base coordinate and the height in panel b) is plotted on the same scale as in Fig. 5.7 so that the loops inclinations can be compared directly. 


\subsubsection{Loop SL 2 investigated}

Besides the findings of Fig. 5.7 we find in Fig. 5.8 that the loop SL 2 is about 1.5 Mm higher than SL 1 and its siphon flow has a lower vertical upflow velocity, while it has a higher vertical downflow velocity. This is not a geometrical effect, because the loops inclinations differ only marginally (c.f. panel b in Fig. 5.7 and 5.8).

On the downflow side, the ratios of the volumetric Ohmic heating at the locations of the CIV and MgX LFTs (vertical gray dotted lines), are rather similar between SL 1 and SL 2. The same is true for the ratios of the Poynting flux. But, the density ratios and in the same way the heating per particle ratios differs significantly between SL 1 and SL 2. These quantities are related to the vertical velocity and the maximum pressure acceleration on the downflow side and this relation should be investigated further with a ID model setup of a coronal loop with as strong asymmetric heating.

\subsubsection{Siphon flow explained}

A steady siphon flow along a coronal loop has been found in earlier ID models (Orlando et al. 1995) and for smaller loops reaching into the TR (Mariska 1988). Orlando et al. (1995) include gravity, radiative losses and a prescribed heating function. Due to the stronger heating on one side, the heat conduction transports energy to the chromosphere, where additional material is evaporated and a pressure surplus is created by adiabatic expansion. As a result, hot material is pushed over the loop apex, cools and falls down on the other side with the help of gravity. The higher density on the upflow side of the loops SL 1 and 2 indicates that a siphon flow is driven here by the stronger heating on the upflow side, that leads to chromospheric evaporation on the upflow side, similar to earlier ID models (Mariska 1988; Orlando et al. 1995).

The absolute value of the Ohmic heating and the Ohmic heating per particle is lower for SL 2 as compared to SL 1. If we compare either at the $1 \mathrm{MK}$ or at the $0.1 \mathrm{MK}$ location (green pluses), we find on the upflow side of SL 2 that the Ohmic heating is about $20 \%$ lower than in SL 1, while on the downflow side it is about $50 \%$ lower. We find a stronger asymmetry in the volumetric Ohmic heating of SL 1 as compared to SL 2.

Besides that, also gravity could be relevant for the higher downflow velocity in SL 2. We see that the maximum downwards pressure acceleration is higher on the downflow side of SL 2 than for SL 1, even though the upwards pressure acceleration on the upflow side is lower than for SL 1. Therefore, also gravity adds to the driving of the downflow in our model loops. 
Still, the higher upwards directed pressure acceleration on the upflow side of SL 1 (and hence the higher upflow velocity in SL 1) is caused by the stronger absolute heating as compared to SL 2 that is leading to more chromospheric evaporation and a pressure surplus on the upflow side of SL 1. Therefore, we identify the asymmetry in the volumetric Ohmic heating as main cause for the siphon flow.

The downflow that we see in both of our model loops SL 1 and 2 is caused by the steady push of material over the loop apex, together with the help of gravity, that is not compensated by the weaker heating on the downflow side.

Both loops SL 1 and 2 have an upwards directed pressure acceleration along the loop at both ends of the loops up to roughly the middle of the TR. On the downflow sides, the CIV emission is even located in an upwards oriented pressure acceleration region, while the $\mathrm{MgX}$ emission is located in a downwards oriented pressure acceleration region, which is the opposite of what Hansteen et al. (2010) have found. Therefore, both of these loops stay in contradiction to the pressure gradient driven mechanism described by Hansteen et al. (2010) to produce downflows in the TR and at the same time upflows in the lower corona. On the other hand, Hansteen et al. (2010) used a model of the QS including some magnetic network rather than an $A R$, so we expect differences to our results.

\subsubsection{Total spetcra for an individual loop}

\subsubsection{Footpoint emission of a loop}

We address now the question, if a strongly asymmetric heating along a loop and the resulting siphon flow could explain the persistent redshifts in the TR and at the same time the blueshifts observed in the lower corona. For that, we draw a "sketch spectrum" created from the sum of the model emission and the Doppler shift along the loop SL 1 originating only from the location where the exact LFTs are reached (c.f. panel $c$ and in Fig. 5.7).

In Fig. 5.9 we show in the left panel the synthetic spectra of CIV exactly at the LFT of $0.1 \mathrm{MK}$ as a sum of the emission from both sides of the loop. One of the Gaussian components of this sum is redshifted and one is blueshifted. The sum of both footpoint emission spectra (black dashed line) has a spectral moment that has a Doppler blueshift of $-1.5 \mathrm{~km} / \mathrm{s}$.

The intensity ratio reflects the emission at both loop sides we get from the model, which is determined by the density ratio, because the emission is taken at the identical LFT on both sides. The intensity of the blueshifted side is much larger than on the redshifted side. This is a selection effect, because we have much more faint redshifted pixels in the CIV emission line than intense blueshifted ones, so that 

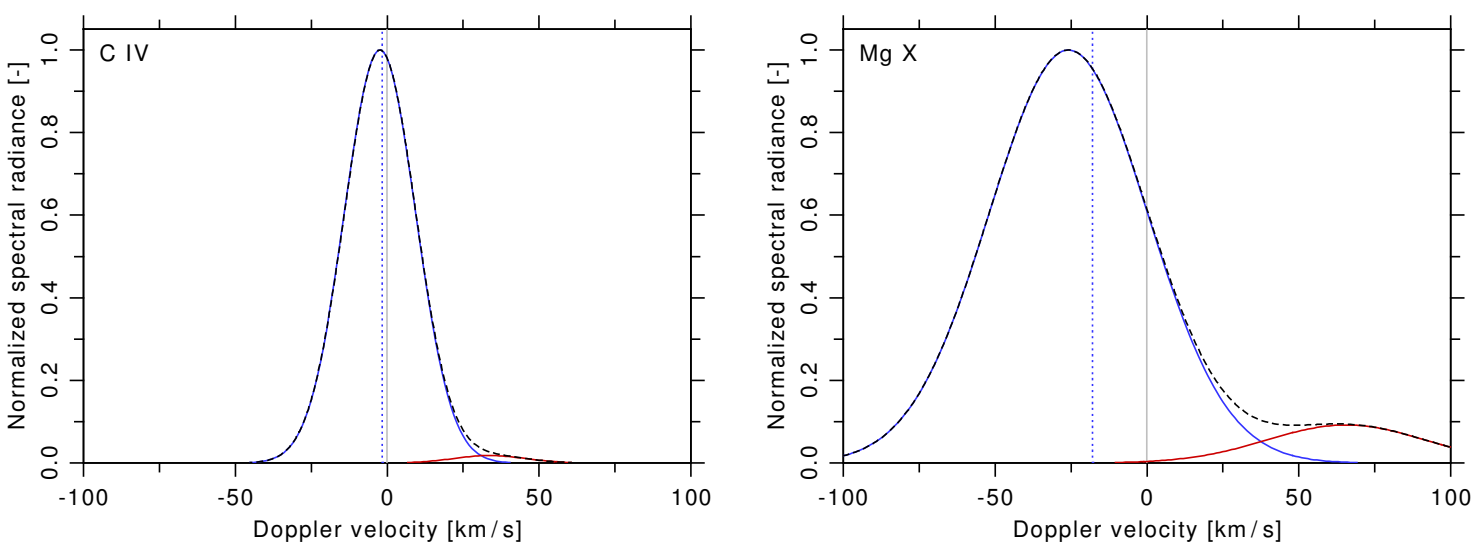

Figure 5.9: Sketch of the sum of the emission created from CIV (left panel) at the TR footpoints of the loop SL 1 and from the $\mathrm{MgX}$ emission (right panel) in the lower corona at $1 \mathrm{MK}$. The blue solid line depicts the Gaussian emission profile corresponding to the upflow side of the loop; the red line represents emission from the downflow side. The sum of both sides spectra (black dashed line) has its line center on the location of the vertical dotted line, where the color is set by the net Doppler shift: red or blue. The vertical gray solid lines indicate the zero position in Doppler shifts. See Sect. 5.4.2.1.

in average the net redshift is stronger than depicted in this sketch and that would lead to a net redshift, if a sum over a larger FOV would be taken (c.f. the CIV in Fig. 5.2).

In the right panel of Fig. 5.9 we plot the synthetic spectra of $\mathrm{MgX}$ at its LFT of $1 \mathrm{MK}$. Here, the total spectrum has a moment that is significantly blueshifted at about $-20 \mathrm{~km} / \mathrm{s}$, which is about twice as much as we would expect from the analysis in Sect. 5.2.2.3.

Obviously, summing the footpoint emission created at only the exact LFT is not sufficient to solve the TR Doppler shifts riddle.

\subsubsection{Integration along a loop}

To see, if the additional emission formed along the loop in C IV would change the result from the previous Sect. 5.9 we now integrate the spectra along the full loop SL 1.

In Fig. 5.10 we show the loop-integrated synthetic C IV spectrum (left panel) that is rather symmetric and of Gaussian shape. The spectral moment (green dashed line) of C IV lies close to the line center and is slightly on the blueshifted side. In contrast to Fig. 5.2 we don't see a second strongly redshifted population forming a second peak and hence no net redshift of the total spectrum. 

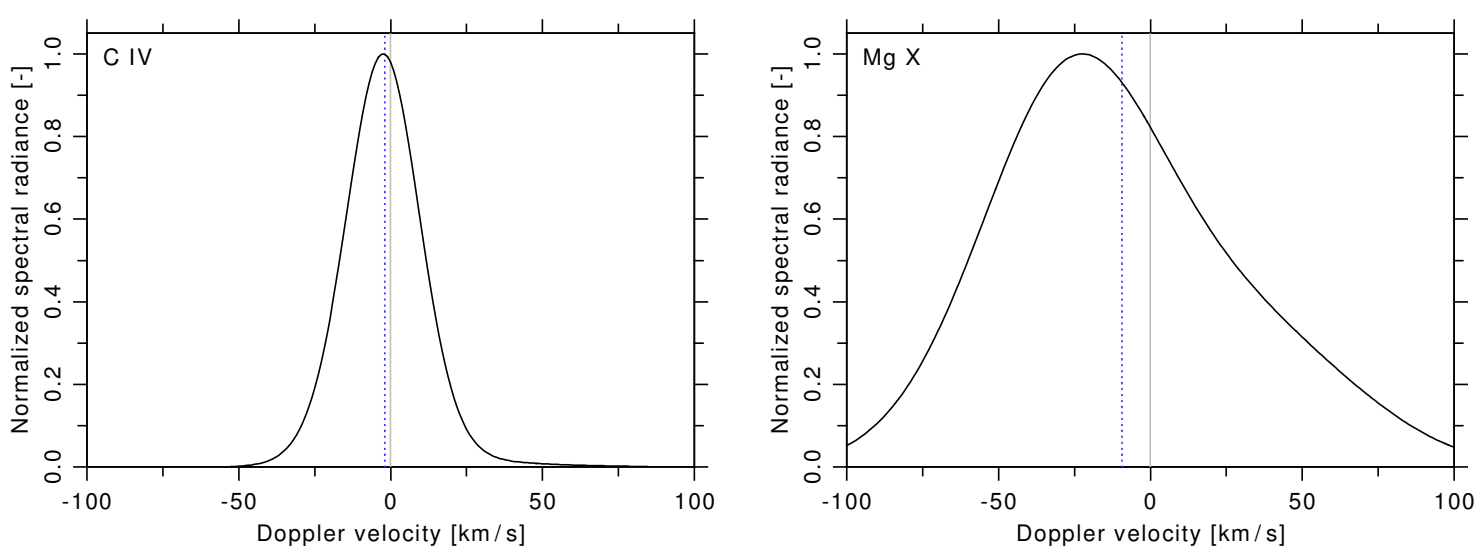

Figure 5.10: Total spectrum along the loop SL 1 of the CIV emission (left panel) and of the MgX emission (right panel). The spectral moment (Eqn. 5.2.1) is marked by the vertical blue dotted line, the vertical gray solid lines indicate the zero position in Doppler shifts, see Sect. 5.4.2.2.

The intensity on the blueshifted side of the loop is much larger than on the redshifted side, as in Sect. 5.4.2.1. Integrating the emission along the loop also has a selection effect, because the faint redshifted pixels in the CIV emission line below or around that loop are not accounted for. In an average over a larger FOV we would find a net redshift, in contrast to this analysis (c.f. Fig. 5.2).

The $\mathrm{MgX}$ spectrum (right panel) is asymmetric with a skewness towards blueshifts and a moment that is significantly blueshifted at about $-10 \mathrm{~km} / \mathrm{s}$, which is what we expect, see also Fig. 5.3. In this case, the net blueshift in the lower corona can be explained already with one sample loop that has a siphon flow.

So, if we extract a ID representation following a magnetic field-line along a loop that emits in EUV, this alone does not explain the net TR redshifts, even if that loop would have the properties of our 3D model loop SL 1, i.e. including the asymmetric heating and the siphon flow.

\subsubsection{Integration of a vertical cut}

In Fig. 5.11 we present the vertical cut through the loop SL 1 as we use it for the following analysis. We see that the main contribution to the total $\mathrm{MgX}$ spectrum comes from the coronal loop spanning between its two footpoints. Also we see that some emission from above (MgX and CIV) and below (CIV) the loop contributes to the total spectrum. This extra emission, that is not accounted for in the Sects. 5.4.2.1 and 5.4.2.2 is the ingredient that turns the net TR Doppler shift towards red, as we show in Fig. 5.12. 


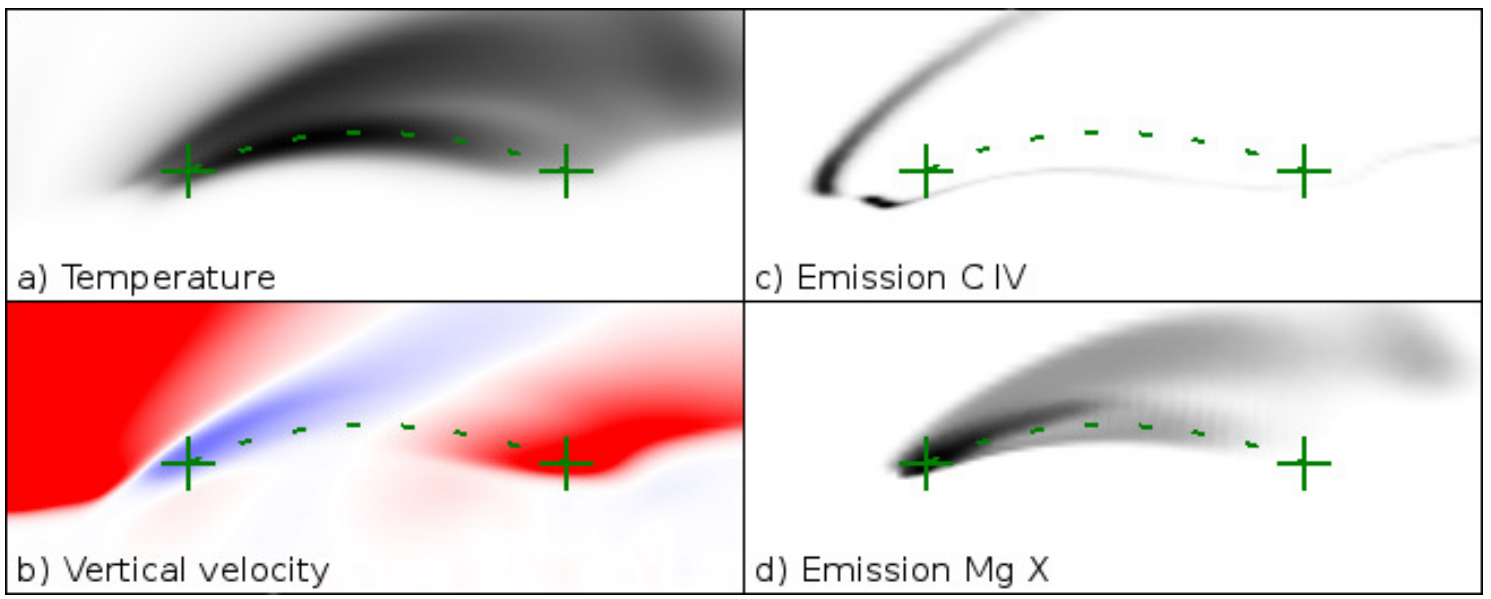

Figure 5.11: Vertical cut through the loop SL 1 showing a) the temperature, b) the vertical velocity, c) the CIV emission, and d) the MgX emission, all in linear scale. The vertical velocity color code is saturated at $\pm 50 \mathrm{~km} / \mathrm{s}$, where upflows are in blue and downflows are in red. We indicate the position of the loop footpoints by the green marks and the loop by green dashes. See Sect. 5.4.2.3.
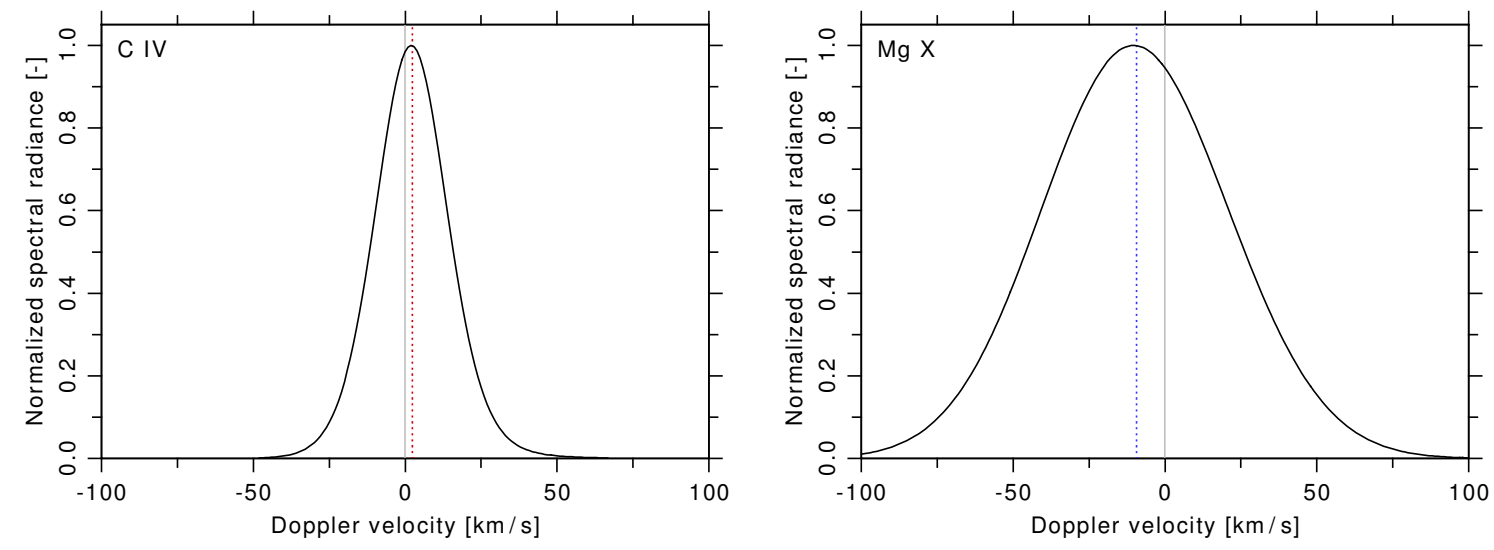

Figure 5.12: Total spectrum of a vertical slice that cuts the loop SL 1. As in Fig. 5.10 we show here the CIV emission (left panel) and the MgX emission (right panel). The spectral moment (Eqn. 5.2.1) is depiced by the vertical dashed line, while the gray line is at the zero Doppler shift position, see Sect. 5.4.2.3.

As we found in Sect. 5.4.2.2 a pure ID view of an asymmetrically heated coronal loop is not enough to reproduce both, TR redshifts and coronal blueshifts at the same time. We now also want to consider emission from above and below that loop, as it would be contained in an observation of that loop from above (or at disc center). The emission from above and below a loop needs to be added both, because the corona is optically thin. 
For $\mathrm{MgX}$ we see now a more symmetric profile with a net blueshift of about $-10 \mathrm{~km} / \mathrm{s}$, as we expect from the AR spectral observations (c.f. Fig. 5.3). The line width and the line symmetry are comparable to the coronal emission formed by Fe XV (c.f. Fig. 5.2).

The total spectrum of such a vertical slice cutting through the loop SL 1 we present in Fig. 5.12. We integrate here the loop's proper emission, as well as some emission above the loop and the complete emission below. We do not include the strong redshifted additional CIV emission visible left above the loop SL 1 (see upper left part of the CIV emission in Fig. 5.11), because it is related to some regional downflow and is not typical, especially it is not related to the loop's proper emission and would only contribute to stronger redshifts, not changing the result, namely net TR redshifts. The emission profile of CIV (left panel) shows now a Gaussian shape with the center slightly redshifted at some $5 \mathrm{~km} / \mathrm{s}$, which is the value we expect from observations.

The net TR redshift is found in the CIV emission line and we can say that the contribution of redshifts mainly from below the loop is necessary to reproduce the TR Doppler shifts as they are observed.

\subsection{Conclusions}

In our model, a strong asymmetric heating drives the siphon flow of the loops SL 1 and 2 (Sect. 5.4.1.3), that explains the observed TR redshifts and coronal blueshifts. The same was found in a ID simulation by Mariska (1988), but there the blueshifts set in already for the OV and O VI emission lines with about $-20 \mathrm{~km} / \mathrm{s}$, which is in contradiction to later observations (c.f. Fig. 5.3). Also in Mariska (1988) the loops were relatively small with an apex height of only $4 \mathrm{Mm}$ and relatively cool with a maximum temperature of $0.5 \mathrm{MK}$, as compared to our model loops SL 1-3. Therefore, in the Mariska (1988) 1D-model hot coronal emission and the corresponding blueshifts could not form, which is now different for this work.

Altogether, we find that a siphon flow in a loop (with properties like SL 2) can explain the persistent TR redshifts and the lower corona blueshifts at the same time by a strongly asymmetric heating profile. The asymmetric Ohmic heating is fed here by an equally asymmetric and upwards directed Poynting flux (Sects. 5.4.1.1 and 5.4.1.2). 


\section{Discussion of the results}

\subsection{Modeling of coronal features}

A sufficient description of the coronal energy balance is crucial to obtain realistic coronal loop structures. The shortest time scales of the relevant processes (i.e. the heat conduction) are on the order of $\mathrm{ms}$. The spatial scales of coronal structures also define the required grid spacing to about $200 \mathrm{~km}$. A self-consistent model of a solar AR in its full spatial extent makes major demands on the theoretical description, as well as on the computational capabilities. Earlier works usually downscaled the domain, used low resolution, prescribed heating functions, or set up advanced ID models (Gudiksen and Nordlund 2002 2005ab; Lionello et al. 2005; Bingert and Peter 2011; Mikić et al. 2013).

The reproduction of features in the corona above an observed $A R$, in particular a hot loops system and the plasma flow dynamics within such loops, is a fundamental step forward in the understanding of the processes that heat and drive the coronal plasma (c.f. Chap. 3). With such data at hand, further analyses become possible that rely on physical quantities that are inaccessible to observations, i.e. the magnetic field configuration in the corona. But already at this stage, the match to observations allows us to say that our model description contains a heating mechanism that delivers energy in the corona at the right places and at the right times. Therefore, the field-line braiding mechanism and the subsequent Ohmic heating due to magnetic diffusion in the corona (Parker 1972) is sufficient to produce hot coronal AR loops.

\subsection{Abstraction of the coronal heating}

With the choice of a constant and uniform magnetic diffusivity $\eta$ we denied pre-knowledge on the distribution of the coronal heat input and, more importantly, we also disregard a possible turbulent cascade and dissipation due to complex microphysical processes. Instead, we model the actual heating mechanism with a simple diffusion equation on the grid scale. It might be surprising that this concept 
gives such realistic results.

An abstraction of the coronal heating can be attempted by fitting scaling laws to either observed coronal loops (Rosner et al. 1978; Serio et al. 1981) or also to field lines extracted from a model (Rappazzo et al. 2008; van Ballegooijen et al. 2011). In Chap. 4 we compare our model data with observational scaling laws that are able to indicate a general trend for hot coronal loops that are bright in EUV and X-rays. But, we also find that there is large variation from these scaling laws, when we take into account a larger sample of model field lines that do not reflect bright coronal loops. We expect this deviation, because even basic properties of faint coronal field lines are practically not observable.

Regarding abstract descriptions of the heating due to reconnection and dissipation of magnetic stress, we find weak similarities in the general trend of an MHD model that braids straight coronal field lines (van Ballegooijen et al. 2011). Mainly we find a significantly stronger dependency of the heating along our model field lines to their lengths and footpoint flux densities. Our model field lines are more similar to Rappazzo et al. (2008) and the exponents for the scaling with length and footpoint flux density reported there are consistent with our own fit of a new scaling law.

\subsection{TR Doppler shifts reproduced}

The riddle of TR redshifts and coronal blueshifts is interesting also regarding the coronal heating, because this interface layer between the chromosphere and the corona reflects the energy and mass cycle with the corona. Therefore, the TR needs to be described well by any model aiming at coronal features that are connected to the lower atmosphere. Like a QS simulation that has a relation between heating events in or above the TR (Hansteen et al. 2010) our AR model also shows such a relation only for the TR redshifts but not for the coronal blueshifts.

Earlier ID models of coronal loops showed that TR redshifts and blueshifts can be driven by an asymmetric heating that leads to a siphon flow through these loops (Mariska 1988; Orlando et al. 1995). In our model we also find siphon flows and the Doppler shift statistics we apply on a small AR loops system indicates the Doppler red- and blueshifts, as they are observed, see Chap. 5. The siphon flows in our model are driven by a surprisingly strong asymmetric Ohmic heating. This is the necessary ingredient to explain the observed TR Doppler shifts enigma. 


\subsection{Outlook}

With this data of young AR loops further analyses can be done. First, it would be interesting to find out about the current density structures we see in the model.

We learned that the Ohmic heating per particle is relevant for the high temperature in the corona, but of course the underlying quantity, the volumetric Ohmic heating is not uniform and so the local density is not the only relevant component in the formation of hot loops. The question remains, whether the current density in the corona is such that it most fits to localized short-lived hating events (Bingert and Peter 2013) following the field-line braiding (nanoflares, see Parker 1972), or if large-scale current sheets form and intersect due to bulk motion or compressional induction in the coronal magnetic field (tectonics, see Priest et al. 2002). The ongoing discussion could be complemented by analyzing the magnetic field topology and searching for quasi-separators.

Second, the temporal evolution of the AR, that has just formed in our model, but was already many solar hours old in the observation, could easily be investigated by simply running the model longer. This we already do and see that the main core loop CL 1 is becoming broader and intenser, and hence becomes more similar to the observed loop. It will be interesting to find out about the stability of the model loops and of the siphon flow in the small loops system.

Third, there is still room for other heating mechanisms to play important roles, e.g. for different coronal features and in particular for the QS corona, because our model does not aim nor reproduce features like plumes, faculae, prominences, flares, or the solar wind acceleration. So, there is still plenty of room (and need) for further modeling of coronal features.

\subsection{Executive summary}

Altogether, we can say that large numerical experiments at the edge of the technical capabilities give insights into physical problems that were otherwise hardly accessible. The use of high-performance computing facilities has an increasing importance for cutting-edge science - not only in the applied sciences, but also for pure fundamental research, like astrophysics. Therefore, large computers are an enabling technique and well-developed programming skills should be an educational goal for natural-sciences students. 



\section{Bibliography}

Achour, H., Brekke, P., Kjeldseth-Moe, O., Maltby, P., 1995, Observed redshifts in the solar transition region above active and quiet regions, ApJ, 453, 945-952

Adams, D., 1979, The Hitchhiker's Guide to the Galaxy, Pan Books/Macmillan

Alfvén, H. H., 1941, On the solar corona, Arkiv för Matematik, Astronomi och Fysik, 27A:25, 1-23

Aschwanden, M. J., 2004, Physics of the Solar Corona. An Introduction, Praxis Publishing Ltd

Aschwanden, M. J., Newmark, J. S., Delaboudinière, J.-P., Neupert, W. M., Klimchuk, J. A., Gary, G. A., Portier-Fozzani, F., Zucker, A., 1999, Three-dimensional Stereoscopic Analysis of Solar Active Region Loops. I. SOHO/EIT Observations at Temperatures of $(1.0-1.5) \cdot 10^{6} \mathrm{~K}, \mathrm{ApJ}, 515,842-867$

Athay, R. G., White, O.R., 1978, Chromospheric and coronal heating by sound waves, ApJ, 226, $1135-1139$

Athay, R. G., Gurman, J. B., Henze, W., Shine, R. A., 1983, Fluid motions in the solar chromosphere-corona transition region. I - Line widths and Doppler shifts for C IV, ApJ, 265, 519-529

Axford, W.I., 1962, The Interaction between the Solar Wind and the Earth's Magnetosphere, J. Geophys. Res., 67, 3791-3796

Babcock, H.W., Babcock, H.D., 1955, The Sun's Magnetic Field, 1952-1954., ApJ, 121,349

Beckers, J. M., Schröter, E.H., 1968, The Intensity, Velocity and Magnetic Structure of a Sunspot Region. I: Observational Technique; Properties of Magnetic Knots, Sol. Phys., 4, 142-164

Berghmans, D., Clette, F., 1999, Active region EUV transient brightenings - First Results by EIT of SOHO JOP80, Sol. Phys., 186, 207-229 
Bingert, S., Peter, H., 2011, Intermittent heating in the solar corona employing a 3D MHD model, A\&A, 530, A 112

Bingert, S., Peter, H., 2013, Nanoflare statistics in an active region 3D MHD coronal model, A\&A, 550, A30

Bingert, S., Zacharias, P., Peter, H., Gudiksen, B., 2010, On the nature of coronal loops above the quiet sun network, Advances in Space Research, 45, 310-313

Bourdin, P.-A., 2011, Denoising observational data, Contributions of the Astronomical Observatory Skalnate Pleso, 41, 149-155

Bourdin, P.-A., Bingert, S., Peter, H., 2013, Observationally driven 3D MHD model of the solar corona above an active region, $A \& A, 555, A 123$

Boyd, T. J. M., Sanderson, J. J., 2003, The Physics of Plasmas, Cambridge University Press

Brandenburg, A., Dobler, W., 2002, Hydromagnetic turbulence in computer simulations, Comp. Phys. Comm., 147, 471-475

Cargill, P. J., Vlahos, L., Baumann, G., Drake, J. F., Nordlund, А̊., 2012, Current Fragmentation and Particle Acceleration in Solar Flares, Space Sci. Rev., 173, $223-245$

Charbonneau, P., 2005, Dynamo Models of the Solar Cycle, Living Reviews in Solar Physics, 2

Cirtain, J. W., Golub, L., Winebarger, A. R., de Pontieu, B., Kobayashi, K., et al., 2013, Energy release in the solar corona from spatially resolved magnetic braids, Nature, 493, 501-503

Cook, J. W., Cheng, C.-C., Jacobs, V. L., Antiochos, S. K., 1989, Effect of coronal elemental abundances on the radiative loss function, ApJ, 338, 1176-1183

Culhane, J. L., Harra, L. K., James, A. M., Al-Janabi, K., Bradley, L. J., Chaudry, R. A., Rees, K., Tandy, J. A., Thomas, P., Whillock, M. C. R., Winter, B., Doschek, G. A., Korendyke, C. M., Brown, C. M., Myers, S., Mariska, J., Seely, J., Lang, J., Kent, B. J., Shaughnessy, B. M., Young, P. R., Simnett, G. M., Castelli, C. M., Mahmoud, S., Mapson-Menard, H., Probyn, B. J., Thomas, R. J., Davila, J., Dere, K., Windt, D., Shea, J., Hagood, R., Moye, R., Hara, H., Watanabe, T., Matsuzaki, K., Kosugi, T., Hansteen, V., Wikstol, Ф., 2007, The EUV Imaging Spectrometer for Hinode, Sol. Phys., 243, 19-61 
De Moortel, I., Ireland, J., Walsh, R. W., Hood, A. W., 2002, Longitudinal intensity oscillations in coronal loops observed with TRACE I. Overview of Measured Parameters, Sol. Phys., 209, 61-88

Dere, K.P., Landi, E., Mason, H. E., Monsignori Fossi, B. C., Young, P. R., 1997 , CHIANTI - An Atomic Database for Emission Lines, A\&AS, 125, 149-173

Edlén, B., 1943, Die Deutung der Emissionslinien im Spektrum der Sonnenkorona. Mit 6 Abbildungen., ZAp, 22, 30-64

Fontenla, J. M., Avrett, E. H., Loeser, R., 1990, Energy Balance in the Solar Transition Region. I. Hydrostatic Thermal Models with Ambipolar Diffusion, ApJ, 355, 700-718

Gary, G. A., 2001, Plasma Beta above a Solar Active Region: Rethinking the Paradigm, Sol. Phys., 203, 71-86

Grotrian, W., 1934, Über das Fraunhofersche Spektrum der Sonnenkorona. Mit 10 Abbildungen., ZAp, 8, 124-146

Gudiksen, B., Nordlund, $\AA$., 2002, Bulk heating and slender magnetic loops in the solar corona, ApJ, 572, L113-116

Gudiksen, B., Nordlund, $\AA$., 2005a, An ab initio approach to the solar coronal heating problem, ApJ, 618, 1020-1030

Gudiksen, B., Nordlund, $\AA$., 2005b, An ab initio approach to solar coronal loops, ApJ, 618, 1031-1038

Hale, G. E., 1908a, On the Probable Existence of a Magnetic Field in Sun-Spots, ApJ, 28, 315-343

Hale, G. E., 1908b, The Zeeman Effect in the Sun, PASP, 20, 287-288

Hansteen, V. H., Hara, H., Pontieu, B.D., Carlsson, M., 2010, On Redshifts and Blueshifts in the Transition Region and Corona, ApJ, 718, 1070-1078

Kamio, S., Hara, H., Watanabe, T., Fredvik, T., Hansteen, V. H., 2010, Modeling of EIS Spectrum Drift from Instrumental Temperatures, Sol. Phys., 266, 209-223

Kippenhahn, R., Möllenhoff, C., 1975, Elementary plasma physics, Mannheim West Germany Bibliographisches Institut AG 
Klimchuk, J. A., 2006, On Solving the Coronal Heating Problem, Sol. Phys., 234, $41-77$

Kosugi, T., Matsuzaki, K., Sakao, T., Shimizu, T., Sone, Y., Tachikawa, S., Hashimoto, T., Minesugi, K., Ohnishi, A., Yamada, T., Tsuneta, S., Hara, H., Ichimoto, K., Suematsu, Y., Shimojo, M., Watanabe, T., Shimada, S., Davis, J. M., Hill, L. D., Owens, J. K., Title, A. M., Culhane, J. L., Harra, L. K., Doschek, G. A., Golub, L., 2007, The Hinode (Solar-B) Mission: An Overview, Sol. Phys., $243,3-17$

Lionello, R., Riley, P., Linker, J. A., Mikić, Z., 2005, The effects of differential rotation on the magnetic structure of the solar corona: Magnetohydrodynamic simulations, ApJ, 625, 463-473

Lyot, B., 1932, Étude de la couronne solaire en dehors des éclipses, ZAp, 5, 73-95

Mariska, J. T., 1988, Observational signatures of loop flows driven by asymmetric heating, ApJ, 334, 489-493

McIntosh, S. W., de Pontieu, B., Carlsson, M., Hansteen, V., Boerner, P., Goossens, M., 2011, Alfvénic waves with sufficient energy to power the quiet solar corona and fast solar wind, Nature, 475, 477-480

Mikić, Z., Lionello, R., Mok, Y., Linker, J. A., Winebarger, A. R., 2013, The Importance of Geometric Effects in Coronal Loop Models, ApJ, 773, 94

Moore, R. L., Suess, S. T., Musielak, Z. E., An, C.-H., 1991, Alfven wave trapping, network microflaring, and heating in solar coronal holes, ApJ, 378, 347-359

Narain, U., Ulmschneider, P., 1996, Chromospheric and Coronal Heating Mechanisms II, Space Sci. Rev., 75, 453-509

November, L. J., Koutchmy, S., 1996, White-Light Coronal Dark Threads and Density Fine Structure, ApJ, 466, 512-528

Orlando, S., Peres, G., Serio, S., 1995, Models of stationary siphon flows in stratified, thermally conducting coronal loops. 1: Regular solutions, A\&A, 294, $861-873$

Parker, E. N., 1963, Interplanetary dynamical processes, New York, Interscience Publishers 
Parker, E. N., 1972, Topological Dissipation and the Small-Scale Fields in Turbulent Gases, ApJ, 174, 499-510

Parker, E. N., 1988, Nanoflares and the solar X-ray corona, ApJ, 330, 474-479

Parker, E. N., 1983, Magnetic Neutral Sheets in Evolving Fields - Part Two Formation of the Solar Corona, ApJ, 264, 642-647

Patsourakos, S., Klimchuk, J. A., 2005, Coronal Loop Heating by Nanoflares: The Impact of the Field-aligned Distribution of the Heating on Loop Observations, ApJ, 628, 1023-1030

Peter, H., 1999, Analysis of transition-region emission line profiles from full-disk scans of the Sun using the SUMER instrument on SOHO, ApJ, 516, 490-504

Peter, $\mathrm{H}$., 2001, On the nature of the transition region from the chromosphere to the corona of the Sun, $A \& A, 374,1108-1120$

Peter, H., Bingert, S., 2012, Constant cross section of loops in the solar corona, $A \& A, 548, A 1$

Peter, H., Judge, P. G., 1999, On the Doppler shifts of solar UV emission lines, ApJ, 522, 1148-1166

Peter, H., Gudiksen, B., Nordlund, ^., 2004, Coronal heating through braiding of magnetic field lines, ApJ, 617, L85-L88

Peter, H., Gudiksen, B., Nordlund, A., 2006, Forward modeling of the corona of the sun and solar-like stars: from a three-dimensional magnetohydrodynamic model to synthetic extreme-ultraviolet spectra, ApJ, 638, 1086-1100

Porter, J. G., Klimchuk, J. A., 1995, Soft X-Ray Loops and Coronal Heating, ApJ, 454, 499-511

Priest, E. R., 1982, Solar Magnetohydrodynamics, D. Reidel, Dordrecht

Priest, E. R., Foley, C. R., Heyvaerts, J., Arber, T.D., Culhane, J. L., Acton, L. W., 1998, Nature of the heating mechanism for the diffuse solar corona, Nature, 393, 545-547

Priest, E. R., Heyvaerts, J. F., Title, A. M., 2002, A Flux-Tube Tectonics Model for Solar Coronal Heating Driven by the Magnetic Carpet, ApJ, 576, 533-551

Rappazzo, A. F., Velli, M., Einaudi, G., Dahlburg, R. B., 2008, Nonlinear Dynamics of the Parker Scenario for Coronal Heating, ApJ, 677, 1348-1366 
Rosner, R., Tucker, W.H., Vaiana, G.S., 1978, Dynamics of the quiescent solar corona, ApJ, 220, 643-665

Schwenn, R., 2006, Space Weather: The Solar Perspective, Living Reviews in Solar Physics, 3, 2

Serio, S., Peres, G., Vaiana, G. S., Golub, L., Rosner, R., 1981, Closed coronal structures. II - Generalized hydrostatic model, ApJ, 243, 288-300

Solanki, S. K., Lagg, A., Woch, J., Krupp, N., Collados, M., 2003, Three-dimensional magnetic field topology in a region of solar coronal heating, Nature, 425, 692-695

Spadaro, D., Lanza, A. F., Karpen, J. T., Antiochos, S. K., 2006, A Transient Heating Model for the Structure and Dynamics of the Solar Transition Region, ApJ, 642, 579-583

Spitzer, L., 1962, Physics of Fully lonized Gases, Interscience, New York (2nd edition)

Stix, M., 2004, The Sun. An introduction, Springer, Berlin (2nd edition, corrected 2nd printing)

Strong, K., Bruner, M., Tarbell, T., Title, A., Wolfson, C. J., 1994, Trace - The transition region and coronal explorer, Space Sci. Rev., 70, 119-122

Sturrock, P. A., 1999, Chromospheric Magnetic Reconnection and Its Possible Relationship to Coronal Heating, ApJ, 521, 451-459

Teriaca, L., Banerjee, D., Doyle, J. G., 1999, SUMER observations of Doppler shift in the quiet Sun and in an active region, $A \& A, 349,636-648$

Tsuneta, S., Ichimoto, K., Katsukawa, Y., Nagata, S., Otsubo, M., Shimizu, T., Suematsu, Y., Nakagiri, M., Noguchi, M., Tarbell, T., Title, A., Shine, R., Rosenberg, W., Hoffmann, C., Jurcevich, B., Kushner, G., Levay, M., Lites, B., Elmore, D., Matsushita, T., Kawaguchi, N., Saito, H., Mikami, I., Hill, L.D., Owens, J. K., 2008, The Solar Optical Telescope for the Hinode Mission: An Overview, Sol. Phys., 249, 167-196

Unsöld, A., Baschek, B., 2002, Der neue Kosmos. Einführung in die Astronomie und Astrophysik, Springer, Berlin (7. Auflage), ISBN ISBN 3-540-42177-7

Švestka, Z., 1956, Several Notes on the Statistics of Chromosferic Flares, Bulletin of the Astronomical Institutes of Czechoslovakia, 7, 9-17 
van Ballegooijen, A. A., Asgari-Targhi, M., Cranmer, S. R., DeLuca, E. E., 2011 , Heating of the Solar Chromosphere and Corona by Alfvén Wave Turbulence, ApJ, 736,3

van Wettum, T., Bingert, S., Peter, H., 2013, Parameterisation of coronal heating: spatial distribution and observable consequences, A\&A, 554, A39

Wedemeyer-Böhm, S., Scullion, E., Steiner, O., Rouppe van der Voort, L., de La Cruz Rodriguez, J., Fedun, V., Erdélyi, R., 2012, Magnetic tornadoes as energy channels into the solar corona, Nature, 486, 505-508

Withbroe, G. L., 1988, The temperature structure, mass, and energy flow in the corona and inner solar wind, ApJ, 325, 442-467

Withbroe, G. L., Noyes, R. W., 1977, Mass and energy flow in the solar chromosphere and corona, ARA\&A, 15, 363-387

Young, P. R., Del Zanna, G., Landi, E., Dere, K. P., Mason, H. E., Landini, M., 2003, CHIANTI - an atomic database for emission lines. VI. Proton Rates and Other Improvements, ApJS, 144, 135-152

Zacharias, P., Peter, H., Bingert, S., 2011 a, Investigation of mass flows in the transition region and corona in a three-dimensional numerical model approach, A\&A, 531, A97+

Zacharias, P., Peter, H., Bingert, S., $2011 \mathrm{~b}$, Ejection of cool plasma into the hot corona, A\&A, 532, A112+ 



\section{A Appendix}

\section{A.1 Serio correction factor unraveled}

We expand here on the correction factor $E_{T}$ to the RTV scaling law introduced by Serio et al. (1981), that leads to a better fit between the predicted and our model loop parameters, as shown in Fig. 4.11 and 4.12 see Sect. 4.3.3.2. This correction factor is applied to the estimation of the loop-top temperature $T_{\max }$, see Eqns. 4.3 .3 and 4.3.14. The heating scale height $s_{H}$ we estimated to $7.5 \mathrm{Mm}$ from our model data. The pressure scale height is $s_{P}=R T /(\mu g)$, where $\mu$ is the molar mass, $R$ is the universal gas constant, and $g$ is the gravitational acceleration. We estimated $s_{P}$ to $50 \mathrm{Mm}$ and used it as a constant for this analysis.

The RTV scaling law can be modified so that the volumetric Ohmic heating integrated along the coronal part of a field line $F_{\text {Ohm }}$ relates to the maximum temperature $T_{\max } \sim F_{\text {Ohm }}{ }^{2 / 7} L^{2 / 7} \cdot E_{T}$, see Eqn. 4.3.11. The modified scaling law splits up into a relation between the temperature and either the Ohmic heating, loop length, or the correction factor only.

In Fig. A. 1 we show the relation between the RTV temperature and the field-line length $L^{2 / 7}$, excluding the Serio et al. (1981) correction factor $E_{T}$ and the dependency on the Ohmic heating $F_{\text {Ohm }}$. The value range of the length relation is relatively narrow, as it spans only over a relative factor of 1.6 between the longest and the shortest field lines used for our analysis. Basically, this relation itself doesn't give the whole distribution much structure, because its impact on the maximum temperature is weak.

In Fig. A.2 we plot the temperature versus only the Serio correction factor $E_{T}$. The correction factor $E_{T}$ alone reaches to relative factors of 2.8 between the longest and the shortest field lines. The dependence on the loop length is rather linear, as can be read from the color code. As for the length relation (c.f. Fig. A.1) this correction factor wouldn't give the distribution much additional structure. To check the importance of the length relation together with the correction factor $L^{2 / 7} \cdot E_{T}$, we plot this quantity in Fig. A.3. Both parts of the Serio scaling law partly cancel each other, as we find here a maximum relative factor of 1.8 , which 


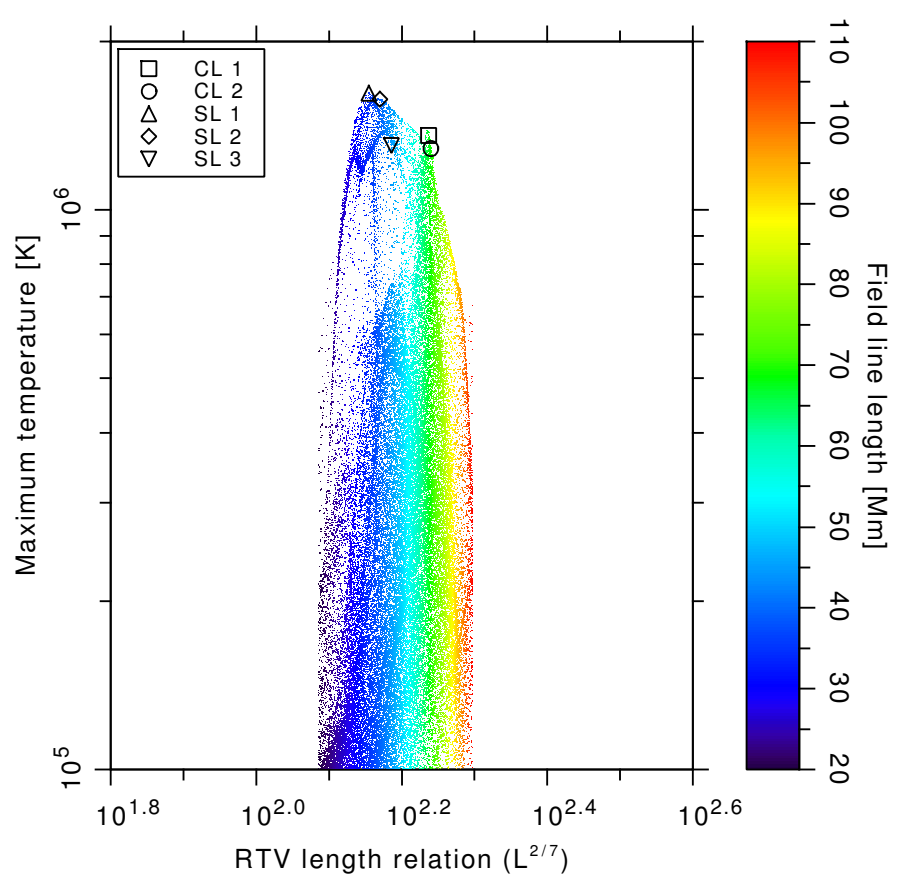

Figure A. 1: RTV temperature (length relation) versus maximum temperature along a field line.

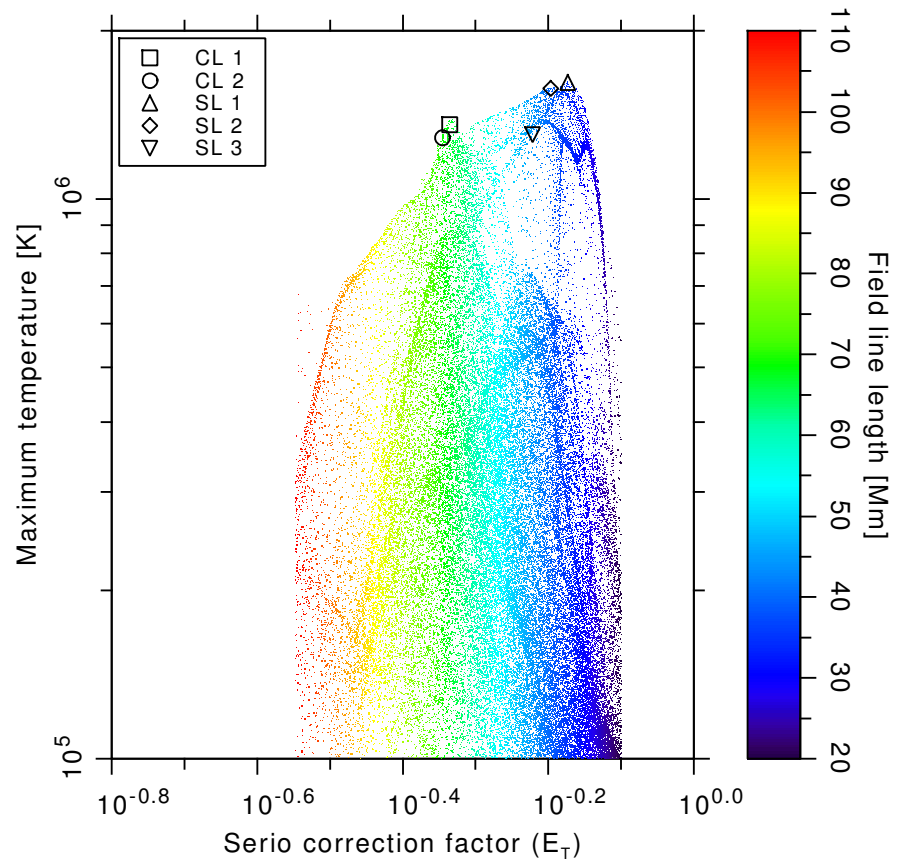

Figure A.2: Serio-RTV temperature (correction factor relation) versus maximum temperature along a field line. 


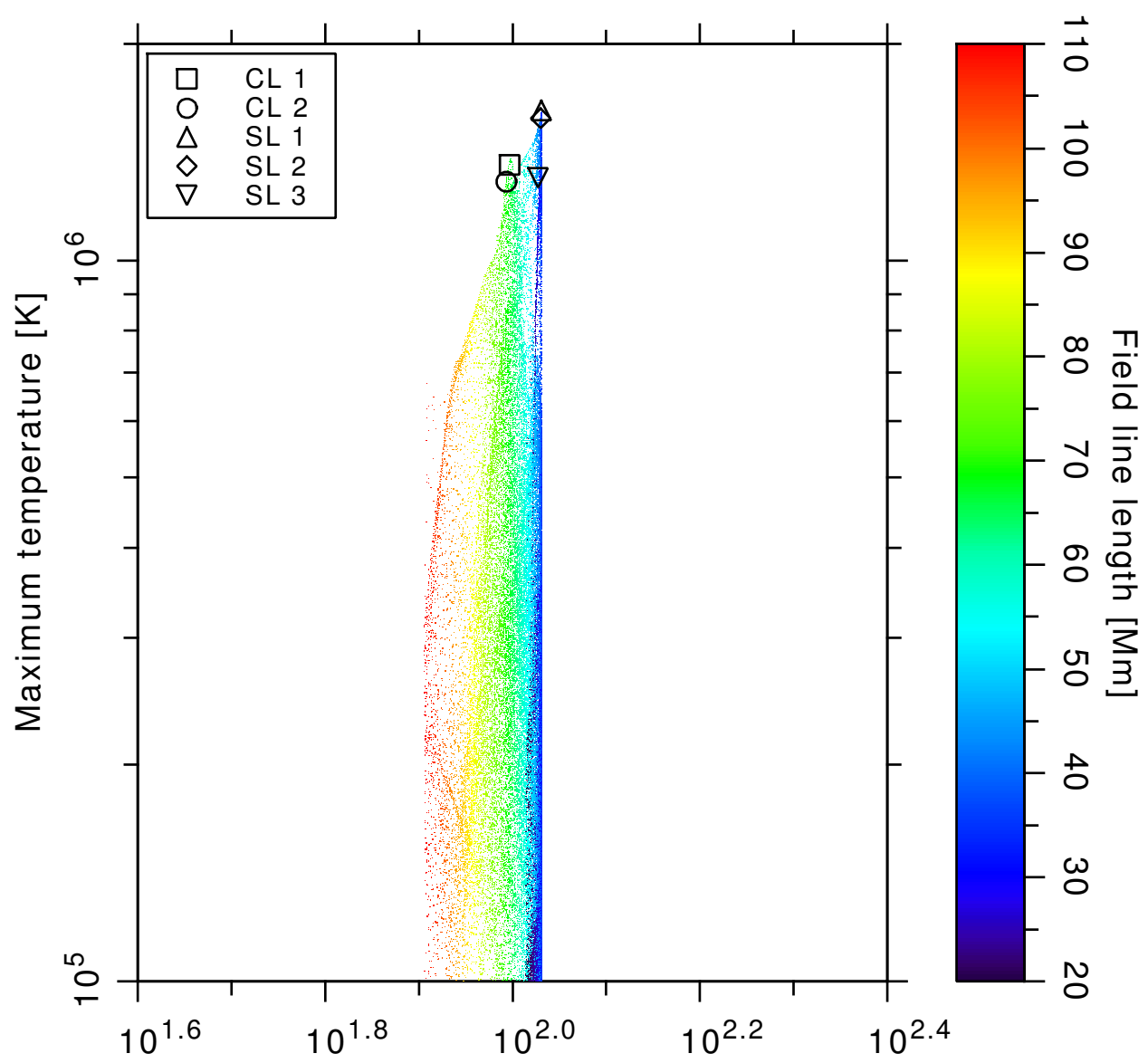

Length relation with Serio correction factor $\left(\mathrm{L}^{2 / 7} \mathrm{E}_{\mathrm{T}}^{5 / 7}\right)$

Figure A.3: Serio-RTV temperature (length and correction factor relation) versus maximum temperature along a field line.

is not much more than the length relation alone, but acting in the opposite direction, as is visible from the opposite ordering of the color code. 


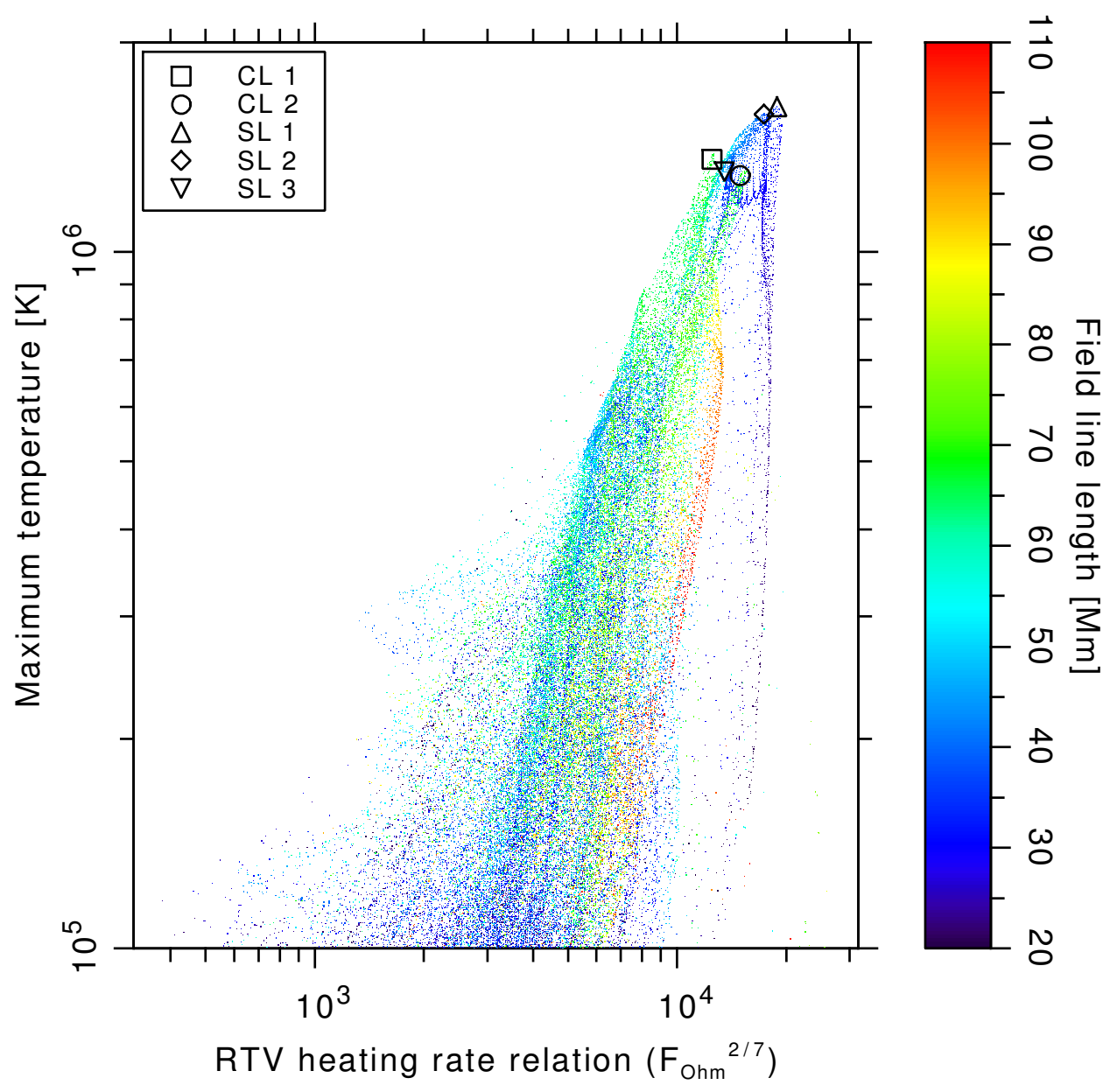

Figure A.4: RTV temperature (Ohmic heating relation) versus maximum temperature along a field line.

Finally, we find in Fig. A.4 that the overall shape of the whole Serio and RTV scaling laws are determined by the Ohmic heating relation $F_{O h m}^{2 / 7}$. Though, this doesn't mean that the length relation or the correction factor are irrelevant. But, in comparison of the Ohmic heating relation shown here and the full Serio scaling law in Fig. 4.12 we find that the length relation and the correction factor narrows the distribution of predicted loop temperatures, while the slope of the distribution of strongly heated field lines is mainly given by the Ohmic heating relation. 


\section{Publications}

* Bourdin, Ph.-A., Bingert, S., Peter, H., 2014, Coronal loops above an Active Region - observation versus model, PASJ, submitted

* Bourdin, Ph.-A., Bingert, S., Peter, H., 2013, Observationally driven 3D MHD model of the solar corona above an active region, A\&A, 555, A123

* Bourdin, Ph.-A., 2011, Denoising observational data, Contributions of the Astronomical Observatory Skalnate Pleso, 41, 149-155

* Up-to-date list of publications available online: http://www.Bourdin.ch/Philippe/Publications 



\section{Acknowledgements - Danksagung}

Special thanks go to my supervisor Hardi Peter for this trust in me and all the invested patience. In the same way I want to thank Sven Bingert, Tayeb Aiouaz, and my office mate Tijmen van Wettum.

Furthermore, I'm thankful for discussions with and support from (in high entropy order): Yana Maneva, Peter Caligari, Helmut Michels, Axel Brandenburg, Anders Johansen, Oskar von der Lühe, Oliver Wiloth, Rolf Schlichenmaier, Nazareth Bello González, Markus Roth, Rainer Hammer, Antonio Ferriz Mas, Jim Klimchuk, Luca Teriaca, Ines Dominitzki, Bernd Inhester, Jean Carlo Santos, Lukas Arnold, Fatima Rubio da Costa, Sofiane Bourouaine, Jens Niemeyer, Wolfgang Glatzel, Eric Priest, the people not listed here, and last but not least Yasuhito Narita.

I thank Suguru Kamio for his help finding active region observations, as well as Li Feng and Navdeep Kaur Panesar for introducing me to the stereoscopic 3D reconstruction, and Neda Dadashi for her suggestions regarding the absolute calibration of Doppler shifts. I cordially thank Marcela Bodnárová for proofreading.

Besonderer Dank gebührt meiner Mutter Siegrid Bourdin für Ihr Verständnis, dass das Lernen in eines Wissenschaftlers Leben niemals endet, meinem Vater Jean-Marie Bourdin für seine Hinweise bezüglich Design und Layout sowie meiner Schwester Joëlle-Beatrice Bourdin für ihre generelle Unterstützung.

Des Weiteren danke ich Svenja-N. Fischer für ihr geduldiges Warten auf Post, Marianne Junge für die cinéastischen Abende, Dennis Heese für seinen Aikido-Unterricht und meiner treuen Trainingspartnerin Catrin Sakel.

I also thank the kind reader for not giving up until now... 
Explicitly I want to thank the International Max-Planck Research School (IMPRS) on Solar System Physics, in particular its director Sami Solanki and its head Dieter Schmitt, as well as the Kiepenheuer-Institut für Sonnenphysik in Freiburg for the funding they provided.

The publication coming out of this work was partially funded by the Max-Planck-Princeton Center for Plasma Physics (MPPC).

The results of this research have been achieved using the PRACE Research Infrastructure resource Curie based in France at TGCC, as well as JuRoPA hosted by the Jülich Supercomputing Centre in Germany.

Preparatory work was executed at the Kiepenheuer-Institut für Sonnenphysik in Freiburg, as well as on the bwGRiD facility located at the Universität Freiburg, Germany.

Hinode is a Japanese mission developed, launched, and operated by ISAS/JAXA, in partnership with NAOJ, NASA, and STFC (UK). Additional operational support is provided by ESA and NSC (Norway).

The STEREO/SECCHI data used here were produced by an international consortium of the Naval Research Laboratory (USA), Lockheed Martin Solar and Astrophysics Lab (USA), NASA Goddard Space Flight Center (USA), Rutherford Appleton Laboratory (UK), University of Birmingham (UK), Max-Planck-Institut für Sonnensystemforschung (Germany), Centre Spatiale de Liège (Belgium), Institut d'Optique Théorique et Appliquée (France), and Institut d'Astrophysique Spatiale (France).

Finally, I thank all my former employers in and around Zürich and Freiburg for giving me the possibility to finance my dreams.

$\sim$ Vielen Dank $\sim$ Thank you $\sim$ Köszönöm Merci beaucoup 


\section{Curriculum Vitae}

Philippe Bourdin; Häuserstr. 2; D-37154 Northeim

Nationality: $\mathrm{D}+\mathrm{CH}$; marital status: unmarried Born on 14th of June 1975 in Friedberg/Hessen (Germany) http://www.Bourdin.ch/Philippe/

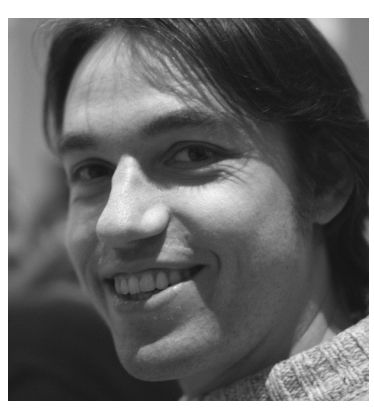

\section{Education and training / work experience:}

09/2013: Defense of PhD thesis (for Dr. rer. nat., "magna cum laude"), Göttingen/Germany

11/2011: PRACE Tier-1 Workshop on High Performance Computing, Amsterdam/Netherlands

09/2010: Single Dish School in the Era of Arrays, radio telescope Effelsberg, Bonn/Germany

01/2010-09/2013: IMPRS PhD-stipend; Editorial post-refereeing assistant, A\&A, supervisor: Hardi Peter

11/2009: 10-days observational campaign on VTT, Tenerife/Spain

10/2009: Solaire-Network school on project management and team work, Tenerife/Spain

10/2009: 2-days research stay, Institute for Advanced Simulation, Jülich/Germany

06/2009: USO summer school on solar magnetism, Dwingeloo

04/2009: Spring conference of the DPG, astronomy and plasma physics division, Greifswald

03/2009: $39^{\text {th }}$ Saas-Fee advanced course on magnetic fields of stars, Les Diablerets

11/2008: Solaire-Network school on Data Visualization at NBI, Copenhagen

09/2008: Participation in the LOC of the ESPM 12 in Freiburg

06/2008-09/2013: PhD-student in the coronal dynamics group, supervisor: Hardi Peter (KIS and MPS)

05/2008: Scientific assistant, Monte-Carlo simulations of light-scattering in optically thick media (human skin, "livor mortis" project), Freiburg Materials Research Center (FMF)

02/2007-04/2008: Main practical (until 04/2007) and Diploma thesis: "Implementation of a chargetransfer model and application to amorphous $\mathrm{Al}_{2} \mathrm{O}_{3}$ and corundum", Fraunhofer Institute for Mechanics of Materials (IWM)

01/2007: Scientific assistant, Software development for the "Brain-Machine-Interface" project, Bernstein Center for Computational Neuroscience (BCCN), Freiburg

12/2006: Main exams for the Diploma degree

02/2004-03/2007: Scientific assistant, Non-linear regularisation of experimental data with new theoretical models for parameter estimation in rheology / Monte-Carlo simulations of lightscattering in optically thick media (polymer blends) with the Mie-theory, (FMF)

04/2003-03/2006: 8 semester of physics, astronomy and computer science at the University of Freiburg

01/2000-03/2003: Company foundation and operation of "PAB Software \& Design" in Zürich

02/1997-01/2000: Diverse employments and contracts as webmaster, database designer and software engineer (Java, C/C++, Perl, PHP, SQL, Unix-shell). References: Institute for information retrieval/ETH Zürich, eurospider, CableCom, SwissRates, Unitek, UBS, Starseed, Furrer \& Partner, Pixelpark, Winthertur Versicherungen

10/1996-03/2003: Civil protection service of the city of Zürich, apprenticeship for medical attendance assistant and patient care, sporadic mandatory services in a geriatric clinic in Zürich

10/1996-03/2001: 5 semester of part time studies in physics, mathematics and music at the ETH Zürich

03/1996: Entrance examination at ETH Zürich (physics mark: 6/6)

09/1995-08/1996: Position as socio-paedagogical tutor at the Deutscher Kinderschutzbund, Friedberg

10/1995-09/1996: Selected courses in mineralogy at the University of Gießen

09/1994: Loan on the exhibition "Prometheus": a virtual roboter, based on artificial intelligence

07/1994-06/1995: Military service as a Medical Corps soldier in the Deutsche Bundeswehr

07/1985-06/1994: Augustinerschule Friedberg (Gymnasium) and graduation (Abitur) 


\section{Publications / Presentations / Conferences / Posters:}

(T) 05/2014: EGU General Assembly 2014, Vienna/Austria

(T) 04/2014: $4^{\text {th }}$ Internat. Workshop on small scale Solar Magnetic Fields, Bairisch Kölldorf/Austria

(A) 03/2014: "Coronal loops above an Active Region - observation vs. model" (PASJ, submitted)

(T) 02/2014: Colloquium, Kiepenheuer-Institut für Sonnenphysik, Freiburg/Germany

(T) 11/2013: Astrophysical Seminar, Kyoto University, Kyoto/Japan

(T) 11/2013: Hinode-7, Takayama/Japan

(T) 10/2013: $2^{\text {nd }}$ STORM Turbulence Workshop, Graz/Austria

(T) 06/2013: Pencil Code User Meeting 2013, Lund/Sweden

(T) 03/2013: 2013 LWS / SDO Science Workshop, Cambridge (MD)/USA

(A) 01/2013: "Observationally driven 3D MHD model of an Active Region" (A\&A, 2013, 555, A123)

(T) 10/2012: Rocks'n'Stars conference, Göttingen/Germany

(T) 08/2012: Hinode-6 meeting, St. Andrews/Scotland/UK

(T) 06/2012: Pencil Code User Meeting 2012, Helsinki/Finland

(P) 02/2012: $\quad$ th $^{\text {th }}$ NIC Symposium 2012, Jülich Supercomputing Centre, Jülich/Germany

(P) 01/2012: $511^{\text {th }}$ WE-Heraeus-Sem. "From the Heliosphere into the Sun", Bad Honnef/Germany (best poster award)

(CP) 10/2011: "Denoising observational data" (CAOSP 41, 2)

(P) 09/2011: $13^{\text {th }}$ European Solar Physics Meeting (ESPM), Rhodes/Greece

(T) 02/2011: $71^{\text {th }}$ DGG meeting with AEF workshop on numerical methods, Köln/Germany

(P) 05/2011: $4^{\text {th }}$ Solaire-Network meeting, Teistungen/Germany

(P) 10/2010: Hinode-4 meeting, Palermo/Italy

(T+P) 10/2010: $\quad$ EAST workshop on Solar Physics, Tatranska Lomnica/Slovakia

(P) 11/2009: $\quad 3^{\text {rd }}$ Solaire-Network meeting, Tenerife/Spain

(T) 10/2009: Colloquium at Institute for Advanced Simulation, Jülich/Germany

(T) 08/2009: Pencil Code User Meeting 2009, Heidelberg/Germany

(T) 04/2009: $5^{\text {th }}$ Black Forest Grid Workshop, Freiburg/Germany

(P) 04/2009: Spring conference of the DPG, astronomy and plasma physics division, Greifswald/G.

(P) 09/2008: $12^{\text {th }}$ European Solar Physics Meeting (ESPM), Freiburg/Germany

A: refereed journal Article, T: Talk, P: Poster, CP: refereed Conference Proceedings

\section{Computing time grants:}

05/2013-10/2014: 05/2012-04/2013: 11/2011-10/2012: 05/2011-10/2012: 05/2011-04/2012: 03/2011-07/2011: 05/2010-04/2011: 11/2009-08/2010: 06/2008-12/2009:
Curie, x86 Intel Nehalem, TGCC/France, CPU-hours: 5'940'000 ( $290 \mathrm{k} €)$ JuRoPA, x86 Intel Nehalem, NIC/Germany, CPU-hours: 1'248'000 ( $60 \mathrm{k} €)$ SuperMUC, x86 Intel Nehalem, LRZ/Germany, CPU-hours: 700'000 ( $35 \mathrm{k} €$ ) Curie, x86 Intel Nehalem, TGCC/France, CPU-hours: 6'600'000 ( $320 \mathrm{k€)}$ JuRoPA, x86 Intel Nehalem, NIC/Germany, CPU-hours: 2'880'000 ( $140 \mathrm{k} €$ ) Curie, x86 Intel Nehalem, TGCC/France, CPU-hours: 200'000 ( $10 \mathrm{k€})$ JuRoPA, x86 Intel Nehalem, NIC/Germany, CPU-hours: 1'056'000 ( $50 \mathrm{k€)}$ JuRoPA, x86 Intel Nehalem, NIC/Germany, CPU-hours: 96 '000 ( $5 \mathrm{k} €)$ bwGRiD, x86 AMD Opteron, University of Freiburg/Germany 